\title{
Sintering Stress and Microstructure in Ceramic Powder Compacts
}

May-Ying Chu

(Ph. D. Thesis)
Department of Materials Science and Mineral Engineering
University of California at Berkeley
and

Center for Advanced Materials

Materials and Chemical Science Division

Lawrence Berkeley Laboratory

University of California

Berkeley, CA 94720

August, 1990

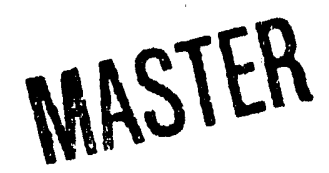

This work was supported by the division of Materials Science, Office of Basic Energy Research, United States Department of Energy, under contract No. DE-AC0376SF00098 with the Lawrence Berkeley Laboratory.

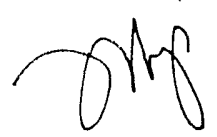




\title{
Sintering Stress and Microstructure in Ceramic Powder Compacts
}

by

\author{
May-Ying Chu
}

\begin{abstract}
The behavior of the sintering stress and microstructure during sintering is studied under various thermal and physical conditions. Specifically, the study includes sintering under isothermal or constant heating rate conditions; and altering the starting compact structure by pre-coarsening to increase the particles size, or by compaction to increase the starting density. Loading dilatometry is used to measure the ratio of the densification strain rate to the creep strain rate and the continuous shrinkage history of the systems. The data show that the ratio of the densification to the creep strain rate is remarkably constant within a wide temperature range for densification, from the earliest stages of densification to at least the onset of the final stage. As a consequence, the sintering stress, which is proportional to the strain rate ratio, is nearly independent of sintered density or of temperature. Analysis leads to a simple method for determining the simultaneous densification strain rate over the creep strain rate ratio.

A rigorous densification strain rate equation is derived for a model system. The constant sintering stress allows progress on formulating a sintering equation to predict the densification behavior of powder compacts. Analysis of dilatometry data from compacts sintered at various heating rates, and of numerical computations, indicate the need to include separate coarsening processes with different activation energies. The same model is used to describe the differences in pore spacing evolution between pre-coarsened and as-received systems.
\end{abstract}




\section{Acknowledgements}

I would like to thank the many people who have assisted in the completion of this work. In particular:

Lutgard De Jonghe, my advisor, for giving guidance throughout my stay. I especially valued the many conversations we had, and the editorial assistance during the writing of my thesis.

Len Rahaman and Mark Lin, for helping me with the dilatometer.

Professors Alan Searcy and David Bogy for reading this thesis.

All the members of my research group, especially Thea Buchanan, for their patience and good humor.

Charles Alcock, my husband, for always being there with his support and encouragement. 


\section{Table of Contents}

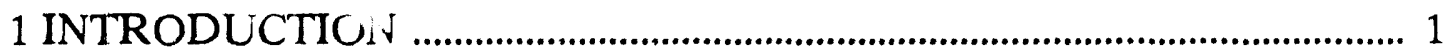

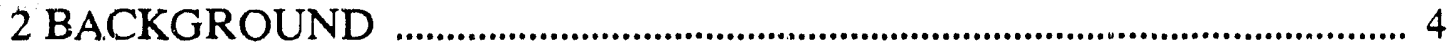

2.1 General Description ............................................................................. 4

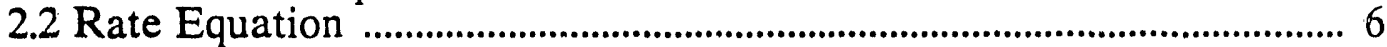

2.2.1 Driving Force ....................................................................................... 6

2.2.2 Densification Equations ................................................................... 10

2.2.3 Creep Equations ......................................................................... 11

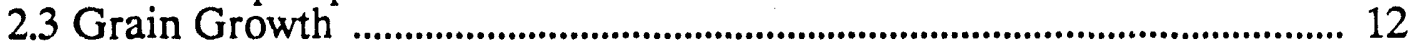

3 EFFECT OF TEMPERATURE ON DENSIFICATION AND CREEP

STRAIN RATES DURING SINTERING …................................................... 15

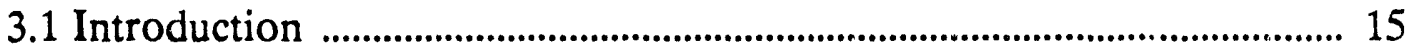

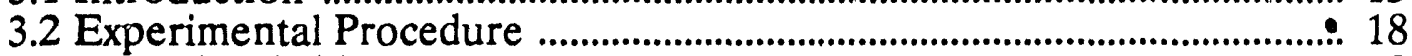

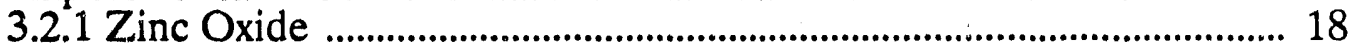

3.2.2 Aluminum Oxide .................................................................................. 19

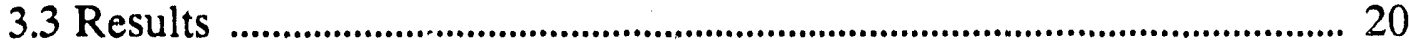

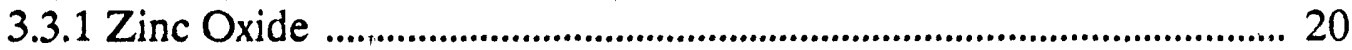

3.3.2 Aluminum Oxide ........................................................................ 21

3.4 Analysis ......................................................................................................... 21

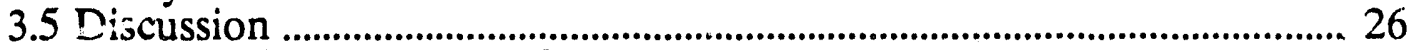

3.5.1 Densification Strain Rate .............................................................. 26

3.5.2 Model derivation of Strain Rate Equation ................................... 28

3.5.2.1 Grain Boundary Diffusion .......................................................... 29

3.5.2.2 Volume Diffusion ................................................................... 32

3.5.2.3 General Isothermal Densification Strain Rate Equation ..... 32

3.5.2.4 Creep Strain Rate .................................................................... 33

3.5.2.5 Ratio of Densification Strain Rate / Creep Strain Rate ...... 33

3.5.3 Densification Viscosity / Creep Viscosity ........................................ 34

3.6 Summary .............................................................................................. 36

4 EFFECT OF HEATING RATE ON SINTERING AND COARSENING

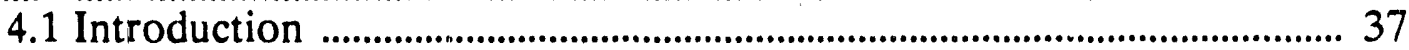

4.2 Experimental Procedure ............................................................................. 41

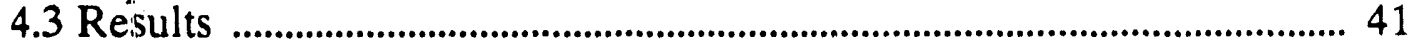

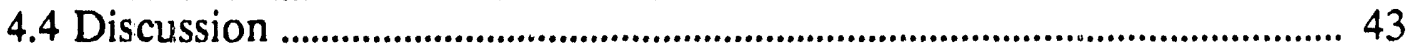

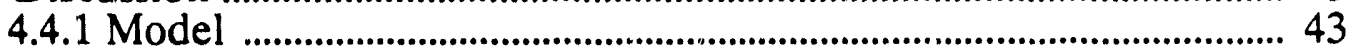

4.4.2 Limiting Cases of No Coarsening and Extreme Coarsening .......... 50

4.4.3 Numerical Calculations of the Coarsening Function, $x^{m}$............... 54

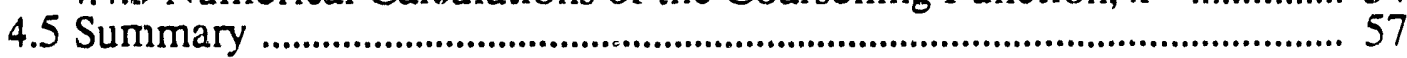


5 EFFECT OF COARSENING ON SINTERING AND

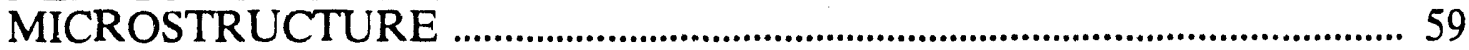

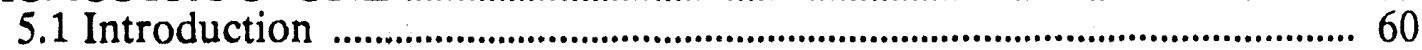

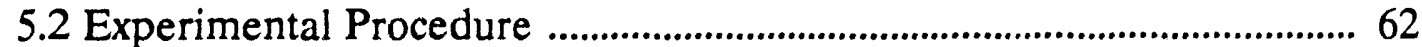

5.2.1 Magnesium Oxide ...................................................................................... 62

5.2.2 Aluminum Oxide ......................................................................................... 63

5.2.3 Zinc Oxide .................................................................................................. 63

5.3 Results .................................................................................................... 64

5.3.1 Sintering of Magnesium Oxide and Aluminum Oxide ....................... 64

5.3.2 As-received versus Pre-coarsened Zinc Oxide ..................................... 64

5.4 Discussion .......................................................................................................... 67

5.4.1 Effect of Pre-coarsening on the Sintering Stress ............................... 67

5.4.2 Effect of Pre-coarsening on the Evolution of the

Center-to-center Pore Spacing, $x(t, T)$......................................................... 69

5.4.3 Modeling of the Evolution of the Interpore Spacing, $\mathrm{x}(\mathrm{t}, \mathrm{T})$......... 71

5.4.4 Application of Pre-coarsening and Two-step Sintering .................. 73

5.5 Summary ……................................................................................................. 75

REFERENCES ……........................................................................................... 77

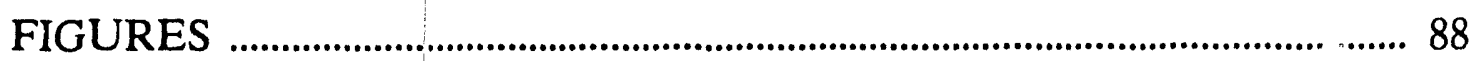




\section{INTRODUCTION}

Sintering is the process by which a porous powder compact transforms into a denser material with the application of heat. This procedure has been used for thousands of years beginning with the formation of clay ware, and generally consists of two stages. In the formation stage loose powders are molded into the desired shape, and then in the firing stage the powder particles in the body fuse together to form a denser solid body. Although the process of sintering has been used for so long the theory and the modeling of the process did not begin until the mid-1940's. This lack of interest in part was due to the fact that the applications of the original clay products were not very demanding. However, since ceramics are good heat and corrosion resistant materials their application grew. The need for greater control over the production process has motivated recent interest in understanding the sintering process.

Many theories of sintering have been devised in the past fifty years. Several review articles give good summaries of the field [Burke and Rosolowski 1976, Coble and Cannon 1978, Exner and Petzow 1980, German 1984, Kuczynski 1985].

One of the first rigorous attempts to develop a theory on sintering was by Frenkel [1945]. He assumed crystalline bodies can approach each other through viscous flow under the influence of their surface tensions, and derived an equation for the coalescence of two liquid particles. Later in 1949 Kuczynski did a series of experiments measuring the neck growth rates between metal spheres on metallic blocks. By sintering the spheres on blocks at different temperatures for various times, and then polishing and examining the cross-section to measure the local geometry, he identified the mechanism for sintering. 
Herring in 1950 derived the "scaling laws" which identify various mechanisms for sintering. Under the assumption that s:ze is the only variable between two systems during isothermal sintering, by measuring the amount of time necessary for the two monosize powder systems to enlarge themseives by the same ratio the mechanism in principle can be determined. In practice this proves to be difficult, since for different size powders contamination and orientation may vary significantly [Song and Coble 1984].

Many other works followed which tried to identify the various mechanisms and/or activation energies of sintering. Models of two particle systems with specific geometry such as spheres, cylinders, and plates were developed [Kuczynski 1949, Kingery and Berg 1955, Alexander and Balluffi 1957, Coble 1958 \& 1961b, Nichols 1966, Johnson 1969]. These studies were mainly interested in identifying the active mechanism for sintering. Other models were based on multiparticle arrangements [Exner and Petzow 1976, Hsueh and De Jonghe 1984], arrays of particles [Exner et al 1973, Fxner and Petzow 1976, Exner 1979, Lange and Kellet 1986], and numerical methods [Kuczynski 1976, Bross and Exner 1979, Hwang and German 1984].

As is evident from the vast number of papers published, a model which can predict the sintering behavior of a ceramic does not yet exist. The basic problem in modeling is that although great accuracy can be attained by restricting the number of parameters, it is difficult to describe a real compact which has many more variables. Some investigators attempt to determine the mechanism for sintering by comparing data to a known model. However, in a given experiment it is difficult to isolate a single mechanism, and to measure and quantify the local geometry and parameters (e.g. particle/pore sizes and curvatures, dihedral angle, surface and grain boundary energies). It has been shown that frequently many models can fit the data equally well 
[Exner and Petzow 1980, Johnson 1982].

The objective of the present study is to bypass all the local geometrical parameters needed to calculate the driving force and to replace it with a global parameter, $\Sigma$, the sintering stress. This stress, defined as the equivalent external mechanical stress that would give rise to the same densification strain rate as the internal driving force, can be obtained by simultaneous creep and densification experiments. Previous experiments performed under isothermal conditions have indicated $\Sigma$ remains constant throughout a wide density range [Chu et al 1989]. This study, by using constant heating rates, examines the influence of temperature on $\Sigma$ and its behavior in the initial stage. An additional objective is to develop a simple densification rate equation that will be useful in both interpreting and controlling sintering.

For clarity the thesis has been divided into four additional chapters. Chapter 2 will describe general sintering terms, observations, and theories that are not specifically mentioned in the later chapters. The remaining three chapters will describe and discuss individual topics. First, the effect of temperature on the densification and creep strain rate will be studied in chapter 3 . Here, constant heating rate sintering conditions are used and the results are compared to data obtained under isothermal conditions. A rigorous densification strain rate equation is derited for a model system and its implications for the sintering stress are discussed. Next, in chapter 4 the effect of heating rate on sintering and coarsening will be discussed. A coarsening equation including two classes of processes, those associated with densifying and with non-densifying mechanisms, is introduced. Dilatometry data and numerical calculations show the utility of the model. Finally, in chapter 5 the effect of coarsening on sintering and microstructure is studied. The same coarsening model is used to interpret differences in the microstructure between the coarsened and the as-received system. 


\section{BACKGROUND}

This chapter has been divided into three main subsections. First, the common descriptions used in sintering are presented. Next, a discussion of rate equations will follow; for clarity the driving force terms will be discussed separately. Finally, since a critical issue in sintering is coarsening, i. e. the size increase in grains and/or pores, grain growth and its relationship to pore spacing will be discussed.

\subsection{General Description}

Sintering theories are usually divided into three stages: 1) initial stage, where the particles form neck contacts with each other, 2) intermediate stage, where the compact forms an open network of pores, and 3) final stage, where the pores are isolated from each other [Coble 1961, Johnson and Cutler 1963, Johnson 1970, Eadie et al 1974 \& 1978, Handwerker et al 1984].

The initial stage of sintering involving neck formation only accounts for the first several percent of shrinkage. During the intermediate stage of sintering the pores are interconnected. Here the density of the material increases dramatically, but sintering is complicated by the additional process of grain growth. The final stage of sintering begins when the channel network of pores begin to break off and the pores become isolated from each other. This usually begins when the compact density reaches above about 0.9 of its theoretical density. In this last stage the densification rate decreases rapidly.

Although the stages of sintering are clearly distinguished, in a real compact where regions have different packing densities the separation of the stages is less clear since the various stages of sintering can occur simultaneously [Barrett and Yust 1967, Weiser and De Jonghe 1986] 
The driving force (see section 2.2.1) for sintering comes from the reduction of the free energy of the system by the replacement of solid-vapor interfaces with solid-solid interfaces. The particle neck curvature generates a stress on the grain boundary, which produces a chemical potential gradient that causes material to flow from the grain boundary to the neck.

The transport mechanisms of sintering can be separated into two categories, densifying and non-densifying. Viscous flow, grain boundary diffusion, and volume diffusion are all processes which will cause an overall decrease in the volume of the sample or, specifically for the two particle system, the center-to-center approach. Non-densifying mechanisms include evaporation/ condensation and surface diffusion. Although these processes do not shrink the sample ihey do change the microstructure, which in turn will affect subsequent densification. The various paths for these mechanisms in a two particle system are shown in Fig. 2.1. Sintering diagrams have been developed to identify the dominant mechanism at a given temperature and neck geometry [Ashby 1974, Swinkels and Ashby 1981]. In principle, the sintering microstructure can be manipulated by picking the appropriate heat treatments; however, in practice the utility of these maps prove limited since they are derived from models based on very restricted geometries.

It is commonly observed during isothermal sintering that the compact densifies more slowly with increasing sintering time. Empirically, the sintered density is found to be approximately proportional to the sintering time to some power (power law densification), e. g. Burke [1962]:

$\Delta \rho \propto t^{q}$

Eqn. 2.1 
where $\Delta \rho$ is the instantaneous density increase, $t$ is the time, and $q$ is a fraction between $1 / 2-1 / 3$. This density increase has also been descrived as logarithmic densification, e. g. Vieira and Brook [1984]:

$$
\Delta \rho \propto \ln (t)
$$

Eqn. 2.2

Microstructural studies indicate that during sintering the density increase of the compact is almost always accompanied by increases in the grain and pore sizes. At the high densities, during the final stage, pores may break away from the grain boundaries; these trapped pores are seldom removed and prevent the compact from reaching its fully dense state.

\subsection{Rate Equation}

The rates for all processes will depend on both a driving force term and a kinetic term. The study of sintering rates can be separated accordingly. This section will be divided into three parts. First, work on the driving force for sintering will be described. Next, the common form used for sintering rate equation will be presented. Finally, since much of this study depends on the simultaneous process of creep and densification, creep rate equations will be described.

\subsubsection{Driving Force}

The sintering driving force comes from the reduction of the free energy of the system by the replacement of solid-vapor interfaces with solid-solid interfaces. The neck curvature causes a stress on the grain boundary, this in turn produces a chemical potential gradient which causes material to flow from the grain boundary to the neck. Alternatively, the grain boundary can be considered as the sink for the vacancies 
supplied at the neck (Fig. 2.2). In actuality the driving force can depend on many variables, including the surface free energy, grain boundary energy, the dihedral angle, pore/particle curvatures and distributions, and coordination number [Johnson 1969, Exner and Bross 1979, Cannon 1981., Searcy 1985, De Jonghe et al 1989, Kellet and Lange 1989, Cannon and Carter 1989]. The difficulty in applying these driving force models is the need of local geometry which even in very uniform systems is hard to measure. In a real compact, since the material is very inhomogeneous, it is impossible to obtain all of the necessary information.

In the simplest case one can examine a spherical isotropic pore in a body; associated with the surface of the pore is a compressive stress described by the Kelvin equation

$$
\sigma_{c}=\frac{2 \gamma_{s}}{r}
$$

where $\sigma_{c}$ is the stress on the surface, $\gamma_{s}$ is the surface free energy, and $r$ is the radius of the pore. This predicts that smaller pores generate a higher compressive stress. This compressive stress can be thought of as an equivalent externally applied hydrostatic pressure which will cause the material to shrink (Fig. 2.3). The earlier work on sintering used $\sigma_{c}$ as the driving force [Frenkel 1945, Mackenzie and Shuttleworth 1949, Coble 1961a]

Strictly, the driving force for densification would depend both on the particle radius and the neck radius [Cannon 1981, Raj 1987, De Jonghe and Rahaman 1988], so that 


$$
\sigma_{c} \propto \frac{Y_{s}}{r} \therefore \frac{Y_{b}}{G}
$$

where $Y_{b}$ is the free energy of the boundary and $G$ is the radius of the grain. In a multiparticle compact the basic relationshıp still holds; however, the analysis is vastly complicated by the numerous curvatures (positive and negative) present in the system. Additionally, the detailed structural geometry will depend on many factors, such as $\gamma_{b}$ ,$Y_{s}$, dihedral angle, and pore coordination number, which are not readily accessible. Experimentally, the necessary values for computing the driving force are at best tedious and at worst impossible to quantify. An epoxy replica of $\mathrm{ZnO}$ pore structure made by pressure infiltration is shown in Fig. 2.4. As is evident from the micrograph the pure network is very complicated.

The role of the microstructure, and its effects on sintering of powder compacts have been studied by many [Rhines et al 1950, DeHoff et al 1966, Whittemore and Varela 1980, DeHoff 1984, Rhines and DeHoff 1984]. In general the investigators concentrated on the structural evolution of the pore network. Gregg and Rhines [1973] attempted to measure the driving force for copper compacts from zero-strain rate conditions obtained during tensile loading of sintering specimens. In the systems used, the driving force increased with increasing density. This increase was correlated with the increasing curvature of the pores as they shrank [Gregg and Rhines 19;3, Aigeltinger 1975]. In another study by Venkatachari and Raj [1986] the driving force decreased with increasing density. This result was attributed to the decrease in por? curvature from pore coalescence.

More recently, De Jonghe and Rahaman used a different, and perhaps simpler, experimental technique known as loading dilatometry [De Jonghe and Kahamän 
1984a, Rahaman and De Jonghe 1984, Rahaman et al 1986] to determine the driving force. Simultaneous measurements of creep and densification, under conditions of low applied uniaxial loads, led to an interesting observation on many ceramic powder systems studied: the ratio of the densification strain rate to the creep strain rate is constant throughout large variations in density. This result gives strong indications that the sintering stress, $\Sigma^{*}$, which is believed to be proportional to this ratio, does not vary during densification (for a more detailed discussion see section 3.5). A feature of their studies is that the hydrostatic component of the applied uniaxial stress is low compared to the sintering stress produced by the system. Consequently, the densification mechanism for the loaded and the free sintered samples is equivalent. Subsequent work by Venkatachari and Raj [1986] used much higher stresses to investigate creep and densification, their work would therefore be more appropriate to sinter-forging.

Although numerous loading dilatometry studies show that the strain rate ratio is constant throughout the measured density range, the value of this ratio is system specific (see Fig. 3.1). For sintering compacts with the same starting particle size this ratio only changes with variations in starting density. The compact with the higher starting density has a higher $\Sigma$. This result is consistent with Eqn. 2.4, since comparison is made at constant density and the compact with the higher starting density will at some tixed time have a smaller grain size. This implies that $\Sigma$ can be changed by forcing a system through a different microstructural path, which can be accomplished by :ntentional coarsening. The microstructure of the system can also be changed by heat

\footnotetext{
- $\Sigma$ is defined as the equivalent external mechanical stress that is necessary to give rise to the same densification strain rate as the internal driving force of the system (see Fig. 2.5). $\Sigma$ is also called the sintering force [DeHoff 1966], the sintering potential [Hsueh et al 1986a], or the sintering pressure [Raj and Bordia 1984].
} 
treating the compact under different atmospheres [Lee and Readey 1984, Quadir and Readey 1984, Readey et al 1984] or at different temperatures (see chapter 5). The effect of temperature and coarsening on the sintering stress is investigated in chapters 3 and 5 , respectively.

\subsubsection{Densification Equations}

Evaluation of the sintering stress will contribute to reaching the final goal, to obtain an equation that can describe and predict the sintering behavior of the system. Since the sintering stress, $\Sigma$, does not change much during the course of sintering, the assumption can be made that the driving force is independent of the density. This will make subsequent modeling of the sintering equation much simpler.

The sintering rate equation is written in the following generic form by many investigators [Frenkel 1945, Mackenzie and Shuttleworth 1949, Coble 1961a\&b and 1970, Johnson 1969, Beere 1975c, De Jonghe and Rahaman 1984b \& 1988, Scherer 1984, De Jonghe et al 1986, Evans and Hsueh 1986, Hsueh et al 1986a\&b, Rahaman et al 1986].

$$
\dot{\epsilon}_{d}=\frac{1}{\eta_{d}}\left(\Sigma+\sigma_{a}\right) \quad \text { Eqn. } 2.5
$$

where $\eta$ contains the various kinetic parameters such as the diffusion coefficient, temperature, and diffusion length (see section 3.5.2), $\Sigma$ is the driving force supplied by the system, and $\sigma_{a}$ is the additional applied hydrostatic stress. 


\subsubsection{Creep Equations}

The creep rate equation upon uniaxial loading by a stress $\sigma$ is commonly written in a form similar to the sintering rate equation:

$$
\dot{\epsilon}_{c}=\frac{1}{\eta_{c}}(\sigma)^{s}
$$

where again $\eta_{c}$ contains the various kinetic and structural parameters, $\sigma$, the uniaxially applied stress, is the driving force $\left(\sigma=3 \sigma_{a}\right)$, and s, the stress exponent, is dependent on the creep mechanism ( $s=1$ for diffusion, $s=2$ for interface reactions, and $s \geq 3$ for dislocation movement [Brook 1982a]).

Under the present experimental conditions of low applied stress, creep occurs by solid state diffusion and s equals 1 [Johnson 1988]. The uniaxially applied load changes the concentration of vacancies at the grain boundaries. The associated vacancy gradient causes vacancies to migrate from boundaries in tension to boundaries in compression. The vacancies can diffuse either through the bulk or through the grain boundary. Nabarro [1947] and Herring [1950] modeled bulk diffusion and arrived at the general form

$$
\dot{\epsilon}_{c}=\frac{A D_{v} \sigma}{k T G^{2}}
$$

where $A$ is a constant, $D_{v}$ is the volume diffusion coefficient, $k$ is Boltzman's constant, $\mathrm{T}$ is the absolute temperature, and $\mathrm{G}$ is the grain size. Later in 1963 Coble modeled vacancy migration through the grain boundary and proposed 


$$
\dot{\epsilon}_{c}=\frac{A^{\prime} D_{b} \delta \sigma}{k T G^{3}}
$$

where $A^{\prime}$ is a constant, $D_{b}$ is the grain boundary coefficient, and $\delta$ is the grair boundary thickness.

\subsection{Grain Growth}

Densification is nearly always accompanied by grain growth. Even in the best monosize powder the accompanying grain growth is about a factor of two [Barringer et al 1984]. For most sintered systems the final grain size is commonly one or two orders of magnitude larger than the initial particle size. The grain growth rate in dense and porous system have been studied extensively; several gond reviews are by Hillert [1965], Nichols [1966], Brook [1976], and Atkinson [1988].

Grain growth is usually divided into two categories: normal, where the normalized grain size distribution remains constant with time, and abnormal or discontinuous, where a few large grains increase rapidly and the normalized distribution is not maintained. Only normal grain growth will be discussed here since it is more relevant to solid state sintering.

The driving force for normal grain growth is the reduction of interface energy" throughout the system. It is an activated process where the atoms migrate across grain boundaries from grains with concave boundaries into the neighboring grains. The migration is driven by the chemical potential gradient caused by curvatures differences between the grain boundaries.

- Crystals have anisotropic interface energies, but for the present discussion grains are assumed to have isotropic interface energies. 
Empirically, grain growth most often follows a power law of the form,

$$
G^{m} \propto t
$$

Eqn. 2.9

where $G$ is the grain size, $t$ is the time, and $m$, the grain growth exponent, is usually equal to 3 for porous system but can range from 1 to 4 [Brook 1976, Atkinson 1988].

For a dense single phase system a parabolic grain growth law, where $\mathrm{m}=2$, can be derived easily. The driving force for grain growth is the reduction of grain boundary energy throughout the entire system and should be proportional to the average curvature, which scales with the inverse of the grain size. The grain growth rate, $\dot{G}$, then is

$$
\dot{G} \propto \frac{K}{G}
$$

where $\mathrm{K}$ is a constant, and $G$ is the grain size. Upon integration

$$
G^{2}=G_{0}^{2}+K t
$$

since at $t=0$ the grain size must equal the starting grain size. Grain growth is an activated process, therefore Eqn. 2.11 is usually written as

$$
G^{2}=G_{0}^{2}+C_{0} \exp \left(\frac{-Q_{g}}{k T}\right) t
$$


where $C_{o}$ is a constant $Q_{g}$ is the activation energy for grain growth, $k$ is Boltzman's constant, and $\mathrm{T}$ is the absolute temperature.

Generally, the cubic "law", where $m$ is equal to 3 , is reported in both dense and porous systems [Coble 1961b, Greskovich and Lay 1972, Coleman and Beere 1975, Petrovic et al 1985]. This could indicate that the driving force does not equal $1 / \mathrm{G}$. Brook [1976] proposed that the exponent $m$ is determined by the dominant mechanisms for grain boundary migration. The growth rate is approximately equal to the average velocity of the grain boundaries, which is a product of the driving force and the motility of the grain boundary. The driving force still arises from the curvature $(1 / G)$, but the mobility can vary. If the mobility remains constant then parabolic behavior results. However, as growth proceeds, the boundary mobility may decrease due to increases in impurity segregation. Additionally in the porous systems, when pores travel with the boundaries, the boundary mobility will be affected by pore drag [Kingery and Francois 1965, Carpay 1977].

Coarsening is usually described in terms of the evolution of the grain size. For dense systems and porous systems with pores on each grain boundary junction this is valid. However, model-based descriptions of densification strain rates should be based on the mean interpore separation (see section 3.5.2). A convenient assumption is to put the grain size about equal to the pore spacing since grain size data are available; but a microstructural examination of most partly-sintered compacts reveals that the two spacings may be quite different (see section 4.4.1). 


\section{EFFECT OF TEMPERATURE ON DENSIFICATION AND CREEP STRAIN}

\section{RATES DURING SINTERING ${ }^{*}$}

\section{Abstract}

The densification and the creep strain rates of polycrystalline $\mathrm{ZnO}$ and of $\mathrm{Al}_{2} \mathrm{O}_{3}$ powder compacts have been measured simultaneously in a loading dilatometer, under constant heating rate and constant temperature conditions. The data show that the ratio of the densification to the creep strain rate is remarkably constant within a wide temperature range for densification, from the earliest stages of densification to at least the onset of the final stage. This implies that the sintering stress, which is proportional to the strain rate ratio, is nearly independent of temperature or of sintered density. The analysis leads to a simple method for determining the simultaneous densification over the creep strain rate ratio. The implications of the result for the processing of heterogeneous ceramics and composites are discussed.

\subsection{Introduction}

It is widely recognized that the densification behavior of powder compacts depends significantly on their uniformity, and efforts to achieve a high degree of uniformity in the initial structure of powder compacts have been very fruitful. In many if not most practical powders, however, agglomerate formation cannot be completely avoided. Further, in a number of important cases, such as the particulate composites, inhomogeneities are an essential part of the system. The presence of heterogeneities in the powder compacts lead to local, non-uniform densification rates which, in turn, produce stresses in the surrounding matrix that oppose densification. Unless these stresses are relieved by shear deformation (i.e. creep) they can lead to a significant reduction in densification rates and may exacerbate the development of deleterious

\footnotetext{
* This chapter has been published in part in Chu et al [1989].
} 
crack-like voids around inclusions [Evans 1982, Raj and Bordia 1984, De'Jonghe et al 1986, Hsueh et al 1986a\&b, Rahaman and De Jonghe 1987a\&c, Scherer 1987, De Jonghe and Rahaman 1988].

$I_{\mathrm{r}}$ addition, clustering of the dispersed phase will lead to the formation of extended skeletal segments of the inert particles, arresting macroscopic densification, as was discussed by Lange [1987]. These clustering effects, rather than the back-stresses generated by local densification rate mismatch, are likely to dominate the densification behavior at high inclusion content in particulate composites. If techniques such as slip casting and use of coated particles were employed that prevented the formation of percolation networks of the inert phase, then the back-stresses which result from local densification rate mismatch would remain as the most significant factor impeding free sintering of particulate composite powder compacts. It has been shown by De Jonghe and Rahaman [1988] and by Bordia and Scherer [1988] that the back-stresses which arise during the densification of particulate composite powders are simply viscous, i.e., proportional to the instantaneous densification strain rate of the matrix. This results leads to a matrix densification strain rate that is determined by the volume fraction of the dispersed inert phase and by the ratio of the creep viscosity to the densification viscosity:

$$
\dot{\epsilon}_{m}=\frac{\dot{\epsilon}_{m o}}{1+4\left(\frac{\eta_{c}}{\eta_{d}}\right)\left(\frac{f}{1-f}\right)}
$$

where the superscript dot represents differentiation with respect to time and $\dot{\epsilon}_{m}$ is the densification strain rate of the matrix of the composite, $f$ is the volume fraction of 
the hard inclusion, $\eta_{c}$ and $\eta_{d}$ are the creep and densification viscosities of the matrix, respectively, $\dot{\epsilon}_{m o}$ is the matrix densification strain rate at $f=0$, defined by $\dot{\epsilon}_{m o}=-\Sigma / \eta_{d}$, where $\Sigma$ is the sintering stress.

The magnitude of the ratio of the creep viscosity to the densification viscosity of the matrix is therefore an important parameter in the densification of particulate systems in which the inclusion phase is uniformly distributed, even though this ratio is unlikely to vary by orders of magnitude, as first postulated by Hsueh et al [1986a], and Raj and Bordia [1984].

In general the analysis of sintering is done at a constant temperature; but practically, densification cannot be carried out isothermally, and it is well known that significant densification usually occurs before the isothermal sintering condition has been reached. Alternatively, non-isothermal processing methods might be considered to affect the microstructural evolution of the powder compact. Thus, the dependence of the ratio of the densification strain rate to the creep strain rate on temperature needs to be examined for non-isothermal conditions. From the ratio of the densification strain rate to the creep strain rate, the ratio of the creep viscosity over the densification viscosity can be inferred, allowing an assessment of the possible effects of heating schedules on densification of particulate composites.

Rahaman and De Jonghe [1984j have used the technique of loading dilatometry to measure simultaneously the densification and creep strain rates in a number of ceramic powder systems [Rahaman and De Jonghe 1984 \& 1987, Rahaman et al 1986, 1987 \& 1988, Lin et al 1987, De Jonghe and Srikanth 1988]. Generally, for each system, the densification and creep strain rates were measured at a single, isothermal sintering temperature. A selection of the data is shown in Fig. 3.1, where the ratio of the linear densification strain rate to the creep strain rate is plotted as a function of relative 
density. Over a wide density range, covering at least the intermediate stage of sintering, the ratio of the densification over the creep strain rate is almost independent of density. The value of this ratio decreases with decreasing initial (green) density ind is system specific.

In a study on the creep-sintering of soda-lime glass powder compacts [Rahaman et al 1986] conducted at two closely separated temperatures $\left(580\right.$ and $\left.605^{\circ} \mathrm{C}\right)$, the data indicated that the ratio of the de. sification to the creep strain rate was nearly independent of temperature (Fig. 3.1). The objective of the present section is to examine the dependence of the densification and creep strain rates on temperature from experiments conducted over a wide temperature range for a constant heating rate schedule. Data were obtained for two different crystalline powders: $\mathrm{ZnO}$ and $\mathrm{Al}_{2} \mathrm{O}_{3}$. For comparison, data were also obtained on the isothermal creep-sintering of alumina.

\subsection{Experimental Procedure}

\subsubsection{Zinc Oxide}

$\mathrm{ZnO}^{*}$ powder compacts $(6 \mathrm{~mm}$ in diameter by $6 \mathrm{~mm})$ having an initial relative density of $0.44 \pm 0.01$ were made by uniaxial compaction in a tungsten carbide die at $\sim 10 \mathrm{MPa}$. The same powder was used by Rahaman and De Jonghe [1987] in earlier sintering studies.

The compacts were sintered in air in a loading dilatometer [De Jonghe and Rahaman 1984a], under uniaxial loads of 0 or $7 \mathrm{~N}$, at a constant heating rate of $4^{\circ} \mathrm{C} / \mathrm{min}$. The experiment was stopped at $1000^{\circ} \mathrm{C}$, where densification was nearly complete. The load of $7 \mathrm{~N}$, corresponding to an initial stress on the green compact of $0.2 \mathrm{MPa}$, was

* Reagent grade, Mallinckrodt Inc., Paris, Ky 
applied when the temperature had reached $500^{\circ} \mathrm{C}$ when shrinkage just commenced. The mass and dimensions of the samples were measured before and after sintering and the final densities were verified using Archimedes' method. In a separate set of experiments, sintering under the load of $7 \mathrm{~N}$ was terminated at temperatures between 500 and $1000{ }^{\circ} \mathrm{C}$, and the dimensions of these compacts were measured using a micrometer.

Other compacts from the same powder which were pressed to an initial relative density of $0.53 \pm 0.01$ were sintered under similar conditions with constant heating rates of $2^{\circ} \mathrm{C} / \mathrm{min}, 4^{\circ} \mathrm{C} / \mathrm{min}$, or $8^{\circ} \mathrm{C} / \mathrm{min}$.

\subsubsection{Aluminum Oxide}

Procedures similar to those for $\mathrm{ZnO}$ were used for the $\mathrm{Al}_{2} \mathrm{O}_{3}$ powder ${ }^{*}$. The powders were compacted in a die to an initial relative density of $0.52 \pm 0.01$. They were then sintered in air at constant heating rate, up to a maximum temperature of $1400^{\circ} \mathrm{C}$. The loads of 0 and $7 \mathrm{~N}$ were applied from $1000^{\circ} \mathrm{C}$ on, at which point densification could not yet be detected. In addition to constant heating rate experiments, samples were isothermally sintered at $1350^{\circ} \mathrm{C}$ in air, for 2 hours, under a load of $7 \mathrm{~N}$. These samples were inserted rapidly into the hot zone of the dilatometer and reached the sintering temperature in about 5-7 min. After the initial temperature transient the load was applied.

* Alcoa Chemicals, Pittsburg, PA. 


\section{$\underline{3.3 \text { Results }}$}

\subsubsection{Zinc Qxide}

Figure 3.2 shows the results for the axial true strain, $\epsilon_{z}{ }^{*}$, versus temperature for the $\mathrm{ZnO}$ samples sintered at a constant heating rate of $4^{\circ} \mathrm{C} / \mathrm{min}$ under zero applied stress and under an initial stress of $0.2 \mathrm{MPa}$. Each curve is the average of two runs under the same conditions and each is reproducible to $\pm 1 \%$. The results for the axial true strain, $\epsilon_{z}$, versus the radial strain, $\epsilon_{r}$, determined by terminating the sintering runs at temperatures between 500 and $1000^{\circ} \mathrm{C}$, are shown in Fig. 3.3; each data point was obtained using a different sample. The shrinkage of the sample sintered under zero stress is seen to be almost isotropic.

Within the limits of experimental error, the applied stress caused no change in the density; the final density of the sample sintered under the initial stress of $0.2 \mathrm{MPa}$ was 0.97 compared with 0.96 for the sample sintered under zero stress. The deformation of the sample under the action of the applied stress was therefore constant volume creep.

Figure 3.4 shows a plot of $\epsilon_{z}$ versus $\epsilon_{r}$ for samples sintered between $500{ }^{\circ} \mathrm{C}$ and $1000^{\circ} \mathrm{C}$ under constant heating rate conditions of $2^{\circ} \mathrm{C} / \mathrm{min}, 4^{\circ} \mathrm{C} / \mathrm{min}$, and $8^{\circ} \mathrm{C} / \mathrm{min}$ at a constant load of 7 Newton. Figures $3.5 \mathrm{a}, \mathrm{b}$, and $\mathrm{c}$ show the microstructures at a constant relative density of approximately 0.70 .

- true strain - In(length / original length), from now on true strain will be referred to as the strain. 


\subsubsection{Aluminum Oxide}

Figure 3.6 shows the axial true strain, $\varsigma_{z}$, versus the radial true strain, $\epsilon_{r}$, for the $\mathrm{Al}_{2} \mathrm{O}_{3}$ powder compacts. Line $\mathrm{A}$ corresponds to the constant heating rate experiments, while line $B$ corresponds to the isothermal experiments, both at an applied load of $7 \mathrm{~N}$. Line B does not go through the origin, since some shrinkage has already occurred in the heat-up period, before isothermal conditions were reached and the load was applied. Line $\mathrm{C}$ corresponds to the zero load sintering.

\subsection{Analysis}

In this section the densification strain rate, $\dot{\epsilon}_{d}$, and the creep strain rate, $\dot{\epsilon}_{c}$, are related to the axial-strain/radial-strain plot, $\epsilon_{z}$ versus $\epsilon_{r}$. From the analysis, some simplified experimental procedures emerge.

For a body loaded uniaxially and deforming uniformly it is readily shown that the linear densification strain is

$$
3 \epsilon_{d}=\epsilon_{z}+2 \epsilon_{r}
$$

Eqn. 3.2

while at the same time, for the total axial strain, $\epsilon_{z}$ :

$$
\epsilon_{z}=\epsilon_{c}+\epsilon_{d}
$$

Eqn. 3.3

from which

$$
\epsilon_{c}=\frac{2}{3}\left(\epsilon_{z}-\epsilon_{r}\right)
$$


where the meaning of the various strains is indicated in Fig. 3.7.

If the creep strain at constant uniaxial stress is linearly proportional to the densification strain then one must expect that

$$
\epsilon_{z}=k \epsilon_{r}
$$

Eqn. 3.5

The relative densification and creep strains as shown in Fig. 3.6 can be obtained from dilatomcter data in Figs. 3.2 and 3.3, and density measurements.

The densification strain rate, $\dot{\epsilon}_{d}$, and the creep strain rate, $\epsilon_{c}$, can be obtained as a function of temperature by fitting smooth curves through the data of Fig. 3.8 and differentiating:

$$
\begin{array}{ccc}
\dot{\epsilon}_{d}=\frac{\dot{\rho}}{3 \rho}=\frac{4}{3 \rho} \frac{d \rho}{d T} & \text { Eqn. 3.6 } \\
\dot{\epsilon}_{c}=4 \frac{d \dot{\epsilon}_{c}}{d T} & \text { Eqn. 3.7 }
\end{array}
$$

$\rho$ is the instantaneous density, $T$ is the temperature, and the factor 4 arises from the constant heating rate of $4^{\circ} \mathrm{C}$ per minute that was used in the experiments when time is expressed in minutes.

Eqns. 3.4 and 3.5 are also valid as strain rate equations so that

$$
\frac{\dot{\epsilon}_{d}}{\dot{\epsilon}_{c}}=\frac{(k+2)}{2(k-1)}
$$


At fixed density, i.e. $\dot{p}=0$, constant volume creep requires that

$$
\epsilon_{c}=-2 \epsilon_{r c}
$$

where $\epsilon_{r c}$ is the radial component of the creep strain. The locus of $\epsilon_{z}$ at some fixed density in a plot of $\epsilon_{z}$ versus $\epsilon_{r}$ is then a straight line with a slope of $1 / 2$, as shown in Fig. 3.9a. The data for constant load creep should then follow a straight line in the $\epsilon_{z} / \epsilon_{r}$ plot and intersect a constant density line when the sintered density with applied uniaxial stress equals that of the sintered density at zero load. This intersection for $\mathrm{ZnO}$ (see Fig. 3.9a) shows at once that creep indeed occurs at nearly constant volume. Similar results are obtained for $\mathrm{Al}_{2} \mathrm{O}_{3}$. Near constant volume creep has been observed for all ceramics studied so far by low-load loading dilatometry. It is then possible, with considerable confidence, to identify the $\epsilon_{r}$ value corresponding to a measured shrinkage strain, $\epsilon_{z}$, for a particular density, by making a construction as shown in Fig. 3.9b, without the need of measuring $\epsilon_{r}$ directly.

As seen from Eqn. 3.8, the ratio of $\dot{\epsilon}_{d} / \dot{\epsilon}_{c}$ is very sensitive to variations in the values of $k$. In the actual experiment, the data are collected at constant load rather than at constant stress. Since the sample cross sectional area, A, changes from the initial one, $A_{0}$, as a result of densification and creep, the applied uniaxial stress, $\sigma$, differs from the initial stress, $\sigma_{0}$ :

$$
\sigma=\frac{\sigma_{0} A_{0}}{A}
$$

An estimate can be made readily of how much change in $k$ would be associated with the changing stress for the experimental conditions adopted here, or what the 
error would be if the constant load experiment is simply treated as if it were at constant stress. To allow for this estimate the total strains are needed, and for the sake of illustration a simple creep relationship, which materials such as $\mathrm{ZnO}$ obey well for a large part of the densification, will be adopted:

$$
-\epsilon_{c}=B o \ln (t)
$$

Eqn. 3.11

and differentiating,

$$
-\dot{\epsilon}_{c}=\frac{B \sigma}{t}=\frac{B_{c} \sigma_{o}}{t} \frac{A_{o}}{A}
$$

where $\mathrm{B}$ is a kinetic constant, $\sigma$ is the applied uniaxial stress, $\mathrm{A}$ is the cross-sectional area, and $t$ is the time. Also one has:

$$
\frac{A_{0}}{A}=\left(\frac{R_{0}}{R}\right)^{2}=\exp \left(-2 \epsilon_{r}\right)
$$

where $R_{O}$ and $R$ are the initial and the instantaneous radius of the sample cross section. To a first approximation the creep strain rate may be expressed as

$$
-\dot{\epsilon}_{c}=\frac{\sigma_{o} B\left(1-2 \epsilon_{r}\right)}{t}
$$

At low loads, the creep strain has been found to be much smaller than the densification strain (typically $\epsilon_{c}<0.2 \epsilon_{d}$ ). Then, with $\epsilon_{r} \approx \epsilon_{d}$, integration yields 


$$
\epsilon_{c}^{\prime}(t, \sigma)=\epsilon_{c}\left(t, \sigma_{o}\right)\left(1+\epsilon_{d}\right)
$$

Eqn. 3.15 can be used to correct $k$ to constant stress. The necessary information is shown in Fig. 3.10 where line 1 is the isotropic line, line 2 is the constant load line, and line 3 is the constant density line. The correction to each $\epsilon_{r}$ and $\epsilon_{z}$ datum point for $\mathrm{ZnO}$ is shown in Fig. 3.11.

The values of $\mathrm{k}$ that follow from such an analysis are 1.33 for $\mathrm{ZnO}$, and 1.29 and 1.30 for $\mathrm{Al}_{2} \mathrm{O}_{3}$ done under constant heating rate and isothermal conditions, respectively. Using Eqn. 3.8 this then yields values of the densification strain rate over the creep strain rate as 5.00 for $\mathrm{ZnO}$, and 5.67 and 5.50 for $\mathrm{Al}_{2} \mathrm{O}_{3}$, all at a stress of 0.2 MPa. Data can also be obtained from the creep strain rate (converted to constant stress rates by compensating for the cross-sectional area change) and densification strain rate measurements, rather than from the total strain determination. The ratios of $\dot{\epsilon}_{d} / \dot{\epsilon}_{c}$ obtained from the rate measurements are 4.5 - 5.0 for $\mathrm{ZnO}$ and 5.7-6.5 for $\mathrm{Al}_{2} \mathrm{O}_{3}$. The data for $\mathrm{ZnO}$ are shown in Fig. 3.12. The two methods for obtaining $\dot{\epsilon}_{d} / \dot{\epsilon}_{c}$ are in close agreement. This shows that the total strain determination is useful for generating values of $\dot{\epsilon}_{d} / \dot{\epsilon}_{c}$ with minimum experimentation.

As seen in Fig. 3.4 all the $\left(\epsilon_{z}, \epsilon_{r}\right)$ values for $\mathrm{ZnO}$ with an initial relative density of 0.53 sintered with ccnstant heating rate conditions of $2^{\circ} \mathrm{C}, 4^{\circ} \mathrm{C}$, and $8^{\circ} \mathrm{C} / \mathrm{min}$ all fell on a single line. The value of $k$ is 1.15 , which gives a value for $\dot{\epsilon}_{d} / \dot{\epsilon}_{c}$ of approximately 10.5 . 


\subsection{Discussion}

The discussion is divided into three sections. First, the general implication of the densification and creep strain rate ratio to the sintering stress is considered. Then, strain rates equations are derived rigorously under ideal conditions. Finally, the densification ard creep viscosity ratio for real systems and its implication to sintering are discussed.

\subsubsection{Densification Strain Rate}

In general rate expressions are written as a product of two components, a kinetic term and a driving force term. Similarly, the densification strain rate equation can be written as a viscosity term, which includes the various kinetic terms and the microstructural terms, times a driving force term, which include both the internal driving force, $\Sigma$, and the externally applied driving force, $\sigma_{a}$.

$$
\dot{\epsilon}_{d}=\frac{1}{\eta_{d}}\left(\Sigma+\sigma_{a}\right)
$$

where $\dot{\epsilon}_{d}$ is the densification strain rate, $\eta_{d}$ is the densification viscosity, $\Sigma$ is the sintering stress, and $\sigma_{a}$ is the external applied hydrostatic pressure which is equal to one third of the uniaxially applied stress, $\sigma$.

By analogy the creep strain rate for a deforming material is written

$$
\dot{\epsilon}_{c}=\frac{1}{\eta_{c}} \sigma
$$


where $\dot{\epsilon}_{c}$ is the creep strain rate, $\eta_{c}$ is the creep viscosity, and $\sigma$ is the applied uniaxial pressure.

The densification strain rate over the creep strain rate gives

$$
\frac{\dot{\epsilon}_{d}}{\dot{\epsilon}_{c}}=\frac{\eta_{c}}{\eta_{d}}\left(\frac{\Sigma}{\sigma}+\frac{1}{3}\right)
$$

By measuring $\dot{\epsilon}_{d} / \dot{\epsilon}_{c}$, the behavior of the viscosity ratio and the sintering stress can be inferred. Figure 3.12 shows the ratio of the densification strain rate over the creep strain rate for $\mathrm{ZnO}$. This ratio is nearly constant as a function of temperature, for the ceramics examined here: $\mathrm{ZnO}$ and $\mathrm{Al}_{2} \mathrm{O}_{3}$. The results of the isothermal loading dilatometry in Fig. 3.1 demonstrate that the creep strain rate over the constant-stress densification strain rate is also insensitive to density changes for a particular system. In addition, as seen in the $\mathrm{Al}_{2} \mathrm{O}_{3}$ experiments, the densification strain rate over the creep strain rate is equal in this experimental case for both the isothermal condition and the constant heating rate condition. Therefore, the densification over the creep strain rate can be obtained reliably from a non-isothermal experiment, extending the measurement range to the earliest stages of densification. Deviations of the proportionality between the constant stress creep strain rate and the simultaneous densification strain rate would be manifested by the $\epsilon_{z}$ vs. $\epsilon_{r}$ data not extrapolating through the origin. Such a deviation is not apparent in Figs. 3.6 or 3.11; thus $\dot{\epsilon}_{d} / \dot{\epsilon}_{c}$ is nearly constant from the onset of sintering. The final stage of densification remains unexplored to date, since the density change is very slow and therefore accurate measurements become difficult to obtain. 
Creep and sintering experiments (Fig. 3.1) now have shown that $\dot{\epsilon}_{d} / \dot{\epsilon}_{c}$ remains relatively constant from the onset of densification to the final stages of sintering for the systems executed so far. For a specific system this ratio usually does not vary by more than a factor of 1.5 .

The only significant change in the strain rate ratio for compacts made from the same powder results from a change in the initial density. While the value of the ratio still remains fairly constant for the specific system; the compact with the higher initial density has a higher densification/ creep strain rate ratio than the compact with the lower initial density. This trend can be expected since, when compared at a constant density, compacts with a higher starting density will have a smaller grain size. Since the sintering stress is expected to scale with the inverse of the structure's scale (see Eqn. 2.4) the compact with a smaller grain size will have a higher driving force.

The constancy of the ratio of $\dot{\epsilon}_{d} / \dot{\epsilon}_{c}$ can result either from $\eta_{c} / \eta_{d}$ and $\Sigma$ both being constant or by the two factors compensating for each other. Since a compensation mechanism is unlikely and measurements of the viscosity ratio show little, if any, change [Rahaman et al 1988, De Jonghe and Srikanth 1988] the constancy in the strain rate ratio is a strong indication that the sintering stress, $\Sigma$, is constant within the same density range. This change is considerably less than what v/ould be expected on the basis of most models (see section 2.2.1) which predict an increase in the sintering stress as the material densifies.

\subsubsection{Model Derivation of Strain Rate Equation}

An equation for the densification strain rate of a system can be derived rigorously under ideal conditions of uniform particle size and packing distribution. Although real 
powder systems are far from ideal and always have variations in size and packing, the derived densification strain rate equation gives a guide to the minimum number of parameters necessary to describe the densification behavior.

\subsubsection{Grain Boundary Diffusion}

The derivation of the linear densification strain rate, $\dot{\epsilon}_{d}$, for spherical particles, with grain boundary diffusion as the mechanism is shown in the following paragraphs.

The geometry of the particles and the grain boundary is shown in Fig. 3.13. The flux as a function of a radius, $r$, is

$$
F(r)=\frac{-D_{b} \nabla \mu}{\Omega k T}
$$

where $D_{b}$ is the grain boundary diffusion coefficient, $\Omega$ is the atomic volume, $k$ is Boltzman's constant, $\mathrm{T}$ is the absolute temperature, and $\mu$ is the chemical potential.

The total number of atoms crossing along the circumference at the radius per unit time is given by

$$
J(r)=-\frac{2 \pi r \delta D_{b} \nabla \mu}{\Omega k T}
$$

where $\delta$ is the grain boundary thickness.

The rate of approach of the two sphere centers, du/dt, is related to $J(r)$ by:

$$
J(r)=\frac{\pi r^{2}}{\Omega} \frac{d u}{d t}
$$


since the displacement rate at the boundary must be independent of $r$.

From Eqns. 3.20 and 3.21,

$$
\frac{d \mu}{d r}=-2 A r
$$

$\mathrm{A}$ is a constant. From integrating Eqn. 3.22 one obtains

$$
\mu=-A r^{2}+B
$$

Eqn. 3.23

Since $\mu=\Omega \sigma$, where $\sigma$ is the stress on the boundary, Eqn. 3.23 can be rewritten as

$$
\sigma=\frac{B-A r^{2}}{\Omega}
$$

From the boundary conditions, A and B can be found. The first boundary. condition is that the stress at the edge of the neck is equal to zero, i.e. $\sigma(R)=0$, and the second condition is that the average stress on the grain boundary,

$$
\sigma_{\text {ave }}=\int_{0}^{2 \pi} \int_{0}^{R} \frac{(\sigma(r)) r d r d \theta}{\pi R^{2}}=\sum \phi \text { Eqn. 3.25 }
$$

where $\Sigma$ is the sintering stress and $\phi^{*}$ is the stress intensification factor. If all the internal driving force of the system were removed then $\Sigma$ can be thought of as the

* $\phi$ - the total area / grain boundary area (see Fig. 2.5 and section 4.4.1) 
externally applied stress necessary to attain the same densification strain rate as when the system had its actual driving force. By solving for $\mathrm{A}$ and $\mathrm{B}$, the total flux coming out at the edge of the neck is

$$
J(R)=\frac{8 \pi D_{b} \delta \sum \phi}{k T}
$$

To relate the flux at the neck between two particles to the shrinkage of the body, consider spherical particles in a simple cubic cell arrangement (Fig. 3.14). The total vrlume transported out of one neck in the time interval $\Delta t$ is equal to

$$
\frac{J(R) \Omega \Delta t}{2}=\frac{\Delta m \pi R^{2}}{2}
$$

$\Delta m$ is the change in the corresponding cell center-to-center distance, $R$ is the neck radius, and the factor of 2 arises because each neck is shared by 2 cells. Volumetric shrinkage occurs in all three directions so that

$$
\frac{\Delta V}{V}=\frac{\Delta l}{l}+\frac{\Delta m}{m}+\frac{\Delta n}{n}
$$

Since $V=l m n$, the rate of change in the cell volume is

$$
\frac{d V}{d t}=\frac{\Delta V}{\Delta t}=\Omega J\left(\frac{m n}{\pi R^{2}}+\frac{l n}{\pi R^{2}}+\frac{l m}{\pi R^{2}}\right)_{\text {Eqn. } 3.29}
$$

By definition, the cell external surface area divided by the neck area is equal to $\phi$, so that Eqn. 3.29 is equal to 


$$
\frac{d V}{d t}=3 \phi \Omega J
$$

since one atom of volume $\Omega$ removed from a boundary reduces the cell volume by $\phi \Omega$.

The instantaneous volumetric strain rate of the cell is obtained by dividing $d V / d t$ by the cell volume, $\mathrm{V}$, which is equal to $l m n$. Taking $l=m=n=\mathrm{x}$, where $\mathrm{x}$ is also the center-to-center distance between the pores,

$$
\frac{1}{V} \frac{d V}{d t}=\frac{3 \phi \Omega J}{x^{3}}
$$

The linear densification strain rate, $\dot{\epsilon}_{d}$ is equal to $-(1 / 3 \mathrm{~V})(\mathrm{dV} / \mathrm{dt})$ so that

$$
\dot{\epsilon}_{d}=\frac{8 \pi D_{b} \delta \Omega \phi^{2} \Sigma}{x^{3} k T}
$$

\subsubsection{Volume Diffusion}

The linear densification strain rate for volume diffusion can be obtained from Eqn. 3.32 by substituting $2 R$ for $\delta$ and $D_{v}$ for $D_{b}$ [Raj and Ashby 1975]. Since $\phi$ is equal to $x^{2} / R^{2}$

$$
\dot{\epsilon}_{d}=\frac{16 \pi D_{v} \Omega \phi^{\frac{3}{2}} \Sigma}{x^{2} k T}
$$

\subsubsection{General Isothermal Densification Strain Rate Equation}

Equation 3.32 and 3.33 may be written in the general form: 


$$
\dot{\epsilon}_{d}=\frac{H D \phi^{\frac{n+1}{2}}\left(\Sigma+\sigma_{a}\right)}{x^{n} k T}
$$

where $\mathrm{H}$ is a constant, $\mathrm{D}$ is the diffusion coefficient, $\Sigma$ is the sintering stress and $\sigma_{a}$ is an additional hydrostatic applied stress as, for example, from hot pressing ( $\sigma_{a}=0$ for free sintering), $\phi$ is the stress intensification factor, $\mathrm{n}=3$ for grain boundary diffusion and $\mathrm{n}=2$ for volume diffusion, $\mathrm{x}$ is the center-to-center pore spacing, $\mathrm{k}$ is Boltzman's constant, and $\mathrm{T}$ is the absolute temperature.

\subsubsection{Creep Strain Rate}

When the applied stress induces creep without biasing densification, the mechanisms for creep and densification are the same [Rahaman and De Jonghe 1984]. Therefore, parallel with Eqn. 3.34 the creep strain rate is written as

$$
\dot{\epsilon}_{c}=\frac{H^{\prime} D \phi^{\frac{n+1}{2}} \sigma}{x^{n} k T}
$$

where $\sigma$ is the uniaxially applied stress $\left(\sigma_{a}=\frac{1}{3} \sigma\right)$ and $\mathrm{H}^{\prime}$ is a constant.

\subsubsection{Ratio of Densification Strain Rate / Creep Strain Rate}

As evident from Eqns. 3.34 and 3.35, the densification strain rate over the creep strain rate is

$$
\frac{\dot{\epsilon}_{d}}{\dot{\epsilon}_{c}}=K\left(\frac{\Sigma}{\sigma}+\frac{1}{3}\right)
$$


where $\mathrm{K}$ is a constant and $\sigma$ is the uniaxiall $\mathrm{V}$ pplied stress. When Eqn. 3.36 is compared to Eqn. 3.18, it is seen that under ideal conditions the viscosity ratio is a constant, therefore the strain rate ratio is a directly proportional to the sintering stress.

As evident in Fig. 3.1 the sintering stress under isothermal conditions for a given system is fairly constant as a function of the sintered density. In addition, from the non-isothermal experiments, as a function of temperature, the sintering stress does not change as a function of temperature. Specifically, the results obtained with $\mathrm{Al}_{2} \mathrm{O}_{3}$ show that the sintering stress for both isothermal and constant heating rate are the same, extending the range of the determination of the sintering stress to lower densities.

\subsubsection{Densification Viscosity / Creep Viscosity}

The general strain rate equations derived in the previous section indicate that the viscosity ratio of a system should remain constant. It is instructive to see how this ratio behaves in real systems.

The ratio of the creep viscosity over the densification viscosity does not follow directly from the measured values of the densification strain rate over the creep strain rate. To obtain these values from loading dilatometry experiments, it will be necessary to measure precisely the small density increases that result from the hydrostatic component of the applied uniaxial stress. In principle, this should be possible, since the hydrostatic component of the applied stress, $\sigma_{a}$, can be added simply to the sintering stress , $\Sigma$ :

$$
\dot{\epsilon}_{d}(\sigma)=\frac{\sum+\frac{\sigma}{3}}{\eta_{d}}=\dot{\epsilon}_{d}(\sigma=0)+\frac{\sigma}{3 \eta_{d}} \quad \text { Eqn. } 3.37
$$


so that with

$$
\dot{\epsilon}_{c}=\frac{\sigma}{\eta_{c}}
$$

one obtains

$$
\frac{\eta_{c}}{\eta_{d}}=3\left(\frac{\dot{\epsilon}_{d}(\sigma)-\dot{\epsilon}_{d}(\sigma=0)}{\dot{\epsilon}_{c}}\right)
$$

The measurements presented here were not sufficiently accurate to determine whether this ratio is indeed constant throughout the densification process. In several liquid phase sintering systems, accurate measurements showed that the ratios indeed remained fairly constant throughout the intermediate stage of densification, and were less than 2 [De Jonghe and Srikanth 1988, Rahaman et al 1988]. Theoretical consideration by Bordia and Scherer [1988] lead to the conclusion that the ratio of the creep viscosity over the densification viscosity should be less than 1.5 . Further, the constancy of the densification strain rate over the creep strain rate would require some unlikely compensation mechanisms to be at work if the viscosity ratio were not constant. All these consideration lead to the conclusion that both the sintering stress, $\Sigma$, and the creep viscosity over the densification viscosity, $\eta_{c} / \eta_{d}$, are constant from the very early stages to late into the intermediate sintering stage.

The sintering stress could be constant if the pore size decrease due to densification is approximately compensated by the pore size increase due to coarsening. In systems in which coarsening is suppressed, e. g. monosized particles, the sintering stress would have to increase with density. The results of Gregg and Rhines [1973] on large, monosized copper spheres would be in this category and their measurement show an 
increase in the sintering force. In addition the driving force for sintering should include both the curvatures of the pore and grain size [Cannon 1981, Raj 1987], so even though the decreasing size of the pores should increase densification the increasing grain size would slow the process. Finally, in a conventional powder compact, a multitude of densification states are present simultaneously which makes the pore structure very complicated [Barrett and Yust 1967]. The shift from open porosity to closed porosity, for example, does not take place suddenly, but develops gradually throughout the densifying compact [Coleman and Beere 1975]. This would also tend to average changes in the sintering stress and thereby smooth the changes [De Jonghe et al 1989].

\subsection{Summary}

The results of the present study show that, when constant volume creep deformation occurs during sintering, the ratio of the densification to the creep strain rate is almost independent of temperature and heating rate.

A model based on a homogeneous system shows that the densification and creep strain rate ratio is a direct measure of the sintering stress.

Within the useful temperature range for densification, the ratio of the densification to the creep viscosity is nearly independent of temperature.

The present data have important implications for the sintering of heterogeneous ceramics and composites. The matrix densification strain rate of a particulate composite containing a fixed volume fraction of inclusions is predicted to increase with increases of the densification to the creep viscosity ratio. However, since the viscosity ratio appears to be independent of temperature and heating rate; an effective method to improve sintering of composites is to improve sintering of the matrix phase. 


\title{
4 EFFECT OF HEATING RATE ON SINTERING AND COARSENING
}

\begin{abstract}
The sintering of zinc oxide powder compacts has been investigated at constant rates of heating of $0.5-15^{\circ} \mathrm{C} / \mathrm{min}$. For samples with the same initial relative density of 0.50 , the temperature derivative of the densification strain versus density fits within a single, relatively narrow band. At low temperatures the densification strain rate as a function of temperature increases almost linearly with the heating rate. The data. covering a wide density range of $0.5-0.98$, are consistent with an analysis that accounts for the coarsening, (defined as an increase in the mean pore separation) in terms of two classes of microstructural coarsening processes: those associated with densifying and with non-densifying mechanisms.
\end{abstract}

\subsection{Introduction}

An important processing goal for technical ceramics is obtaining a uniform microstructure, at high density with desired grain size. To obtain this goal a range of experimental techniques, such as hot pressing or heating with varied time-temperature schedules for the sintering process, are available. In this chapter the effects of particular time-temperature schedules, constant heating rates, are examined. Such non-isothermal processing steps are quite important since they occur, to varying degrees of approximation, in all sintering processes. Even a so-called isothermal sintering has a heat-up period in which considerable microstructural development and densification can occur; frequently, more than half of the possible densification of a ceramic compact is reached at the end of this heat-up phase, regardless of how rapidly it is executed. In several instances, very rapid heating, also called fast firing, has produced beneficial effects in maintaining relatively fine microstructures at high sintered densities [Brook 1982, Johnson 1984]. The heating rate is thus an intrinsic 
part of sintering, either where used as a parameter to influence the course of microstructur evolution or as a contribution to the starting conditions for conventional, constant-temperature processing.

Much work on the effect of heating rate on sintering was conducted fifteen to twenty years ago. Typically, data for the initial stage sintering of amorphous or polycrystalline powder compacts were correlated with data obtained under isothermal sintering conditions. Only limited success in the correlation of data and model was achieved for polycrystalline systems. This was primarily due to the difficulties in analyzing sintering data when several transport mechanisms were active simultaneously. Cutler [1969] studied the initial sintering of spherical glass powder compacts at constant rates of heating from $0.4-2.9^{\circ} \mathrm{C} / \mathrm{min}$ and compared the data with the predictions of Frenkel's model [1945]. Good agreement between theory and experiment was obtained if the viscosity of the glass was described by a thermally activated process with a fixed activation energy. The initial stage sintering of polycrystalline powder compacts has been studied by Young and Cutler [1970], who analyzed their data in terms of an equation developed by Johnson [1969] for the sintering of two spherical particles by simultaneous volume, grain-boundary, and surface diffusion. Young and Cutler suggested that, in constant heating rate experiments, there may be distinct temperature regions in which grain-boundary or volume diffusion may dominate over surface diffusion. This behavior was, however, not readily apparent from the data. Woolfrey and Bannister [1972] formulated techniques for analyzing the initial stage shrinkage during constant heating rate sintering and concluded that, in principle, their techniques permit the determination of all the sintering parameters normally obtained from isothermal sintering data. Data for the rate law, diffusion coefficient, and activation energy obtained from constant heating rate and isothermal sintering of $\mathrm{UO}_{2}$ and $\mathrm{ThO}_{2}$ showed good agreement. 
Woolfrey and Bannister's results might be considered to be fortuitous since, as in the work of Young and Cutler a central issue, i.e. the simultaneous occurrence of more than one sintering mechanism was not addressed.

Recently, Lange [1989] studied the sintering of $\mathrm{Al}_{2} \mathrm{O}_{3}$ powder compacts with the same initial density at constant rates of heating of $2.5-20^{\circ} \mathrm{C} / \mathrm{min}$ up to $1500^{\circ} \mathrm{C}$. For each heating rate, the shrinkage strain rate increased to a maximum and then decreased. The maximum densification strain rate occurred at approximately the same relative density of 0.77 for each heating rate. Lange suggested that sintering kinetics dominated the densification process up to a relative density of 0.77 after which coarsening kinetics dominated. In contrast to the earlier work discussed above that studied the initial stage of sintering only, the data of Lange covered almost the entire densification process. It is interesting to note here that the maximum densification strain rate occurred at densities where, under isothermal conditions, logarithmic densification is observed.

It is generally accepted that the critical issue in microstructure development is the interplay between densification and coarsening [Brook 1982]. Usually, coarsening is described as the evolution of grain size. While for dense materials this is undoubtedly the feature of importance, model-based descriptions of densification strain rates should be based on the mean interpore separation. A convenient assumption is to put the grain size about equal to the pore separation (see section 2.3), but a microstructural examination of most partly-sintered compacts reveals that this is not a very accurate assumption. With respect to the densification strain rates, it is proposed that the relevant coarsening parameter is the increase in the mean pore-separation, $x$ (see section 3.5.2). With this definition, and with a distribution of pore sizes in a given matrix, two classes of processes can be recognized as contributing to coarsening: non-densifying processes, such as ones governed by surface diffusion and evaporation/ 
condensation where smaller pores disappear by depositing themselves on larger pores, and densifying processes, such as the ones brought about by grain boundary and volume diffusion where the smaller pores disappear as a consequence of overall densification. If the temperature dependence of these two classes of coarsening mechanisms are considered, then at least two different activation energies are necessary to describe the temperature dependence of coarsening. One objective of this chapter is to show the influence of the heating rate under conditions where the two coarsening processes are effective.

Earlier work by De Jonghe and co-workers [Raharnan and De Jonghe 1987b, Chu et al 1989] indicated that $\mathrm{ZnO}$ and $\mathrm{CdO}$ can be useful as model oxides for isothermal sintering studies. It was found for these, as well as for a number of other ceramirs and glasses, that the ratio of the densification strain rate to the constant-stress creep rate remains constant, from the onset of densification to relative sintered densities in excess of 0.9 (Fig. 3.1). For $\mathrm{ZnO}$ [Chu et al 1989] this constancy of the ratio was also observed under conditions of a constant heating rate of $4^{\circ} \mathrm{C} / \mathrm{min}$. Subsequent experiments following the same procedures with heating rates of $2^{\circ} \mathrm{C} / \mathrm{min}$ and $8^{\circ} \mathrm{C} / \mathrm{min}$ also showed a constant ratio of the densification strain rate to the constant stress creep strain rate. This constancy leads to the conclusion that the sintering stress, which is related to this ratio, is likely to be constant under similar conditions. The present chapter examines the non-isothermal densification behavior of $\mathrm{ZnO}$ powder compacts at constant heating rates between 0.5 and $15^{\circ} \mathrm{C} / \mathrm{min}$. All compacts had the same initial relative density of $0.50 \pm 0.01$, and the experiments were terminated at $1100^{\circ} \mathrm{C}$, when the samples reached relative densities in excess of 0.96 . The analysis, using relatively simple and model unspecific rate expressions, predicts qualitatively the major effects of the densification behavior, such as the insensitivity of both the densification strain and the 
temperature derivative of the densification strain to the heating rate. In addition, the analysis shows the need to include separate coarsening processes with different activation energies to account for the data obtained at various heating rates.

\subsection{Experimental Procedure*}

$\mathrm{ZnO}$ powder compacts $(6 \mathrm{~mm}$ diameter by $6 \mathrm{~mm}$ ) of the same initial relative density of $0.50 \pm 0.01$ were made by uniaxial compression of the powder at $\sim 20 \mathrm{MPa}$ in a tungsten carbide die. Constant heating rate sintering was performed in a dilatometer at five imposed heating rates ranging from 0.5 to $15^{\circ} \mathrm{C} / \mathrm{min}$. The same procedure was used in all sintering runs; the sample was heated at $10^{\circ} \mathrm{C} / \mathrm{min}$ from room temperature to $500^{\circ} \mathrm{C}$ (below which temperature no densification could be detected) followed by a controlled heating rate to $1100^{\circ} \mathrm{C}$, after which the sample was cooled rapidly. The shrinkage of a standard quartz sample was measured under identical conditions in order to correct for differences in shrinkage between the dilatometer ${ }^{* *}$ rod and the sample holder. The density of the $\mathrm{ZnO}$ sample was determined from its initial density and the measured shrinkage. The microstructure of selected samples was observed by scanning electron microscopy of polished and etched surfaces. Additionally, the final average grain size of the samples was measured.

\subsection{Results}

Figure 4.1 shows results for the relative density, $\rho$, vs. temperature, $T$, for samples with the same initial relative density of $0.50 \pm 0.01$ and sintered at constant rates of heating ranging from 0.5 to $15^{\circ} \mathrm{C} / \mathrm{min}$. The data for any heating rate are reproducible

- Most of the experiments described in this chapter were performed by M.N. Rahaman.

* Harrop Industries, Inc., Columbus, $\mathrm{OH}$ 
to within $\pm 1 \%$. The curves have the familiar sigmoidal shape and, generally, are shifted to higher temperatures with increasing heating rate; the final densities are high $(0.96$ $-0.98)$. It can be noted that the achieved sintered densities at any temperature showed a modest but systematic dependence on the heating rates.

The temperatures derivative of the densification strain, $(1 / \rho) d \rho / d T$, is plotted in Fig. 4.2 as a function of $\rho$ for the different heating rates. The data fall into a common band for the relatively wide range of heating rates used. (Much higher heating rates could not be used with the existing equipment; in addition, higher heating rates can be expected to lead to problems with thermal gradients in the sample. Much lower heating rates are very time consuming and difficult to control accurately.)

Figure 4.3 shows the data for the volumetric strain rate, $(1 / \rho) \mathrm{d} \rho / \mathrm{dt}$, plotted as a function of temperature for the heating rates used. (The linear densification strain rate is equal to one third of the volumetric strain rate.) The maximum of the curves shifts slightly to higher temperature with increasing heating rate. Above $\sim 700^{\circ} \mathrm{C}$ the densification strain rate at a fixed temperature increases approximately linearly with heating rate; however, below this temperature the increase in densification strain rate is lower. It can also be noted that in the early stages of sintering $\left(\sim 550^{\circ} \mathrm{C}\right)$ the densification strain rate is not independent of heating rate; moreover, if the curves are extrapolated to $500^{\circ} \mathrm{C}$ (at which temperature measurable shrinkage begins) they do not converge to a single densification strain rate.

The final grain sizes of the samples sintered at constant heating rates of $0.5^{\circ} \mathrm{C} / \mathrm{min}, 2^{\circ} \mathrm{C} / \mathrm{min}, 5^{\circ} \mathrm{C} / \mathrm{min}$ and $15^{\circ} \mathrm{C} / \mathrm{min}$ are $9.6 \pm 1.8 \mu \mathrm{m}, 7.2 \pm 0.9 \mu \mathrm{m}, 5.9 \pm$ $0.5 \mu \mathrm{m}$, and $5.0 \pm 0.7 \mu \mathrm{m}$ respectively. Scanning electron micrographs of the samples 
for $5^{\circ} \mathrm{C} / \mathrm{min}$ and $0.5^{\circ} \mathrm{C} / \mathrm{min}$ are shown in Figs. $4.4 \mathrm{a}$ and $\mathrm{b}$, respectively; both samples were heated to $1100^{\circ} \mathrm{C}$ and then cooled. The starting particle size of the $\mathrm{ZnO}$ starting powder was $\sim 0.1 \mu \mathrm{m}$.

In Fig. 4.5 the cube of the average grain sizes of samples sintered at various heating rates is plotted versus the inverse of the heating rate, $\alpha^{-1}$. The significance of this figure will be discussed in section 4.4.1.

\subsection{Discussion}

The discussion has been divided into three sections. First, an equation for the sintering and microstructural evolution is developed. Next, special microstructural conditions of no coarsening and extreme coarsening are considered. Finally, numerical calculations and experimentally based values of the pore spacing are compared.

\subsubsection{Model}

The results of sintering at constant heating rates should follow from an appropriate sintering and microstructural evolution model and its constitutive equations. In this section the results will be interpreted in the context of an expression for the densification strain rates as well as for the microstructural evolution. The expressions should conform to the multitude of empirical observations.

The instantaneous isothermal, linear densification strain rate for an ideal monosize system, $\dot{\epsilon}_{d}$, as derived in chapter 3 (Eqn. 3.34), is

$$
\dot{\epsilon}_{d}=\frac{1}{3 \rho} \frac{d \rho}{d t}=\frac{H D \sum \phi^{\frac{n+1}{2}}}{x^{n} k T}
$$


here $\rho$ is the instantaneous density, $\mathrm{H}$ is a constant, $\mathrm{D}$ is the volume diffusion coefficient, $\sum$ the sintering stress, $\phi$ is the stress intensification factor, $\mathrm{x}$ is an average interpore distance, $\mathrm{k}$ is the Boltzmann factor, $\mathrm{T}$ is the absolute temperature, and $\mathrm{n}$ depends on the mass transport mechanism. The mean grain boundary stress for free sintering is then $\phi \Sigma$. The term $\phi$ is of geometrical origin [Beere 1978a\&b] and is akin to the efficiency factor introduced by DeHoff [1984]. Real powder systems always have associated size and packing distributions which are far from ideal, but the derived densification strain rate equation gives a guide to the minimum number of parameters necessary to describe the densification behavior.

At a given temperature most terms in Eqn. 4.1 are constant, the time-varying terms are the stress intensification factor, $\phi$, the interpore spacing, $x$, and the sintering stress, $\Sigma$.

Theoretically, the sintering stress, $\Sigma$, should be proportional to the average of $1 / r$, where $r$ is the pore radius, and could be expected to change with the density of the material. In sintering studies with large monosized copper spheres, where densification occurs without much grain growth, $\Sigma$ does indeed increase with increasing density [Gregg and Rhines 1973]. This ideal behavior is evident in restrictive cases where the particle sizes and packing can be controlled. In contrast, numerous constant-stress creep and densification experiments with commercially available powders observe $\Sigma$ to remain constant as sintering proceeds, from the onset of densification to well into the later stages [Chu et al 1989]. These commercial powders have a wide range of sizes and packing heterogeneity. Although constant, the value of $\Sigma$ has been found to depend only on the initial powder compact state. In these 
systems the constancy of $\Sigma$ will be taken as sufficiently documented; and therefore the only terms that change as a result of sintering in the densification strain-rate equation (Eqn. 4.1) are $\phi$, the stress intensification factor, and $x$, the interpore spacing.

The stress intensification factor, $\phi$, is defined as the total area of the grain boundary and the pore divided by the area of the grain boundary. $\phi$ approaches 1 as the sample approached full density. $\phi$ was empirically fitted by Vieira and Brook [1984] to an exponential form, i.e.

$$
\phi \propto \exp (a P) \quad \text { Eqn. } 4.2
$$

where $a$ is a constant that depends on the dihedral angle and $P$ is the porosity. This relationship was experimentally verified by Rahaman and De Jonghe, and for the present $\mathrm{ZnO}$ system $a$ equals 5 [Rahaman and De Jonghe 1987b].

Strictly, the interpore spacing, $x$, should only included pores on the grain boundary, and is the most important parameter determining the densification strain rate. So far few, if any, systematic measurements of $x$ are known. In contrast, extensive data on grain size evolution are available, and their size is generally taken as the diffusion length of concern. It is important to recognize, however, that under various conditions the interpore spacing, $x$, can differ significantly from the grain size. It can be envisioned that local densification must first lead to dense interpore domains which can in turn support grain growth. A low grain growth rate causes the grain size to lag behind the increase in the mean pore spacing; a fast grain growth rate will lead either to a grain size equivalent to the pore spacing or a grain size larger than the pore spacing. In the first case, the pores effectively impede grain growth; in the latter, pore break-away occurs and the pores are left within the grains. These problems have been studied exhaustively (although perhaps not conclusively) in the sintering literature and will not 
be addressed here (see section 2.3). During sintering most pores in $\mathrm{ZnO}$ remain on the grain boundary and are fairly evenly distributed within the sample. Therefore the model considered here is one in which the interpore spacing and the grain spacing remain strictly proportional.

Measurements of grain growth in porous systems have indicated that (see section 2.3)

$$
G^{m}=G_{o}^{m}+g(T) t
$$

where $G_{0}$ is the starting grain size at $t=t_{0}$, and $m$ depends on the system. The grain growth rate coefficient $g(T)$ is generally written as

$$
g(T)=g_{\circ} \exp \left(-\frac{Q_{g}}{k T}\right)
$$

$\mathrm{go}_{\mathrm{o}}$ is a constant and $\mathrm{Q}_{\mathrm{g}}$ is the activation energy for grain growth. The exponents $\mathrm{m}$ and $\mathrm{n}$ for grain growth and densification in Eqns. 4.3 and 4.1, respectively do not have to be equivalent. For $\mathrm{ZnO} n$ is equal to 2 for volume diffusion, and $\mathrm{m}$ is approximately equal to 3 [Rahaman and De Jonghe 1987b].

Similarly, the coarsening function can be written as

$$
x^{m}=x_{0}^{m}+C(T) t
$$

Eqn. 4.5

where $x_{0}$, is the starting mean pore spacing at $t=t_{0}$, and $C(T)$ is the coarsening rate coefficient 


$$
C(T)=C_{o} \exp \left(-\frac{Q_{c}}{k T}\right)
$$

$C_{o}$ is a constant, and $Q_{c}$ is the activation energy associated with coarsening.

For non-isothermal conditions, where the temperature changes with time, Eqn. 4.5 can be written as

$$
x^{m}(T, t)=x_{o}^{m}+C_{0} \int_{t_{0}}^{t} \exp \left(-\frac{Q_{c}}{k T}\right) d t
$$

In constant heating rate experiments, for which $T=T_{0}+\alpha t$, with $\alpha$ as the heating rate, the increment of time, $d t$, is related to the temperature increment, $d T$ through $\alpha$, i. e.

$$
d t=\frac{d T}{\alpha}
$$

Then Eqn. 4.7 becomes

$$
x^{m}(T)=x_{0}^{m}+\frac{C_{0}}{\alpha} \int_{T_{0}}^{T} \exp \left(-\frac{Q_{c}}{k T}\right) d T
$$

For constant heating rates, time and temperature are proportional, leading to the expectation that the final grain size to the $\mathrm{m}$ power, proportional to $\mathrm{x}^{\mathrm{m}}$, should be inversely proportional to the heating rate, $\alpha$. Figure 4.5 shows that the cube of the final grain sizes indeed depends inversely on the heating rates used (correlation coefficient $=0.995$ ), supporting the validity of Eqn. 4.9. 
In any system the mean pore spacing will increase when a pore disappears for any reason. The removal of a pore can be accomplished by processes involving either densifying mechanisms or non-densifying mechanisms. These two processes are distinct, and are likely to have different activation energies and different relative importances. Processes with low activation energies will dominate at the low temperatures and processes with high activation energies will be relatively more important at the higher temperatures. Since constant heating rate experiments sample a wide temperature range the coarsening function, $x^{m}$, will be more appropriately described by including two temperature-dependent components: one relating to densifying processes, and the other relating to non-densifying processes. As a first approach to a function incorporating these two components, Eqn. 4.9 is rewritten as

$x^{m}(T)=x_{0}^{m}+\frac{A_{0}}{\alpha} \int_{T_{0}}^{T} \exp \left(-\frac{Q_{\alpha}}{k T}\right) d T+\frac{B_{0}}{\alpha} \int_{T_{0}}^{T} \exp \left(-\frac{Q_{n d}}{k T}\right) d T$

Eqn. 4.10

$\mathrm{x}_{\mathrm{O}}$ is the starting mean pore separation; the second term is associated with densifying mechanisms, where $A_{o}$ is a constant and $Q_{d}$ is an activation energy descriptive of densifying mechanisms; the third term is associated with non-densifying mechanisms, where $\mathrm{B}_{\mathrm{O}}$ is a constant and $\mathrm{Q}_{n d}$ is an activation energy descriptive of non-densifying mechanisms. In principle, more than one term of this type would need to be included; for the present, however, only one dominant term for each type of mechanisms will be considered.

The form of Eqn. 4.10 can be rationalized if one assumes, by analogy to grain growth, that it results from an additive expression for the coarsening rate;

$$
\frac{d x}{d t}=\frac{A^{\prime}}{x^{p}}+\frac{B^{\prime}}{x^{q}}
$$


where $A^{\prime}$ and $B^{\prime}$ are the temperature dependent rate constants of the form $A_{0} \exp (-Q / k T)$ describing the separate densifying and non-densifying processes. If one takes $p$ to equal $q$ then the form of Eqn. 4.10 follows; if $p$ does not equal $q$, as would have to be expected in the general case, a more complicated expression would result. For now $\mathrm{p}=\mathrm{q}$ is assumed.

One merit of Eqn. 4.10, is that it brings out clearly the dependence of the coarsening on the two different classes of transport processes. The constants $A_{O}$ and $B_{O}$ will determine the relative importance of the two processes. Typically, it should be expected that an activation energy for non-densifying coarsening, $Q_{\text {nd }}$, would be significantly lower than $Q_{d}$, when it involves surface transport in a continuous pore network. A general assertion about activation energies can, however, not be made, as actual values are likely to be system specific.

It is conceivable and likely that in addition to temperature the two components of coarsening are dependent on the sintered density of the material such that only certain types of mechanisms are important for a particular microstructure. For example, at the lower densities where the pores are open and connected, surface diffusion can proceed. However, at higher densities where the pores become isolated surface diffusion or vapor transport can no longer be active and other mechanisms must dominate. The various coarsening components will again have different activation energies but the associated prefactors will depend on the sintered density. In the present study the sintered density is too ciosely related to the temperature to determine a density dependence. To measure the density dependencies of the coarsening components studies with a wider density to temperature range are necessary.

For constant heating rates the densification strain rate equation, Eqn. 4.1, is now rewritten as 


$$
\dot{\epsilon}_{d}=\frac{K D(T) \phi^{\frac{n+1}{2}}}{(x(T))^{n} k T}
$$

where $K=H \Sigma$ and is constant, $(x(T))^{n}$ is given by Eqn. 4.10, and $D(T)$ is the diffusion coefficient for the densifying transport mechanism:

$$
D(T)=D_{0} \exp \left(-\frac{Q_{d}}{K T}\right)
$$

where $D_{o}$ is a constant and $Q_{d}$ is the activation energy associated with densification.

\subsubsection{Limiting Cases of No Coarsening and Extreme Coarsening}

The limiting caser, of extreme coarsening and of no coarsening, can be examined using Eqns. 4.10 and 4.12.

First, consider the case where little or no coarsening is observed, $x=x_{0}$; Eqn. 4.12 becomes

$$
\dot{\epsilon}_{d}=\frac{1}{3 \rho} \frac{d \rho}{d t}=K D_{0} \exp \left(-\frac{Q_{d}}{k T}\right) \frac{\phi^{\frac{n+1}{2}}}{x_{0}^{n} k T} \quad \text { Eqn. } 4.14
$$

Separating the variables and using the constant heating rate conditions, $\mathrm{T}=\mathrm{To}+\alpha \mathrm{t}$, Eqn. 4.14 is rewritten as

$$
\int_{\rho 0}^{\rho}\left(\frac{1}{\phi^{\frac{n \cdot 1}{2}}}\right)\left(\frac{1}{3 \rho}\right) d \rho=F(\rho)=\frac{K}{\alpha x_{0}^{n}} \int_{T_{0}}^{T D_{0} \exp \left(-\frac{Q_{d}}{k T}\right)} \frac{k T}{k T} d T \text { Eqn. } 4.15
$$


where the right side depends on the inverse of the heating rate and some function, $\mathrm{F}(\mathrm{T})$, that is independent of the heating rate. Due to the dependence of $\phi$ on density, $F(\rho)$ is not a simple function of density, but $F(\rho)$ does increase monotonically with density. This means that at a particular temperature, $T$, the density of the sample with the slower heating rate will be higher than for the sample with a faster heating rate. To evaluate the dependence of the sintered density on the heating rate, $F(\rho)$ can be fitted to a function that depends on the incremental density and the stress intensification factor, as follows:

$$
F(\rho)=\left(\frac{\Delta \rho}{\phi^{\frac{n+1}{2}}}\right)^{\lambda}
$$

By numerical trial and error, $\lambda$ is found to be approximately 0.7 for the density range between 0.5 and 1.0. Substituting Eqn. 4.16 into Eqn. 4.15 the incremental density becomes

$$
\Delta \rho=\left(\frac{1}{\alpha}\right)^{\frac{1}{\lambda}}(\phi)^{\frac{n+1}{2}}(F(T))^{\frac{1}{\lambda}}
$$

Under conditions of no coarsening the incremental density reached at a particular temperature is then proportional to the heating rate to the -1.4 power: the slower heating rate will attain a higher density.

In the other extreme, where significant coarsening has occurred:

$$
x_{0}^{m} \ll A_{0} \int \exp \left(-\frac{Q_{d}}{k T}\right) d t+B_{0} \int \exp \left(-\frac{Q_{n d}}{k T}\right) d t
$$


Now Eqn. 4.12 can be written as

$$
\frac{\dot{\epsilon}}{\phi^{\frac{n+1}{2}}}=\frac{\alpha^{\frac{n}{m}} K D_{0} \exp \left(-\frac{Q_{d}}{k T}\right)}{k T\left(A_{0} \int \exp \left(-\frac{Q_{d}}{k T}\right) d T+B_{0} \int \exp \left(-\frac{Q_{n d}}{k T}\right) d T\right)^{\frac{n}{m}} \text { Eqn. } 4.19}
$$

and separating the variables and integrating, Eqn. 4.19 now becomes

$$
F(\rho)=\alpha^{\frac{n}{m}-1} G(T)
$$

where $G(T)$ is a function independent of the heating rate and sintered density. Substituting again Eqn. 4.16 for $F(\rho)$, Eqn. 4.20 yields

$$
\Delta \rho=\left(\alpha^{\frac{n}{m}-1}\right)^{\frac{1}{\lambda}}(\phi)^{\frac{n+1}{2}}(G(T))^{\frac{1}{\lambda}} \quad \text { Eqn. } 4.21
$$

It is immediately clear that when the densification exponent, $n$, and the grain growth exponent, $m$, are the same the density at a given temperature is independent of the heating rate. For $\mathrm{ZnO} n=2$ and $m=3$, with $\lambda=0.7$ the density reached at a particular ternperature will be proportional to approximately $\alpha^{-0.5}$, so it has a relatively weak dependency on the heating rate. Such a dependence is, at least, qualitatively evident in Fig. 4.1.

It is interesting to contrast the expected consequences of heating rate on systems that coarsen little (e.g. monosize particles) with those that coarsen significantly. As evident in Eqn. 4.15, when coarsening is absent or minimal then at a specific temperature, the incremental densification strain, $\Delta \epsilon$, should increase significantly when the heating rate decreases. This behavior has, in fact, been observed for a near monomodal system of $\mathrm{TiO}_{2}$ by Barringer et al [1984]. Figure 4.6 shows some of the 
data obtained by Barringer et al, at $1160^{\circ} \mathrm{C}$ replotted to reflect the incremental shrinkage as a function of heating rate. Even at the highest shrinkage $\left(\Delta 1 / 1_{0}\right)=0.15$ or $\log \left(\Delta 1 / 1_{0}\right)=0.82$, with a corresponding relative density is about 0.9$)$ little coarsening was reported. As Fig. 4.6 shows the experimental results are in good agreement with $\left(\Delta 1 / 1_{0}\right) \propto(\Delta \rho) \propto\left(\alpha^{-1.4}\right)$ predicted by Eqn. 4.17.

For the present $\mathrm{ZnO}$ system, in which coarsening is significant, $\dot{\epsilon}_{d} /\left(\phi^{3 / 2}\right)$ should be proportional to the heating rate to some power, as follows from Eqn. 4.19. It is shown in Fig. 4.7 that $\alpha^{2 / 3}$ fits the observation reasonably well. For higher heating rates, where coarsening is less pronounced, it is expected that some deviation from this relationship will become apparent.

A striking feature of the data is that the temperature derivative of the volumetric strain, $(1 / \rho) \mathrm{d} \rho / \mathrm{dT}$, plotted as a function of sintered density, $\rho$, fits into a relatively narrow band for the wide range of heating rates used (Fig. 4.2). Similar data have been obtained recently by Lange [1989] for $\mathrm{Al}_{2} \mathrm{O}_{3}$ powders. The $\mathrm{Al}_{2} \mathrm{O}_{3}$ data also showed that the shrinkage strain rate increased to a maximum at $\rho=0.77$ and then decreased, from which Lange concluded that the sintering kinetics controlled densification below $\rho=0.77$ after which coarsening kinetics dominated. As seen from Fig. 4.2, the present data for $\mathrm{ZnO}$ do not show a sharp maximum at $\rho=0.77$; instead a relatively constant, plateau region between $\rho$ values of 0.60 and 0.80 is observed.

The relationship between $\mathrm{d} \epsilon / \mathrm{dT}$ and $\Delta \rho$ follows from Eqns. 4.8, 4.19, and 4.21:

$$
\left(\frac{\Delta \rho}{\frac{d \epsilon}{d T}}\right) \propto \alpha^{\left(\frac{n}{m}-1\right)\left(\frac{1}{\lambda}-1\right)}
$$


for $\mathrm{ZnO}(\mathrm{n}=2, \mathrm{~m}=3$, and $\lambda=0.7)$, Eqn. 4.22 predicts a slight dependence on the heating rate $\left(\sim \alpha^{-0.1}\right)$ for a plot of $\mathrm{d} \in / \mathrm{dT}$ versus $\Delta \rho$ (or $\rho$ ).

This behavior is seen in Fig. 4.2 where $\mathrm{d} \epsilon / \mathrm{dT}$ as a function of density is plotted for all the different heating rates used: all data fall within a narrow band. Figure 4.2 actually shows less dependence on $\alpha$ than is consistent with $n=2$ and $m=3$. One possible explanation is that $\mathbf{n}$ and $\mathrm{m}$ were not determined with sufficient accuracy from the available experimental data and differ less than for the case of volume diffusion assumed here.

\subsubsection{Numerical Calculations of the Coarsening Function. $x^{m}$}

The average interpore spacing, $x$, of samples from various heating rates can be extracted from the densification strain rate data with Eqn. 4.12, i.e.

$$
x^{2}=\frac{K^{\prime} \phi^{\frac{3}{2}}}{k T \dot{\epsilon}_{d}} \exp \left(-\frac{Q_{d}}{k T}\right)
$$

With the data from Figs. 4.1 and 4.4, Eqn. 4.2, and an activation energy for densification taken to be $50 \mathrm{kcal} / \mathrm{mole}^{*}$, the behavior of $\mathrm{x}$ as a function of temperature can be determined. $\log \left(x^{2}\right)$ of the samples from various heating rates versus temperature is plotted in Fig. 4.8. As seen from the figure the mean interpore spacing increases for all conditions, and at a given temperature the interpore spacing resulting from the slower heating rate is larger than that from of the faster heating rate. Both

\footnotetext{
* The activation energy used here is lower than most values cited by Gupta and Coble [1968] (40 - $80 \mathrm{kcal} / \mathrm{mole})$. The higher activation energies would predict final grain sizes that are too large to reflect the present data.
} 
these behaviors are commonly observed. But a dependence of the interpore spacing on heating rate is evident from the onset of densification. It will be shown that two different coarsening processes, one with a high and one with a low activation energy, are necessary to account for this behavior.

The complexity of the expressions for the dependence of the densification strain rate, $\dot{\epsilon}_{d}$, on temperature, even in the experimental limits of low and high temperature, precludes a straightforward extraction of meaningful activation energies from the sintering data in the general case. In the case of negligible coarsening, as expected with large or monosize particles at high heating rates, it should be observed that $\dot{\epsilon}_{d}$ at a given temperature is nearly independent of the heating rate. If such is the case, then the activation energy for diffusional transport can be obtained with some confidence from the low-temperature region of the constant heating rate data. In other cases the qualitative features of the coarsening versus temperature can be reproduced by numerically integrating Eqn. 4.10 and adjusting the values of the various constants.

Assuming only one coarsening process to be active - the one associated with densifying processes and characterized by $\mathrm{Q}_{d}$ - Eqn. 4.5 will predict coarsening behavior as shown qualitatively in Fig. 4.9a. This plot was generated by using $Q_{d}=50 \mathrm{kcal} / \mathrm{mole}$, with values for the prefactor ${ }^{*}$ and $x_{0}$ such that $x$ at $1100^{\circ} \mathrm{C}$ is equal to $\sim 60 \mathrm{x}_{0}$ for the heating rate of $5^{\circ} \mathrm{C} / \mathrm{min}$ as suggested by the grain sizes measured on polished sections. Here, the calculated interpore spacings at heating rates of $15^{\circ} \mathrm{C} / \mathrm{min}, 5^{\circ} \mathrm{C} / \mathrm{min}$, and $2{ }^{\circ} \mathrm{C} / \mathrm{min}$ are initially independent of the heating rate, $\alpha$, and remain fairly constant as long as little coarsening has occurred. As temperature increases and coarsening starts to become significant, the mean interpore spacing increases rapidly. Low

$A_{0}=1.0 \times 10^{0}$
$x_{0}=4.0 \times 10^{-4}$ 
temperature separation between the various curves will occur if the activation energy is reduced. Then, however, at higher temperatures the spacing does not increase rapidly enough to reflect the data. The calculated values of $x^{2}$ versus temperature for 30 $\mathrm{kcal} / \mathrm{mole}$ are shown in Fig. $4.9 \mathrm{~b}$.

The major feature of the $\mathrm{ZnO}$ data, Fig. 4.8, is that the mean interpore spacings are proportional to the heating rate from the onset of observable densification. This behavior cannot be reproduced by using only one activation energy for coarsening.

Two components, a low temperature coarsening process, such as associated with a non-densifying mechanism with an activation energy, $Q_{n d}$, and densifying mechanisms with activation energy, $Q_{d}$, must therefore be included in the calculation to conform, even qualitatively, to the experimental results. The calculated behavior ${ }^{*}$ from Eqn. 4.10, shown in Fig. 4.10 and approximating well the observed coarsening behavior of $\mathrm{ZnO}$, was generated using $\mathrm{Q}_{\mathrm{nd}}=15 \mathrm{kcal} / \mathrm{mole}$ and $\mathrm{Q}_{\mathrm{d}}=50 \mathrm{kcal} / \mathrm{mole}$, with the prefactors and $x_{0}$ chosen such that $x_{\text {at }} 550^{\circ} \mathrm{C}$ is twice $x_{0}$ and $x$ at $1100^{\circ} \mathrm{C}$ is $\sim 60 x_{0}$ for the heating rate of $5^{\circ} \mathrm{C} / \mathrm{min}$.

It can therefore be concluded that the description of the coarsening process in $\mathrm{ZnO}$ under constant heating rates is consistent with a coarsening process, i.e., an evolution of the mean interpore spacing, that has two components: a low temperature one of low activation energy, operating while the densification strain rate is very low, and thus associated with non-densifying processes, and a coarsening mechanism operating at high temperatures with an activation energy comparable to that of

$\begin{aligned} A_{0} & =1.0 \times 10^{0} \\ B_{0} & =5.6 \times 10^{-9} \\ x_{O} & =4.0 \times 10^{-4}\end{aligned}$ 
densification and therefore associated with densifying mechanisms. It is the latter that causes the densification strain rate under constant heating rate conditions to decrease before the final density is reached.

While the experimental examinations are limited here to $\mathrm{ZnO}$, it is plausible to suppose that similar processes of coarsening, characterizable by contributions of densifying and non-densifying processes, may be recognized in most real non-isothermal sintering of ceramic powder compacts.

\subsection{Summary}

The significance of coarsening for sintering is best revealed when the process is defined as an increase in the mean pore separation. Analysis of the constant heating rate conditions can then be performed using a modified isothermal densification strain rate equation to account for the changes in the pore spacing as a function of time and temperature. The modification consists in recognizing that for a sample with a distribution of pore sizes there are contributions of densifying and non-densifying processes to coarsening.

The sintering of $\mathrm{ZnO}$ at various heating rates is consistent with the model in which coarsening is significant from the onset of sintering. The densification strain rate behavior with respect to temperature is consistent with a coarsening process characterized by two types of activated process, one attributed to densifying processes at higher temperatures and the other to non-densifying processes at lower temperatures. 
For sintering without coarsening, where $x(T, t)=x_{0}$, the modified rate equation predicts that the incremental strain, $\Delta \epsilon$, at a given temperature decreases as the heating rate increases. This result is consistent with the sintering behavior of monosized particles.

For significant coarsening, where $x>>x_{0}$, the modified densification strain rate, i.e. $\dot{\epsilon}_{d} /\left(\phi^{3 / 2}\right)$ at a given temperature is predicted to be proportional to the heating rate, $\alpha^{n / m}$, while the incremental density reached at a given temperature is independent of the heating rate when the densification and grain growth exponents are equal and weakly dependent on the heating rate when the two exponents are not equivalent.

It is shown that a heating-rate independent relation between $\mathrm{d} \epsilon / \mathrm{dT}$ and $\Delta \rho$ follows directly from constant heating rate conditions when the densification and grain growth exponents are the same, since under these conditions both $\mathrm{d} \epsilon / \mathrm{dT}$ and therefore $\Delta \rho$ are independent of the heating rate, $\alpha$. When the exponents differ, $d \in / d T$ and $\Delta \rho$ become weakly dependent on the heating rate. 


\section{EFFECT OF COARSENING ON SINTERING AND MICROSTRUCTURE}

\section{Abstract}

$\mathrm{MgO}$ and $\mathrm{Al}_{2} \mathrm{O}_{3}$ compacts were sintered by two types of process: a conventional isothermal sintering, and a two-step sintering consisting of an initial low temperature pre-coarsening treatment before a conventional isothermal sintering. The final microstructure from the two-step sintering is more uniform and finer than that of compacts sintered conventionally.

The differences between two-step and conventional processing are clarified by experiments in pre-coarsened and as-received $\mathrm{ZnO}$ powders. These compacts were pre-coarsened at $450^{\circ} \mathrm{C}$ for 90 hours with virtually no increase in the overall density. The resulting grain size was 1.7 times the starting one, but the standard deviation of the pre-coarsened powder size distribution was narrower than that of the as-received powder. The sintering stress of the pre-coarsened $\mathrm{ZnO}$ is approximately 0.8 that of the as-received one. A computational model has been used with two components of coarsening to describe the differences in evolution of pore spacing between the pre-coarsened and the as-received system.

The benefit of two-step sintering is attributed to the increase in uniformity resulting from pre-coarsening. The increased uniformity decreases sintering damage and allows the system to stay in the open porosity state longer, delaying or inhibiting additional coarsening (grain growth) during the final stage of densification. Two-step sintering is especially useful for non-uniform powder systems with a wide size distribution and is a simple and convenient method of making more uniform ceramic bodies without resorting to specialized powders or complicated heat schedules. 


\subsection{Introduction}

Agglomeration is a problem common to most ceramics powders, and is well known to increase with decreasing particle size [Rhodes 1981]. Green bodies formed from such powders contain regions of non-uniform packing, and these lead to differential densification and sintering damage. The powder compact is particularly susceptible to the development of such sintering damage in the early stages of densification since the interparticle necks are then small and can rupture easily. Direct observations of the development of sintering damage were reported in the work of Weiser ${ }_{6}$ and De Jonghe [1986] on planar arrangements of uniform copper spheres. Damage from differential densification was most serious in the earliest stages of densification.

While some sintering damage is virtually unavoidable, efforts to produce more homogeneous initial powder compacts can reduce the problem. A number of researchers have addressed the issue of initial heterogeneity. Yan et al [1983] demonstrated that when the size distribution of the powders was narrowed, the relative densities could be increased from 0.9 to 0.99 . Carpay [1977] found that a wide pore size distribution increases the tendency for exaggerated grain growth. Carlstrom et al [1983] observed that for alpha-SiC, heterogeneities in the green body tend to persist and inhibit sintering to high final densities. Kaysser and Lenhart [1981] showed that porosity was reduced in sintered $\mathrm{ZnO}$ agglomerates when the agglomerates were presintered to promote neck formation. Numerous other workers have tried to manipulate microstructures by controlling the sintering heat schedule. "Ratio control sintering" [Brook 1982, Pickup et al 1985] is a process by which the relative rates of coarsening and densification are determined by the choice of the sintering temperature. For example, in the case of $\mathrm{Al}_{2} \mathrm{O}_{3}$ the activation energy for densification is higher than that for grain growth, and sintering at high temperatures is therefore indicated. For $\mathrm{MgO}$, however, the relationship between activation energies for grain growth and 
densification is uncertain, and it is therefore more difficult to specify an optimum sintering temperature. A corollary of "ratio controlled sintering" is "ultra-rapid firing" [Johnson 1984], which can produce dense material with fine grain size by minimizing the time spent at temperatures where grain growth is rapid compared to densification. "Rate-controlled sintering" [Palmour and Johnson 1967] in which attempts are made to keep the densification rate constant by adjusting the temperature, in some cases has also been reported to have beneficial effects on the microstructure. These methods work best for non-agglomerated powder compacts; for agglomerated systems the benefits are less clear, and additional processing steps must be developed that could remedy, at least in part, the common heterogeneities produced during powder consolidation.

The approach taken in the present work has been to pre-coarsen the initial powder compact at some low temperature where little or no densification occurs. In addition to strengthening the necks between particles, thereby decreasing the risk of sintering damage development from differential densification, the powder compacts were found to evolve towards a microstructure that is more uniform than that of the initial powder. The combination of these two effects is exploited in a two-step sintering heat schedule that incorporates such pre-coarsening before firing at higher temperatures. The results of this two-step sintering are contrasted with those of a conventional firing schedule that brings the compacts directly to the final firing temperature, the single-step sintering. The materials examined initially were magnesium oxide and aluminum oxide, while more detailed information on the consequences of pre-coarsening was derived from studies on zinc oxide. 


\subsection{Experimental Procedure}

\subsubsection{Magnesium Oxide}

$\mathrm{MgO}^{*}, "$ powder (diameter $6 \mathrm{~mm}$ by $5 \mathrm{~mm}$ ) was compacted in a die to an initial relative density of $0.40 \pm 0.01$. This powder, which was also used in earlier studies [Lin et al 1987], is shown in Fig. 5.1; it has a narrow size distribution but is agglomerated.

Conventional one-step sintering was accomplished isothermally at $1600^{\circ} \mathrm{C}$ for 1 hour. The sampls was first heated to $850^{\circ} \mathrm{C}$ at $10^{\circ} \mathrm{C} / \mathrm{min}$ and then heated to $1600^{\circ} \mathrm{C}$ at approximately $75^{\circ} \mathrm{C} / \mathrm{min}$. For two-step sintering the same $\mathrm{MgO}$ pellets were initially pre-coarsened at $1250^{\circ} \mathrm{C}$ for 48 hours and then sintered at $1600^{\circ} \mathrm{C}$ for one hour. The relative density after the $1250^{\circ} \mathrm{C}$ heat treatment was $0.80 \pm 0.01$ and the final density for both single-step and two-step sintering was $0.93 \pm 0.01$. Schematics of the temperature schedule for the two types of sintering are shown in Fig. 5.2.

Fracture surfaces were examined by scanning electron microscopy (SEM). The average grain size was measured from polished sections. In addition, compacts with the same starting density were sintered isothermally at either $1500^{\circ} \mathrm{C}$ or $1250^{\circ} \mathrm{C}$. Heating schedules were selected such that the samples could be compared at constant sintered densities. Fracture surfaces of samples at relative densities of $0.6,0.7$, and 0.8 were examined by SEM.

- Two-step experiments on $\mathrm{MgO}$ and $\mathrm{Al}_{2} \mathrm{O}_{3}$ were performed by $\mathrm{M}$. Lin.

* Reagent Grade, J. T. Baker Chemical Co., Philipsburg, N. J. 


\subsubsection{Aluminum Oxide}

Procedures similar to those for $\mathrm{MgO}$ were used for $\mathrm{Al}_{2} \mathrm{O}_{3}{ }^{*}$. For one step sintering, compacts were sintered at $1600^{\circ} \mathrm{C}$ for 1 hour. The two-step sintering schedule included a pre-coarsening treatment at $1300^{\circ} \mathrm{C}$ for 24 hours before sintering at $1600^{\circ} \mathrm{C}$ for 1 hour. The final relative density for both single and two-step sintering was 0.95 \pm 0.01 .

\subsubsection{Zinc Oxide}

$\mathrm{ZnO}^{* *}$ powder $(6 \mathrm{~mm}$ in diameter by $5 \mathrm{~mm})$ was uniaxially compacted in a tungsten carbide die, at $\sim 10 \mathrm{MPa}$ to an initial relative density of $0.49 \pm 0.01$. The same powder was used for experiments described in sections 3 and 4. Pre-coarsening was accomplished by heating the compacts at $450^{\circ} \mathrm{C}$ for 90 hours, in air. The density of the pellets increased to $0.50 \pm 0.01$ during pre-coarsening. The fracture surfaces of the as-received and pre-coarsened powder compacts are shown in Figs. 5.3a and 5.3b, respectively,

Both the as-received and pre-coarsened compacts were then sintered in air, under identical conditions, in a loading dilatometer [De Jonghe and Rahaman 1984a]. Sintering was completed with zero load or with a uniaxial load of 7 Newton, at a constant heating rate $(\mathrm{CHR})$ of $8^{\circ} \mathrm{C} / \mathrm{min}$. The load of 7 Newton, corresponding to an initial stress on the compact of $0.2 \mathrm{MPa}$, was applied at $520^{\circ} \mathrm{C}$ when shrinkage just commenced. The experiment was stopped at $1200^{\circ} \mathrm{C}$. The densification was nearly complete by $1000^{\circ} \mathrm{C}$. The mass and dimensions of the samples were measured before and after sintering, and the final densities were verified using Archimedes' method. In

- Alcoa Chemicals, Pittsburg, Pa.

** Reagent Grade, Mallinckrodt Inc., Paris, Kentucky 
a separate set of experiments, sintering with load was terminated at temperatures between $520^{\circ} \mathrm{C}$ and $1200^{\circ} \mathrm{C}$, and the dimensions of these compacts were measured using a micrometer. Fracture surfaces and polished sections were examined with the SEM.

\subsection{Results}

\subsubsection{Sintering of Magnesium Oxide and Aluminum Oxide}

Microstructures after a single isothermal heating at $1500^{\circ} \mathrm{C}$ or at $1250^{\circ} \mathrm{C}$, at sintered densities of 0.6, 0.7, and 0.8 are compared in Figs. 5.4, 5.5, and 5.6. At constant density, in each case the grain size of the compact sintered at $1250^{\circ} \mathrm{C}$ is larger than that of the compacts sintered at $1500^{\circ} \mathrm{C}$. The difference in grain sizes is more dramatic as density increase.

Microstructures of $\mathrm{MgO}$ and $\mathrm{Al}_{2} \mathrm{O}_{3}$ after conventional single-step sintering and after two-step sintering are shown in Figs. 5.7 and 5.8, respectively. For $\mathrm{MgO}$ and $\mathrm{Al}_{2}, \mathrm{O}_{3}$ both single and two-step sintering processes led to the same final density, but the microstricture from the two-step process appears more uniform than that from conventional sintering. The average grain size of $\mathrm{MgO}$ after each step, plotted as a function of density, is shown in Fig. 5... The grain size at 0.8 density for sintering at $1600^{\circ} \mathrm{C}$ was not obtained but, extrapolating from the results of $1250^{\circ} \mathrm{C}$ and $1500^{\circ} \mathrm{C}$ single-step sintering, the grain size should be smaller than that of the compact sintered at $1250^{\circ} \mathrm{C}$ to a relative density of 0.8 .

\subsubsection{As-received versus Pre-coarsened Zinc Oxide}

The microstructures of as-received and of pre-coarsened $\mathrm{ZnO}$ are shown in Figs. 5.3a and 5.3b. For the as-received $\mathrm{ZnO}, 75$ particles were measured and their average size was $0.09 \pm 0.07 \mu \mathrm{m}$; for the pre-coarsened sample 50 paı ticles were measured and 
the average particle size increased to $0.15 \mu \mathrm{m}$ but the standard deviation of the particle size distribution had actually decreased measurably to $\pm 0.06 \mu \mathrm{m}$. This decrease in the standard deviation, not only in relative but also in absolute magnitude, reflects the microstructural homogenization that accompanies pre-coarsening.

Figure 5.10 shows the axial true strain, $\epsilon_{z}$, versus temperature for the as-received and for the pre-coarsened $\mathrm{ZnO}$ samples sintered at a constant heating rate of $8^{\circ} \mathrm{C} / \mathrm{min}$, under zero applied stress and under an initial applied stress of $0.2 \mathrm{MPa}$. Each curve is an average of two runs under identical conditions; the reproducibility is $\pm 1 \%$. Results for the axial true strain, $\epsilon_{z}$, versus the radial true strain, $\epsilon_{r}$, for as-received and pre-coarsened $\mathrm{ZnO}$ :amples were determined by quenching the sample from temperatures between 520 and $1200^{\circ} \mathrm{C}$. These results are shown in Fig. 5.11; each datum point was obtained by using a different sample. The data in Fig. 5.11 have been converted to constant-stress conditions, corresponding to the initial stress of $0.2 \mathrm{MPa}$. To obtain data under constant-stress conditions the constant load data require a correction for dimensional changes due to densification (see section 3.4). The slopes of these lines, i.e. $\epsilon_{z} / \epsilon_{r}$, for as-received and for pre-coarsened $\mathrm{ZnO}$ are 1.207 and 1.270 , respectively. The shrinkage of the sample sintered under no load is seen to be almost isotropic.

From Figs. 5.10 and 5.11 the sintered densities of as-received and the pre-coarsened samples are calculated. In Fig. 5.12 the densities of the pre-coarsened $\mathrm{ZnO}$ sintered under zero load or with an applied load are plotted as a function of temperature. Within the limits of experimental error the applied load has no effect on the densification. The deformation of the sample under the action of the applied stress of $0.2 \mathrm{MPa}$ is therefore constant volume creep. Both the as-received $\mathrm{ZnO}$ and 
pre-coarsened $\mathrm{ZnO}$ reached the same final density of 0.99 . Also, as seen in Fig. 5.13, in the intermediate temperature range the density of the as-received $\mathrm{ZnO}$ is higher than that of the pre-coarsened $\mathrm{ZnO}$.

For a body loaded uniaxially and deforming uniformly it is readily shown that the linear densification strain, $\epsilon_{d}$ is

$$
3 \epsilon_{d}=\epsilon_{z}+2 \epsilon_{r}
$$

where $\epsilon_{d}$ is the axial true strain and $\epsilon_{r}$ is the radial true strain. Correspondingly, the total axial strain, $\epsilon_{z}$, is:

$$
\epsilon_{z}=\epsilon_{c}+\epsilon_{d}
$$

Eqn. 5.2

where $\epsilon_{c}$ is the creep strain. It follows that

$$
\epsilon_{c}=\frac{2}{3}\left(\epsilon_{z}-\epsilon_{r}\right)
$$

With Eqns. 5.1 - 5.3 the densification and creep strains and strain rates are found. The densification strain rates and the creep strain rates of the two types of $\mathrm{ZnO}$ are plotted versus temperature, in Figs. 5.14 and 5.15. In both cases the as-received $\mathrm{ZnO}$ starts at a higher densification and creep strain rate than the pre-coarsened sample, but later the opposite is observed. 


\subsection{Discussion}

The discussion has been divided into four sections. First, the effect of pre-coarsening on the sintering stress, i.e. the driving force for free sintering, is considered. Next, the effect of pre-coarsening on the evolution of the center-to-center pore spacings, $x(t, T)$ is described for the two types of samples, based on the creep and densification data. Then, the modelling of $x(t, T)$ is developed, and calculated and experimentally based values of $x(t, T)$ are compared. Finally, the application of two-step sintering, i.e. pre-coarsening of powder compacts prior to densification, is discussed.

\subsubsection{Effect of Pre-coarsening on the Sintering Stress}

The results of two-step and of single-step sintering under conditions described here should follow from an appropriate sintering and microstructural evolution model and its constitutive equations. Here the results will be interpreted in the context of an expression, developed in sections 3.5.2 and 4.4.1, for the densification and creep strain rates as well as for the microstructural evolution.

The instantaneous isothermal densification strain rate is (see Eqn. 4.12)

$$
\dot{\epsilon}_{d}=H D_{0} \sum \exp \left(-\frac{Q_{d}}{k T}\right) \frac{\phi^{\frac{n+1}{2}}}{k T x^{n}} \quad \text { Eqn.5.4 }
$$

where $H$ is a constant, $\Sigma$ is the sintering stress (which drives free sintering), $D_{0}$ is the temperature insensitive component of the diffusion coefficient, $Q_{d}$ is the activation energy for densification, $k$ is Boltzman's constant, $T$ is the absolute temperature, $\phi$ is the stress intensification factor [Beere 1975a\&b], $x$ is the center-to-center pore spacing 
(often taken as the grain size, see sections 2.3 and 4.4.1), and $\mathrm{n}$ is the appropriate exponent for densification, $(n=2$ for volume diffusion and $n=3$ for grain boundary diffusion, $\mathrm{n}=2$ for $\mathrm{ZnO}$ [Rahaman and De Jonghe 1987b]).

Under conditions of a low applied uniaxial stress, which induces creep without increasing the overall density, the mechanisms for densification and creep are intimately related [Rahaman and De Jonghe 1984a]. As evideni in the plot of density versus temperature for $\mathrm{ZnO}$ sintered with or without an applied stress (Fig. 5.12) the resulting densities are identical. The creep strain rate, superimposed on the densification strain rate, is then:

$$
\dot{E}_{c}=K_{c} D_{o} \sigma \exp \left(-\frac{Q_{d}}{k T}\right) \frac{\phi^{\frac{n+1}{2}}}{k T x^{n}}
$$

where $\mathrm{K}_{\mathrm{c}}$ is a constant and $\sigma$ is the uniaxially applied stress.

The relative magnitude of free-sintering driving force for the systems can be obtained from the ratio of the densification strain rate over the creep strain rate

$$
\frac{\dot{\epsilon}_{d}}{\dot{\epsilon}_{c}} \propto\left(\frac{\Sigma}{\sigma}\right)
$$

From results of the constant-stress creep and densification the sintering stress of the pre-coarsened ${ }^{*}$ sample, $\Sigma_{c}$, is found to be about 0.8 of the sintering stress for the as-received sample, $\Sigma$.

"The extra subscript " $c$ " will denote the coarsened sample. 
As coarsening proceeds, for a self-similar system a reduction in the driving force should be expected proportional to the inverse of its scale factor. Here, the average initial particle size of the pre-coarsened sample is approximately 1.7 times larger than that of the as-received sample. If the sintering stress strictly scales to the inverse of the mean particle size then one would expect $\Sigma_{c}$ to be 0.59 of $\Sigma$. The change in the magnitude of $\Sigma$ is not as large here; the difference could be due to other structural terms that arise in systems that are not strictly self-similar, such as a decrease in the average pore coordination number [Kellet and Lange 1989" which could increase the sintering stress.

\subsubsection{Effect of Pre-coarsening on the Evolution of the Center-to-center Pore} Spacing, $x(t, T)$

As evident from the resulting microstructure of $\mathrm{MgO}$ and $\mathrm{Al}_{2} \mathrm{O}_{3}$ the two-step sintering process produced a finer and more uniform grain structure than conventional single-step sintering. Due to temperature limitation of the dilatometer, sintering data to full density were not obtained for $\mathrm{MgO}$ and $\mathrm{Al}_{2} \mathrm{O}_{3}$. However, since the behavior of as-received and pre-coarsened $\mathrm{ZnO}$ corresponds in many aspects to single-step and two-step sintering, the $\mathrm{ZnO}$ data can give insight into the origin of the microstructural differences following the two treatments for $\mathrm{MgO}$ and $\mathrm{Al}_{2} \mathrm{O}_{3}$.

While the two types of heat treatment for the $\mathrm{ZnO}$ contain the essential elements

of those for the $\mathrm{MgO}$ and $\mathrm{Al}_{2} \mathrm{O}_{3}$ system, there are important differences. First, a constant heating rate rather than isothermal sintering is used for the $\mathrm{ZnO}$ pellets. $\mathrm{CHR}$ allows for a more precise analysis of the data, since isothermal sintering necessarily contains a heat-up and is actually a less defined heat treatment. In addition, CHR 
conditions access a wider range of sintered densities. Third, during the pre-coarsening of $\mathrm{MgO}$ and $\mathrm{Al}_{2} \mathrm{O}_{3}$ compacts there was significant densification. In contrast during pre-coarsening of $\mathrm{ZnO}$ there was virtually no increase in density.

The relative differences in the evolution of the center-to-center pore spacing, $x(t, T)$, between the as-received and pre-coarsened samples can be extracted from the corresponding densification strain rates. The ratio of the densification strain rate of the as-received sample over the pre-coarsened sample at any temperature follows from Eqn. 5.1:

$$
\frac{\dot{\epsilon}_{c d}}{\dot{\epsilon}_{d}}=\left(\frac{\phi_{c}}{\phi}\right)^{\frac{n+1}{2}}\left(\frac{x}{x_{c}}\right)^{n}\left(\frac{\Sigma_{c}}{\Sigma}\right)
$$

With the experimental ratios of $\Sigma_{c} / \Sigma=0.8$ and $\dot{\epsilon}_{c d} / \dot{\epsilon}_{d}$, and when the stress intensification factors for the as-received and the pre-coarsened samples have been determined from the densities at that temperature, $\left(x / x_{c}\right)^{n}$ can be found from Eqn. 5.7. Since $\phi$ is proportional to $\exp (a \mathrm{P})$ [Vieira and Brook 1984], where $a$ equals 5 [Rahaman and De Jonghe 1987], and $\mathrm{P}$ is the porosity, the values of $\phi$ and $\phi_{c}$ are determined.

The ratio of the center-to-center pore spacings, $\left(x / x_{c}\right)^{n}$, of the as-received over the pre-coarsened $\mathrm{ZnO}$ is plotted versus temperature in Fig. 5.16. The figure is divided into a lower region where $\left(x / x_{c}\right)<1$, and an upper region where $\left(x / x_{c}\right)>1$. As seen in the figure, for most of the temperature range the center-to-center pore spacing of the pre-coarsened $\mathrm{ZnO}$ is larger than that of the as-received $\mathrm{ZnO}$. However, as the temperature increases, the ratio of $\left(x / x_{c}\right)$ increases steadily. At approximately $890^{\circ} \mathrm{C}$, where the relative densities of the as-received and pre-coarsened samples are 
approximately 0.9 , the center-to-center pore spacing ratio crosses into the upper region where the spacing of the pre-coarsened sample now remains smaller than that of the as-received sample.

The pore spacing of the as-received sample surpasses the pore spacing of the pre-coarsened sample only in the later stages of sintering. This behavior is also present in $\mathrm{MgO}$ samples, where at 0.8 density the grain size of the conventionally sintered sample is smaller than that of the pre-coarsened sample but at 0.93 density, the grain size of the two-step sintered sample is actually smaller than that of the single-step sintered sample.

\subsubsection{Modeling of the Evolution of the Interpore Spacing, $x(t, T)$}

In the previous chapter on coarsening at constant heating rate, an expression for the evolution of the interpore spacing, $x(t, T)$ was developed. The form of the expression was taken to be analogous to that describing grain growth in porous systems, although it must be stressed that the pore spacing, $x(t, T)$ and the grain size do not necessarily develop proportionately. The expression for the pore spacing evolution, $x(t, T)$, the

parameter of importance in Eqn. 5.4, recognizes two contributions to pore spacing increase: those arising from non-densifying processes and those arising from densifying processes. At constant heating rate with

$$
T=T_{0}+\alpha t \quad \text { Eqn. } 5.8
$$

and

$$
d T=\alpha d t \quad \text { Eqn. } 5.9
$$

the pore spacing relationship during densification takes the following form (Eqn. 4.10): 
$x^{m}(T)=x_{0}^{m}+\frac{A_{0}}{\alpha} \int_{T_{0}}^{T} \exp \left(-\frac{Q_{l}}{k T}\right) d T+\frac{B_{0}}{\alpha} \int_{T_{0}}^{T} \exp \left(-\frac{Q_{h}}{k T}\right) d T$

Eqn. 5.10

where $x_{0}$, is the starting center-to-center pore spacing at $T_{0}, m$ is the coarsening exponent ( $\mathrm{m}=3$ for $\mathrm{ZnO}$ [Rahaman and $\mathrm{De}$ Jonghe 1987b]), $\alpha$ is the heating rate, $A_{0}$ is the low temperature prefactor associated with low activation energy processes, $Q_{1}$ is the low activation energy, $B_{0}$ is the high temperature prefactor associated with high activation energy processes, and $\mathrm{Q}_{h}$ is the high activation energy.

While the detailed relationship of this expression to the actual micro-processes has yet to be established, it could describe the evolution of $x(t, T)$ well and can at least lend itself to some qualitative predictions. For example, if evaporation-condensation or surface diffusional transport is a coarsening mechanism, it can only be active as long as a continuous pore network exists and will gain in importance with increasing pore channel size [Herring 1950]. This network would increase the magnitude of $A_{0}$ with respect to $B_{0}$, where $Q_{l}$ corresponds to that coarsening mechanism.

For the numerical calculation of the relative evolution of the pore spacing in the pre-coarsened compared to the as-received $\mathrm{ZnO}$, the same parameters were used as determined earlier in section 4.4.3. The ratio $\left(x / x_{c}\right)^{m}$ can then be determined with $x_{C O}=1.7 x_{0}$ appropriate to the present data.

\footnotetext{
- $A_{0}=5.6 \times 10^{-9}$

$B_{0}=1.0 \times 10^{0}$

$\mathrm{Q}_{1}=15 \mathrm{kcal} / \mathrm{mole}$

$\mathrm{Q}_{\mathrm{h}}=50 \mathrm{kcal} / \mathrm{mole}$
} 


$$
\left(\frac{x}{x_{c}}\right)^{m}=\frac{x_{o}^{m}+\frac{A_{0}}{\alpha} \int_{T_{0}}^{T} \exp \left(-\frac{Q_{1}}{k T}\right) d T+\frac{B_{0}}{\alpha} \int_{T_{0}}^{T} \exp \left(-\frac{Q_{h}}{k T}\right) d T}{x_{c o}^{m}+\frac{A_{c o}}{\alpha} \int_{T_{0}}^{T} \exp \left(-\frac{Q_{1}}{k T}\right) d T+\frac{B_{c o}}{\alpha} \int_{T_{0}}^{T} \exp \left(-\frac{Q_{h}}{k T}\right) d T} \text { Eqn. 5.11 }
$$

The choice of $A_{c o}$ and $B_{c o}$ in Eqn. 5.11 must be such that the evolution of the calculated ratio reflects, at least qualitatively, the experimental one. Since significant coarsening has been found to occur at relatively low temperatures for this $\mathrm{ZnO}$ powder $(\mathrm{CHR})$, the trend of the experimental ratio $\left(x / x_{c}\right)^{n^{*}}$ up to about $890^{\circ} \mathrm{C}$ (corresponding to a sintered density of about 0.9 ), can only be reproduced if $A_{c o}>$ $A_{0}$. The subsequent increase of the experimental $\left(x / x_{c}\right)^{n}$ to values higher than 1 can only result if $\mathrm{B}_{\mathrm{co}}<\mathrm{B}_{\mathrm{O}}$. Different choices for these parameters and calculated trends are illustrated in Fig. 5.17.

The effects of pre-coarsening on the evolution of the pore spacing can therefore be summarized as facilitating the low temperature coarsening but suppressing the high temperature coarsening.

\subsubsection{Application of Pre-coarsening and Two-step Sintering}

The results of the present study can be put in the context of the qualitative evolution of porosity and grain size distributions. It should be expected that any system will tend towards the steady state distribution described by the theories of Slyozov \& Lifshitz [1961], and Wagner [1961] (LSW). The initial distribution of pore sizes and grain sizes and the relative importance of coarsening and densification of the compact

$\mathrm{n}$, the densification exponent, is determined by the sintering mechanism and $\mathrm{m}$, is the observed grain growth exponent. In general the two exponents are taken to be equivalent [Vieira and Brook 1984] but it is noted that $\mathrm{n}$ does not necessarily have to equal $\mathrm{m}$. 
will determine at which sintered density and mean grain size or mean pore spacing this steady state distribution is reached. Technical powders and compacts, such as the ones examined here, will most often have a structural distribution that is significantly broader than the LSW distribution; for monosized powder systems, the opposite is the case. There is therefore no merit in pre-coarsening those systems that have a structural distribution equal to or narrower than the LSW one. However, for technical powder compacts, the present results demonstrate that pre-coarsening will improve the structural distribution. Immediate derisification of the compact before homogenization is likely to result in the production of sintering damage driven by differential densification, leading to the view that such immediate densification delays reaching the LSW distribution. At the same time, heterogeneity promotes the generation of mixtures of open and closed porosity regions at sintered densities lower than those of more homogeneous systems, as would follow from the work of Carpay [1977]. A qualitative limit on pre-coarsening must, however, be immediately recognized: if the initial distribution is too far removed from the LSW distribution then pre-coarsening needed to reach the LSW distribution would be extensive and the mean value of $x$ wou!d have to be so large as to inhibit further densification. Quantitative limits on distributions are more difficult to determine, especially since clear definitions or quantitative measurements of relevant "heterogeneity" have yet to be formulated.

In practice, the usefulness of the proposed two-step sintering process is readily evaluated by a few trial experiments. The results of the experiments presented here also indicate that, within limits, technical powders can be used effectively by including a simple isothermal pre-coarsening treatment to produce improved microstructures in sintered bodies; resorting to the use of monosized powders or convoluted heat schedules is unnecessary. 


\subsection{Summary}

$\mathrm{MgO}$ and $\mathrm{Al}_{2} \mathrm{O}_{3}$ were sintered by two types of processes: a conventional isothermal sintering, and a two-step sintering with an initial low temperature pre-coarsening treatment before conventional isothermal sintering. The final microstructure from two-step sintering is more uniform and finer than that of compacts sintered conventionally.

$\mathrm{ZnO}$ compacts were pre-coarsened at $450^{\circ} \mathrm{C}$ for 90 hours, the resulting grain size was 1.7 times larger than the starting grain size, but the standard deviation of the grain size distribution was narrower than that of the as-received powder. The pre-coarsening was completed with virtually no increase in the overall density for the $\mathrm{ZnO}$ compacts. $\Sigma_{c}$, the sintering stress of the pre-coarsened $\mathrm{ZnO}$, is approximately 0.8 of the sintering stress of the as-received $\mathrm{ZnO}, \Sigma$, decreasing less than expected from the grain size increase.

As a function of temperature the densification and creep strain rates of the pre-coarsened sample started out lower than that of the as-received sample but increased steadily as sintering proceeded, until the strain rate of the pre-coarsened sample exceeded that of the as-received sample.

A computational model has been used with two components of coarsening to describe the pore spacing evolution, one with a low activation energy and the other with a high activation energy. Pre-coarsening increases the low iemperature prefactor but decreases the high temperature prefactor. This behavior has been attributed to an increase in uniformity which results from pre-coarsening. The increased uniformity allows the system to stay in the open porosity state longer, delaying or inhibiting additional coarsening (grain growth) during the final stage of densification. 
Two-step sintering is especially beneficial for non-uniform powders with size distributions that are wider than the LSW one. The process, incorporating an initial low temperature pre-coarsening treatment prior to densification, offers a simple and convenient method of making more uniform ceramic bodies without resorting to specialized powders or complicated heat schedules. 


\section{REFERENCES}

1. E.H. Aigeltinger, "Relating Microstructure and Sintering Force," Inter. J. Powder Met. and Powder Tech., 11 [3] 195-203 (1975).

2. B.H. Alexander and R.W. Balluffi, "The Mechanism of Sintering of Copper," Acta metall, 5 666-77 (1957).

3. M.F. Ashby, "A First Report on Sintering Diagrams," Acta metall, 22 274-89 (1974).

4. H.V.Atkinson, "Theories of Normal Grain Growth in Pure Single Phase Systems," Acta metall, 36 [3] 469-91 (1988).

5. L.K. Barrett and C.S. Yust, "Progressive Shape Changes of the Void During Sintering", Trans. Metall. Soc. AIME. 239 1172-80 (1967).

6. E.A. Barringer, R.J. Brook, and H.K. Bowen, "The Sintering of Monodisperse $\mathrm{TiO}_{2}$," in Materials Science Research Vol. 16, ed. G.C. Kuczynski, A.E. Miller, G.A. Sargent (Plenum Press, New York 1984) p. 1-21.

7. (a) W. Beere, "A Unifying Theory of the Stability of Penetrating Liquid Phases and Sintering Pores," Acta metall, 23 [1] 131-39 (1975).

8. (b) W. Beere, "The Second Stage Sintering Kinetics of Powder Compacts," Acta metall, 23 [1] 139-45 (1975).

9. (c) W. Beere, "Diffusional Flow and Hot Pressing: A Study on MgO," J. Mat. Sci, 10 1434-40 (1975).

10 R.K. Bordia and G.W. Scherer, "On Constrained Sintering - III. Rigid Inclusions," Acta metall, 36 [9] 2411-16 (1988).

11. P. Bross and H.E. Exner, "Computer Simulation of Sintering Processes," Acta metall, 27 [6] 1013-20 (1979). 
12. R.J. Brook, "Controlled Grain Growth," in Treatise on Materials Science and Technology Vol 9, ed. F.F.Y. Wang (Academic Press, New York 1976) p. 331-65.

13. (a) R.J. Brook, "Hot Pressing Dilatometry in the Study of Sintering Mechanisms," in Sintering - Theory and Practice, Material Science Monographs, Vol 14, ed. D. Kolar, S. Pejovnik, and M.M. Ristic (Elsevier Scientific Publishing Company, Amsterdam, 1982) p. 585-90.

14. (b) R.J. Brook, "Fabrication Principles for the Production of Ceramics with Superior Mechanical Properties," Proc. Brit. Ceram. Soc., 32 (1982) p. 7-24.

15. J.E. Burke "The Science and Technology of Sintering," in The Physics and Chemistry of Ceramics, ed. C. Klingsberg (Gordon and Breach, New York 1962) p. 165-78.

16. J.E. Burke and J.H. Rosolowski, "Sintering", in Treatise on Solid State Chemistry, Vol 4., ed. N.B. Hannay (Plenum Press, New York 1976) p. 621-59.

17. R.M. Cannon, "On the Effects of Dihedral Angles and Pressure on the Driving Force for Pore Growth or Shrinkage", unpublished manuscript, June, 1981.

18. R.M. Cannon and W.C. Carter, "Interplay of Sintering Microstructures, Driving Forces, and Mass Transport Mechanisms," J. Am. Ceram. Soc., 72 [8] 1550-55 (1989).

19. E. Carlstrom, A.-K. Tjernlund, L. Hermansson, auld R. Carlsson, "Influence of Powder and Green Compact Characieristics on Microstructure of Sintered Alpha-SiC," Proc. Brit. Ceram. Soc., 33 89-100 (1983).

20. F.M.A. Carpay, "The Effect of Pore Drag on Ceramic Microstructure," in Ceramic Microstructure 76, ed. R.M. Fulrath and J.A. Pask (Westview Press, Colorado 1977) p. 261-75. 
21. M.-Y. Chu, L. C. De Jonghe, and M. N. Rahaman, "Effect of Temperature on the Densification/Creep Viscosity During Sintering," Acta metal:. 37 [5] 1415-20 (1989).

22. R.L. Coble, "Initial Sintering of Alumina and Hematite," J. Am. Ceram. Soc., 41 55-60 (1958).

23. (a) R.L Coble, "Sintering Crystalline Solids. I. Intermediate and Final State Diffusion Models," J. Appl. Phys. 32 [5] $787-92$ (1961).

24. (b) R.L. Coble, "Sintering Crystalline Solids. II. Experimental Test of Diffusion Models in Powder Compacts," J. Appl. Phys. 32 [5] 793-99 (1961).

25. R.L. Coble, "A Model for Boundary Diffusion Controlled Creed in Polycrystalline Materials," J. Appl Phys. 34 [6] 1679-82 (1963).

26. R.L. Coble, "Diffusion Models for Hot Pressing with Surface Energy and Pressure Effects as Driving Forces," J. Appl. Phys. 41 [12] 4798-807 (1970).

27. R.L. Coble and R.M. Cannon, "Current Paradigms in Powder Processing," in Materials Sciences Research Vol.11, ed. H. Palmour III, R.F. Davis, T.M. Hare (Plemum Press, New York 1978) p. 151-70.

28. S.C. Coleman and W.B. Beere, "The Sintering of Open and Closed Porosity in $\mathrm{UO}_{2}$," Phil. Mag. 31 [4] 1403-13 (1975).

29. I. B. Cutler, "Sint ririb of Glass Powders During Constant Rates of Heating," $J$. Am. Ceram. Soc., 52 [1] 14-7 (1969).

30. R.T. DeHoff, R.A. Rummel, H.P. I aBuff, and F.N. Riines, "The Relationship between Surface Area and Density in the Second-Stage Sintering of Metals", Modern Developments In Powder Metallurgy !'ol.1 (Plenum Press, New York 196 J) p. 310-31. 
31. R.T. DeHoff, "A Cell Model for Microstructural Evolution during Sintering," in Materials Science Research Vol 16, ed. G.C. Kuczynski, A.E. Miller and G.A. Sargent (Plenum Press, New York, 1984) p. 23-34.

32. (a) L.C. De Jonghe and M.N. Rahaman, "Loading Dilatometer," Rev. Sci. Instrum. 55 [12] 2007-10 (1984).

33. (b) L.C. De Jonghe and M.N. Rahaman, "Pore Shrinkage and Sintering Stress," Bull. Am. Ceram. Soc., 67 [10] c214-15 (1984).

34. L.C. De Jonghe and M.N. Rahaman, "Sintering Stress of Homogeneous and Heterogeneous Powder Compacts," Acta metall, 36 [1] 223-29 (1988).

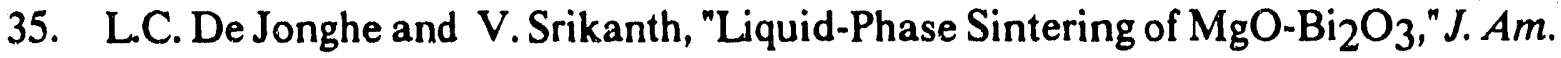
Ceram. Soc. 71 [7] c356-58 (1988).

36. L.C. De Jonghe, M.-Y. Chu, and M.K.F. Lin, "Pore Size Distribution, Grain Growth, and the Sintering Stress", J. Mat. Sci. 24 4403-08 (1989).

37. L.C. De Jonghe, M.N. Rahaman, and C.H. Hsueh, "Transient Stresses in Bimodal Compacts During Sintering," Acta Metall, 34 [7] 1467-71 (1986).

38. R.L. Eadie, G.C. Weatherly and K.T. Aust, "A Study of Spherical Silver Powder - I. The Intermediate Stage," Acta metall, 26759 67 (1978).

39. R.L. Eadie, D.S. Wilkinson, and G.C. Weatherley, "The Rate of Shrinkage during the Initial Stage of Sintering," Acta metall, 22 1185-95 (1974).

40. A.G. Evans, "Considerations of Inhomogeneity Effects in Sintering," J. Am. Ceram. Soc., 65 [10] 497-501 (1982).

41. A.G. Evans and C.H. Hsueh, "Behavior of Large Pores During Sintering and Hot Isostatic Pressing," J. Am. Ceram. Soc., 69 [6] 444-48 (1986). 
42. H.E. Exner and P. Bross, "Material Transport Rate and Stress Distribution during Grain Boundary Diffusion Driven by Surface Tension," Acta metall, 27 [6] 1007-12 (1979).

43. H.E. Exner and G. Petzow, "Shrinkage and Rearrangement During Sintering of Glass Spheres," in Materials Science Research Vol 16, ed. G.C. Kuczynski, A.E. Miller and G.A. Sargent (Plenum Press, New York, 1984) p. $279-93$.

44. H.E. Exner and G. Petzow, "A Critical Evaluation of Shrinkage Equations," in Materials Science Research Vol. 13, ed. G.C. Kuczynski (Plenum Press, New York 1980) p. 107-20.

45. H.E. Exner, G. Petzow, and P. Wellner, "Problems in the Extension of Sintering Theories to Real Systems," in Sintering and Related Phenomena 13, ed. G.C. Kuczynski (Plenum Press, New York 1976) p. 351-62.

46. J. Frenkel, "Viscous Flow of Crystalline Bodies Under the Action of Surface Tension," J. Phys. (USSR), 9 [5] 385-91 (1945).

47. R.M. German, Powder Metallurgy Science (Metal Powder Industries Federation, Princeton 1984) Chap. 6.

48. R.A. Gregg and F.N. Rhines, "Surface Tension and the Sintering Force in Copper," Metall. Trans. 4[4] 1365-74 (1973).

49. C. Greskovich and K.W. Lay, "Grain Growth in Porous Compact," J. Am. Ceram. Soc., 55 [3] 142-46 (1972).

50. T. K. Gupta and R. L. Coble, "Sintering of $\mathrm{ZnO}$ : I, Densification and Grain Growth," J. Am. Ceram. Soc., 51 [9; 521-25 (1968).

51. C.A. Handwerker, R.M. Cannon, and R.L. Coble, "Final-Stage Sintering of MgO," in Advances in Ceramics Vol.10, ed. W.D. Kingery (American Ceramics Soc., Ohio 1984) p. 619-43. 
52. (a) C. Herring, "Effect of Change of Scale on Sintering Phenomena," J. Appl. Phys., 21 301-03 (1950).

53. (b) C. Herring, "Diffusional Viscosity of a Polycrystalline Solid," J. Appl. Phys., 21 437-45 (1950).

54. M. Hillert, "On the Theory of Normal and Abnormal Grain Growth," Acta Metall, 13 227-38 (1965).

55. C.H. Hsueh and L.C. De Jonghe, "Particle Rotation in Early Sintering," Bull Am. Ceram. Soc., 67 [10] c215-17 (1984).

56. (a) C.H. Hsueh, A.G. Evans, R.M. Cannon, and R.J. Brook, "Viscoelastic Stresses and Sintering Damage in Heterogeneous Powder Compacts," Acia Metall, 34 [5] 927-36 (1986).

57. (b) C.H. Hsueh, A.G. Evans, and R.M. McMeeking, "Influence of Multiple Heterogeneities on Sintering Rates," J. Am. Ceram. Soc., 69 [4] c64-66 (1986).

58. K.S. Hwang and R.M. German, "Analysis of Initial Stage Sintering by Compuier Simulation," in Materials Science Research Vol. 16, ed. G.C. Kuczynski, A.E. Miller, and G.A. Sargent (Plenum Press, New York 1984) p. 35-47.

59. D.L. Johnson, "New Method of Obtaining Volume, Grain-Boundary, and Surface Diffusion Coefficients from Sintering Data," J. Appl Phys., 40 [1] 192-200 (1969).

60. D.L. Johnson, "A General Model for the Intermediate Stage of Sintering, "J. Am. Ceram. Soc. 53 [10] $574-77$ (1970).

61. D.L. Johnson, "Recent Developments in the Theoretical Analysis Solid State Sintering", in Materials Science Monographs, Vol 14, ed. D. Kular, S. Pejovnik, and M.M. Ristic (Elsevier Scientific Publishing Company, Amsterdam, 1982) p. 17-26. 
62. D.L. Johnson, "Ultra-Rapid Sintering", in Materials Science Research Vol. 16, ed. G.C. Kuczynski, A.E. Miller and G.A. Sargent (Plenum Press, New York 1984) p. 243-52.

63 D.L. Johwow "Sintering and Creep of Copper, Nickel, and Alloys," in EPRI Report, (frif, Palo Alto 1988).

64. D.L. Johnson and Cutler, "Diffusion Sintering I, Initial Stage Sintering Models and Their Application to Shrinkage of Powder Compacts," J. Am. Ceram. Soc. 46 [11] $541-50$ (1963).

65. W.A. Kaysser and A. Lenhart, "Optimalization of Densification of $\mathrm{ZnO}$ during Sintering," Powder Metal Inter. 13 [3] 126-28 (1981).

66. B.J. Kellet and F.F. Lange, "Thermodynamics of Densification: I, Sintering of Simple Particle Arrays, Equilibrium Configurations, Pore Stability, and Shrinkage," J. Am. Ceram. Soc., 72 [5] 725-34 (1989).

67. W.D. Kingery and M. Berg, "Study of the Initial Stages of Sintering Solids by Viscous Flow, Evaporation - Condensation, and Self - Diffusion," J. Appl. Phys., 26 [10] 1205-12 (1955).

68. W.D. Kingery and B. Francois, "Grain Growth in Porous Compacts, "J. Am. Ceram. Soc., 48 [10] 546-48 (1965).

69. G.C. Kuczynski, "Self-Diffusion in Sintering of Metallic Particles," Metals Transactions, $169-77$ (Feb. 1945).

70. G.C. Kuczynski, "Statistical Theory of Sintering," Z. Metallkde., 67 [9] 606-10 (1976).

71. G.C. Kuczynski, "Towards the Understanding of the Process of Sintering," in Sintering '85, ed. G.C. Kuczynski, D.P. Uskokovic, H. Palmour III, and M.M. Ristic (Plenum Press, New York, 1987) p. 3-16. 
72. F.F. Lange, "Constrained Network Model for Predicting Densification Behavior of Composite Powders," J. Metr. Resd., 2, [1], 59-65 (1987).

73. F.F. Lange, "Approach To Reliable Powder Processing," Ceram. Trans., 1 1069-83 (1989).

74. F.F. Lange and B. Kellet, "Influence of Particle Arrangement of Sintering Array of Particles," in Science of Ceramic Chemical Processing, ed. L.L. Hench and D.R. Ulrich (Wiley and Sons, 1986) p. 561-74.

75. J. Lee, and D.W. Readey, "Microstructure Development of $\mathrm{Fe}_{2} \mathrm{O}_{3}$ in $\mathrm{HCl}$ Vapor," in Materials Sciences Research Vol. 16, ed. G.C. Kuczynski, A.E. Miller, and G.A. Sargent (Plenum Press, New York 1984) p. 145-57.

76. M. Lin, M.N. Rahaman, and L.C. De Jonghe, "Creep-Sintering and Microstructure Development of Heterogeneous MgO Compacts," J. Am. Ceram. Soc., 70 [5] 360-66 (1987).

77. I.M.Lifshitz and V.V.Slyozov, "The Kinetics of Precipitation from Supersaturated Solid Solutions," Phys. Chem. Solids 19 [1/2] 35-50 (1961).

78. J.K. Mackenzie and R. Shuttleworth, "A Phenomenological Theory of Sintering," Proc. Phys. Soc., 62 833-52 (1949).

79. F.R.N. Nabarro, "Deformation of Crystals by the Motion of Single Ions," in Strength of Solids, (The Physical Society, London, 1948) p. 75-90.

80. F.A. Nichols, "Coalescence of Two Spheres by Surface Dic'. 42 [7] 2805-08 (1966).

81. H. Palmour III and D.R. Johnson, "Phenomenological Model for Rate-Controlled Sintering," in Sintering and Related Phenomena, ed. G.C. Kuczynski, N.A. Hooton, and C.F. Gibbon (Gordon and Breach Science, New York, 1967). p. 779-91. 
82. V. Petrovic, S.M. Radic, D. Uskokovic, and M.M. Ristic, "A Contribution to Investigation of Grain Growth during Oxides Sintering," Sci. Sinter., 17 [1] 81-5 (1985).

83. H. Pickup, U. Eisele, E. Gilbart and R.J. Brook, "Analysis of Coarsening and Densification Kinetics during the Heat Treatment of Nitrogen Ceramics," in Non-Oxide Technical and Engineering Ceramics, ed. S. Hampshire (Elsevier Applied Science, New York 1985) p. 41-51.

84. T. Quadir and D.W. Readey, "Microstructure Evolution in $\mathrm{SnO}_{2}$ and $\mathrm{CdO}$ in Reducing Atmospheres," in Materials Sciences Research Vol. 16, ed. G.C. Kuczynski, A.E. Miller, and G.A. Sargent (Plenum Press, New York, 1984) p. 159-69.

85. M.N. Rahaman and L.C. De Jonghe, "Sintering of CdO Under Low Applied Stress," J. Am Ceram. Soc., 67 [10] c205-07 (1984).

86. M.N. Rahaman and L.C. De Jonghe, and R.J. Brook, "Effect of Shear Stress on Sintering," J. Am. Ceram. Soc., 69 [1] 53-58 (1986).

87. (a) M.N. Rahaman and L.C. De Jonghe, "Effect of Rigid Inclusions on the Sintering of Glass Powder Compacts," J. Am. Ceram. Soc., 70 [12] c348-351 (1987).

88. (b) M.N. Rahaman and L.C. De Jonghe, "Creep-Sintering of Zinc Oxide,"J. Mater. Sci, 22 4326-30 (1987).

89. M.N. Rahaman and L.C. De Jonghe, "Effect of Rigid Inclusions on Sintering," in Fist Internat. Conf. C'eram. Powder Proc. Prod., Orlando (1987).

90. M.N. Rahaman, L.C. De Jonghe, and M.-Y. Chu, "Densification and Shear Deformation in $\mathrm{YBa}_{2} \mathrm{Cu}_{3} \mathrm{O}_{6}+\delta$ Powder Compacts", Advanced Ceramic Materials, 3 [4] 393-97 (1988). 
91. M.N. Rahaman, L.C. De Jonghe, G.W. Scherer, and R.J. Brook, "Creep and Densification During Sintering of Glass Powder Compacts," J. Am. Ceram. Soc., 70 [10] 766-74 (1987).

92. R. Raj, "Analysis of the Sintering Pressure," J. Am. Ceram. Soc. 17 [9] c210-11 (1987).

93. R. Raj and M.F. Ashby, "Intergranular Fracture at Elevated Temperature," Acta metall, 23 653-66 (1975).

94. R. Raj and R.K. Bordia, "Sintering and Behavior of Bimodal Compacts," Acta metall, 32 [7] 1003-19 (1984).

95. D.W. Readey, J. Lee, and T. Quadir "Vapor Transport and Sintering of Ceramics," in Materials Science Research Vil. 16, ed. G.C. Kuczynski, A.E. Miller, and G.A. Sargent (Plenum Press, New York, 1984) p. 115-36.

96. (a) F.N. Rhines and R.T. DeHoff, "Channel Network Decay in Sintering," in Materials Science Research Vol. 16, ed G.C. Kuczynski, A.E. Miller and G.A. Sargent (Plenum Press, New York, 1984) p. 49-61.

97. F.N. Rhines, C.E. Birchenall, and L.A. Hughes, "Behavior o Pores during the Sintering of Copper Compacts," J. of Metals, Trans. AIME, 188 378-88 (1950).

98. W.H. Rhodes, "Agglomerate and Particle Size Effects on Sintering Yttria-Stablized Zirconia," J. Am. Ceram. Soc., 64 [1] 19-22 (1981).

99. G.W.Scherer, "Sintering with Rigid Inclusions, "J. Am. Ceram. Soc., 70[10] 719-25 (1987).

100. G.W. Scherer, "Viscous Sintering of a Bimodal Pore-Size Distribution," J. Am. Ceram. Soc., 67 [11] 709-15 (1984).

101. A.W. Searcy, "Driving Force for Sintering of Particles with Anisotropic Surface Energies," J. Am. Ceram. Soc., 68 [10] c267-68 (1985). 
102. H. Song and R.L. Coble, "The Applicability of Herring's Scaling Law to the Sintering of Powders," in Materials Science Research Vol 16, ed. G.C. Kuczynski, A.E. Miller, and G.A. Sargent (Plenum Press, New York, 1984) p. 63-79.

103. F.B. Swinkels and M.F. Ashby, "A Second Report on Sintering Diagrams," Acta metall, 29 259-81 (1981).

104. K.R. Venkatachari and R. Raj, "Shear Deformation and Densification of Powder Compacts," J. Am. Ceram. Soc., 69 [6] 499-506 (1986).

105. J.M. Vieira, R. J. Brook, "Kinetics of Hot-Pressing: The Semilogarithmic Law," J. Am. Ceram. Soc., 67 [4] 245-49 (1984).

106. C. Wagner, "Theorie der Alterung von Niederschlagen durch Umlosen (Ostwald-Reifung)," Zeitschrift fur Elektrochemie 65 [7/8] 581-91 (1961).

107. M.W. Weiser and L.C. De Jonghe, "Rearrangement During Sintering in Two-Dimensional Array," J. Am. Ceram. Soc., 69 [11] 822-26 (1986).

108. O.J. Whittemore and J.A. Varela, "Pore Distribution and Pore Growth During the Initial Stages of Sintering," in Materials Science Research Vol. 13, ed, G.C. Kuczynski (Plenum Press, New York, 1980) p. 51-60.

109. T.L. Wilson and P.G. Shewmon, "The Role of Interfacial Diffusion in the Sintering of Copper," Met. Trans., 236 48-58 (1966).

110. J.L. Woolfrey and M.J. Bannister, "Nonisothermal Techniques for Studying Initial-Stage Sintering," J. Am. Ceram. Soc., 55 [8] 390-94 (1972).

111. M.F. Yan, R.M. Cannon, Jr., H.K. Bowen, and U. Chowdhry, "Effect o; Grain Size Distribution on Sintered Density," Mat. Sci. and Eng., 60 275-81 (1983).

112. W. S. Young and I. B. Cutler, "Initial Sintering with Constant Rates of Heating," J. Am. Ceram. Soc., 53 [12] 659-63 (1970). 


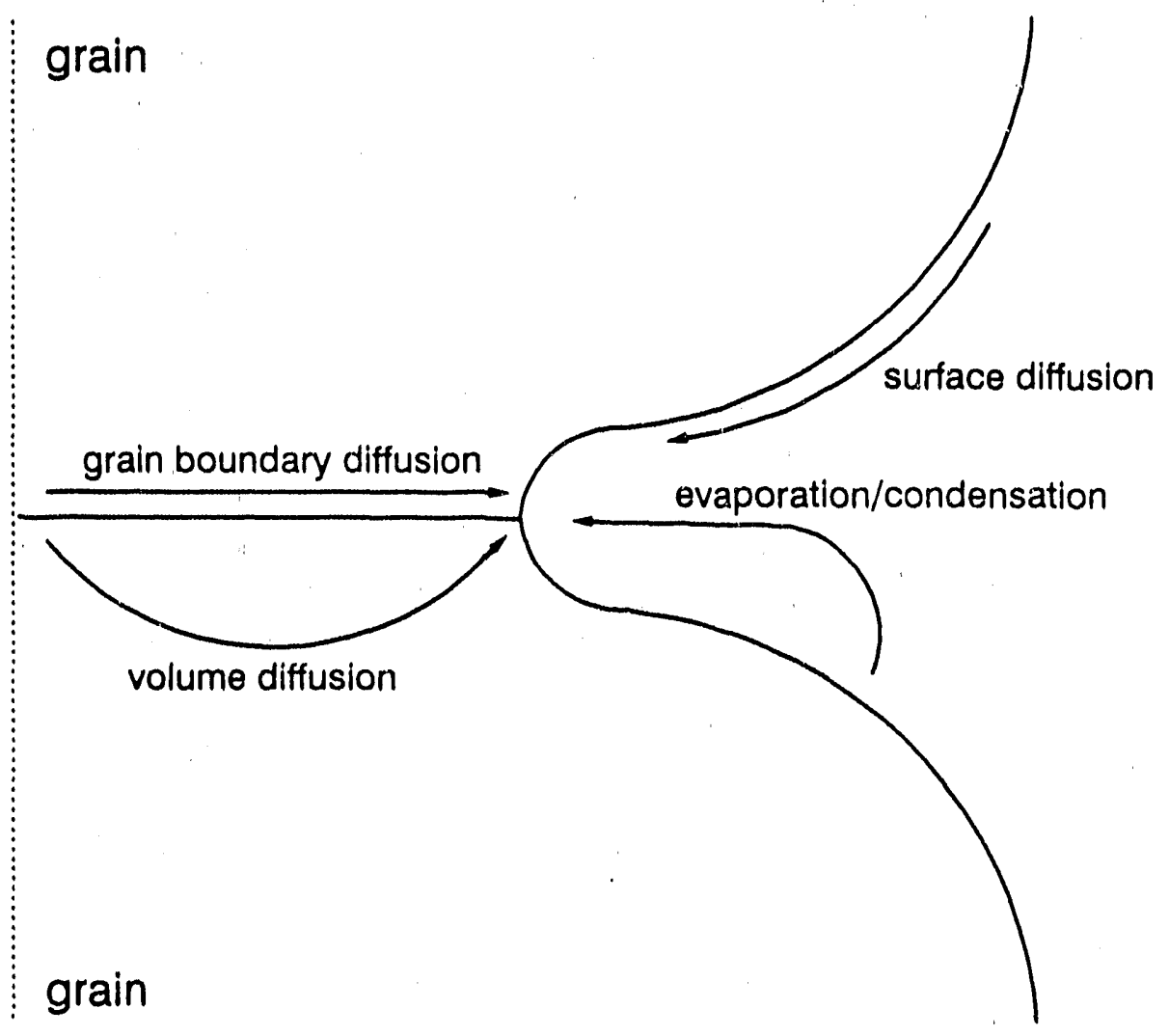

Fig. 2.1 Mechanisms of sintering in a two particle system. 


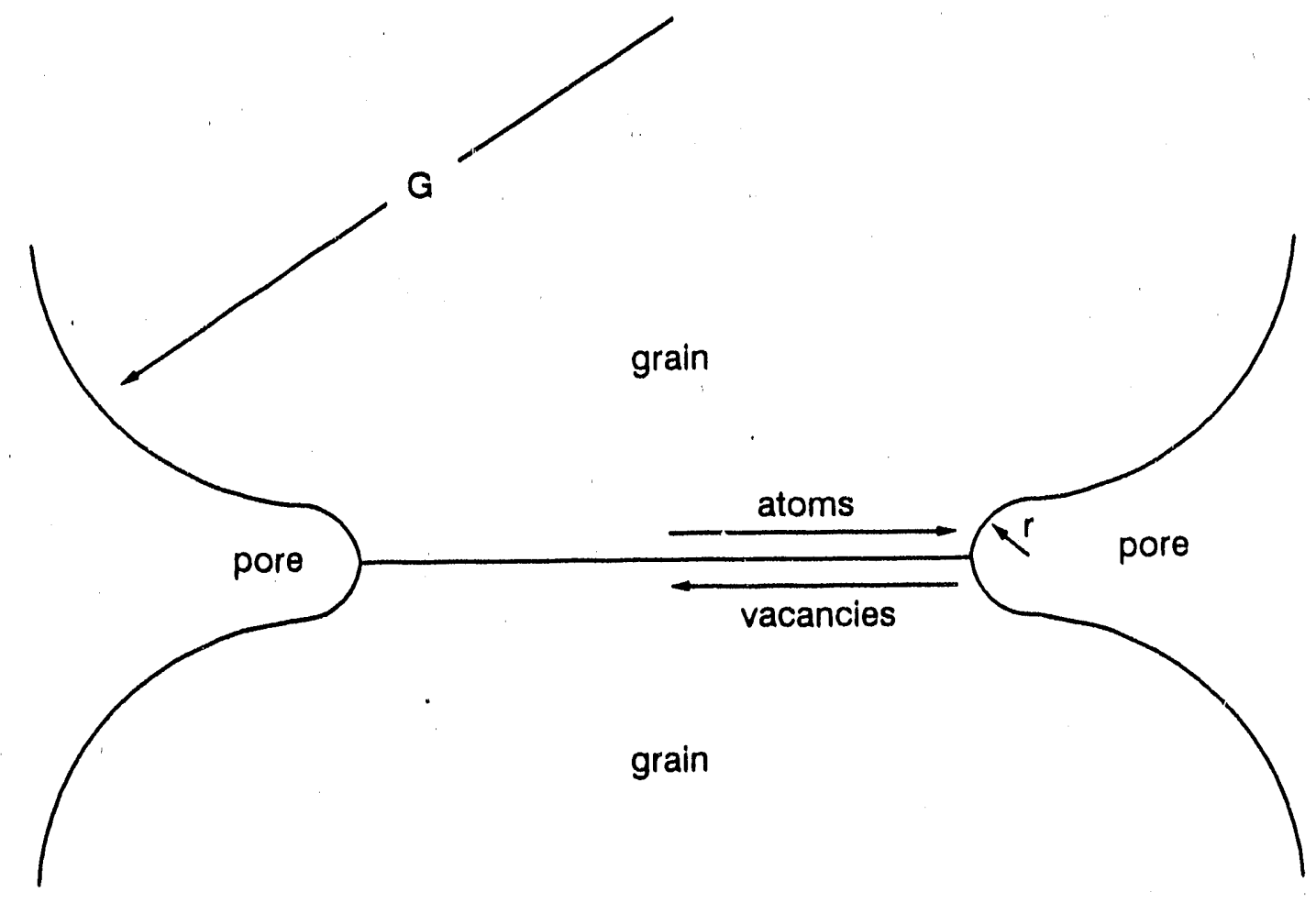

Fig. 2.2 Two particles. 

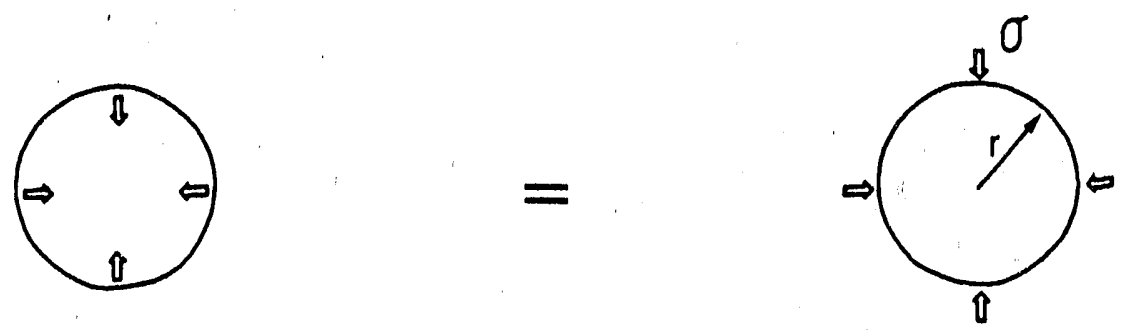

\section{or}

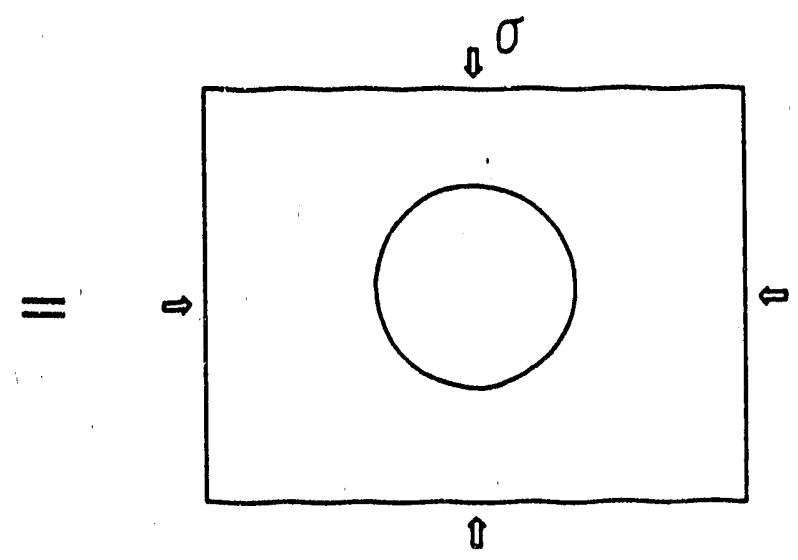

Fig. 2.3 Compressive stress on a spherical pore from the pore curvature and its equivalent stress. 


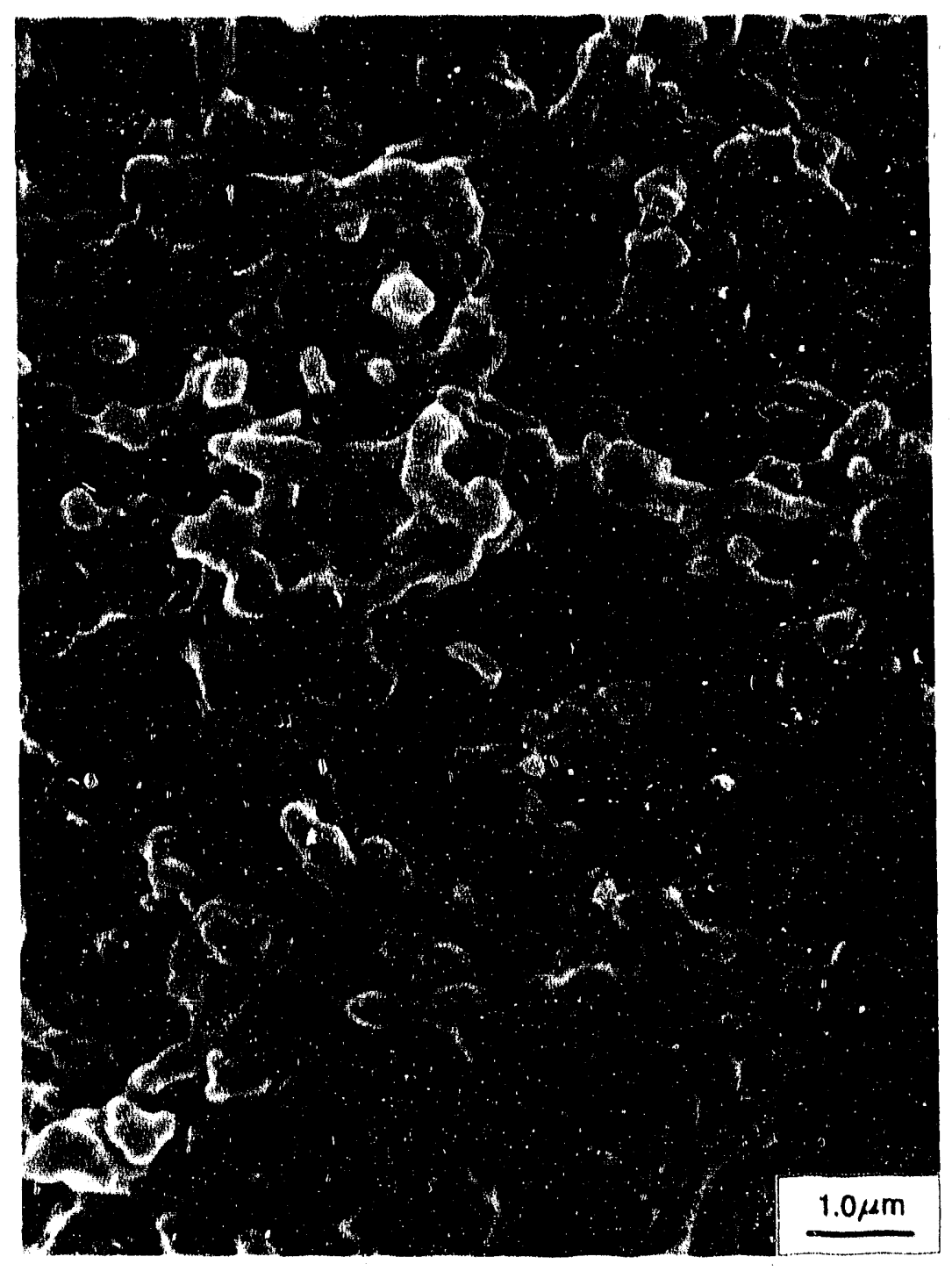

XBB 889-9261a

Fig. 2.4 Epoxy replica of the pore structure of a $\mathrm{ZnO}$ pellet at a relative density of 0.70 . 


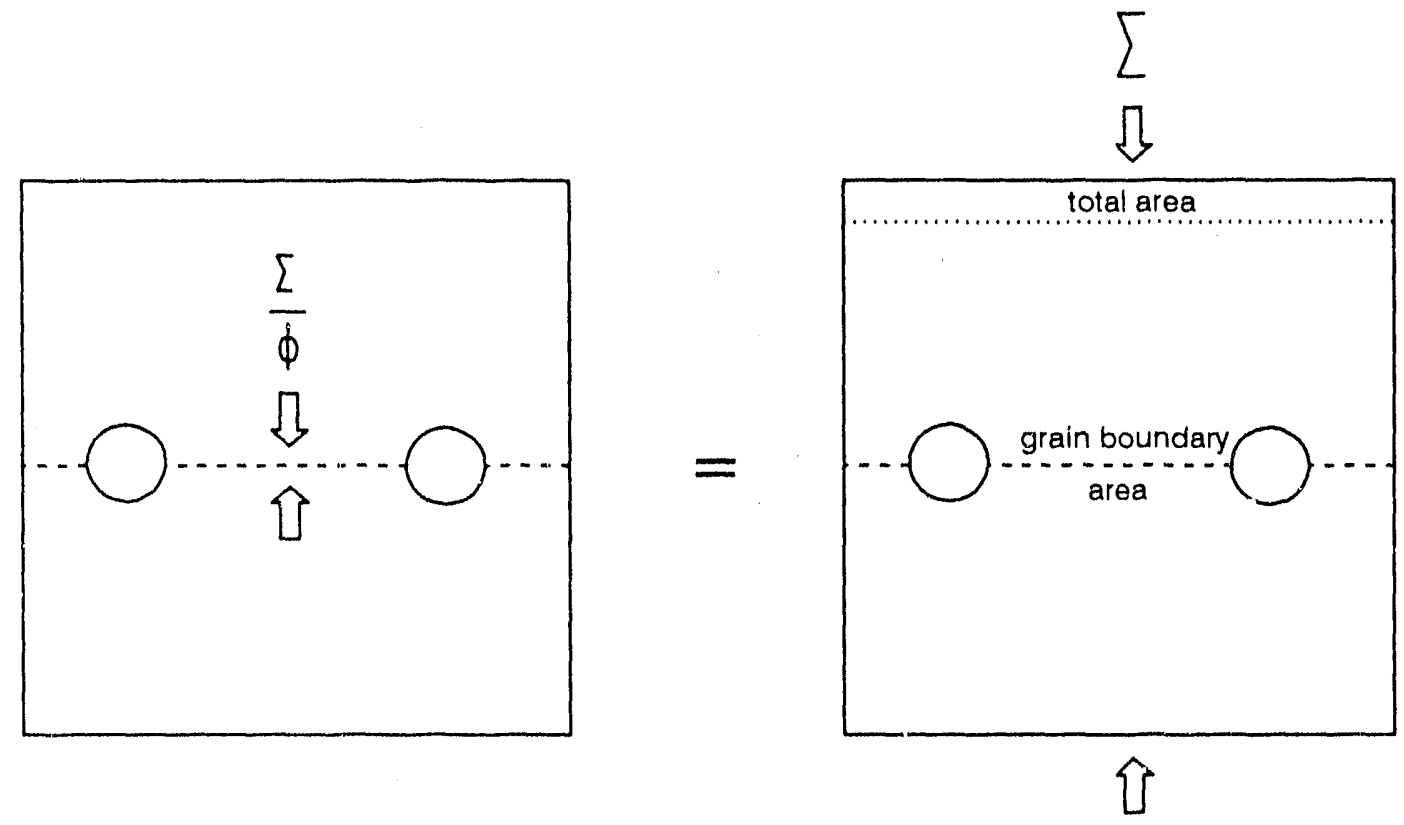

$$
\phi=\frac{\text { total area }}{\text { grain boundary area }}
$$

Fig. 2.5 Grain boundary stress from the system and its equivalent stress. 


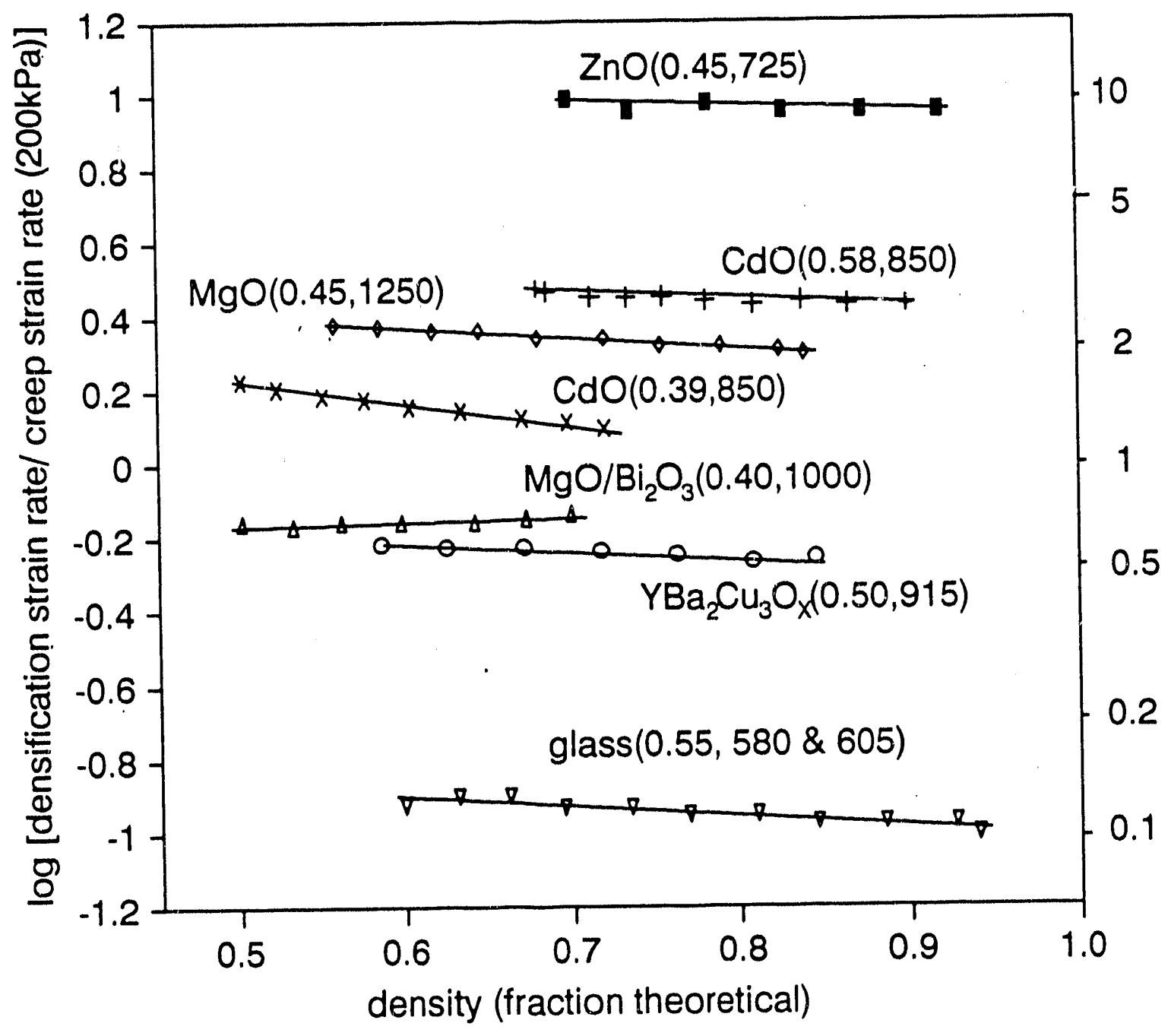

XBL 886-2024

Fig. 3.1 Ratio of the densification strain rate to the creep strain rate versus relative density at a constant applied stress of $0.2 \mathrm{MPa}$. The numbers in parenthesis represent the relative initial density and the sintering temperature $\left({ }^{\circ} \mathrm{C}\right)$. 


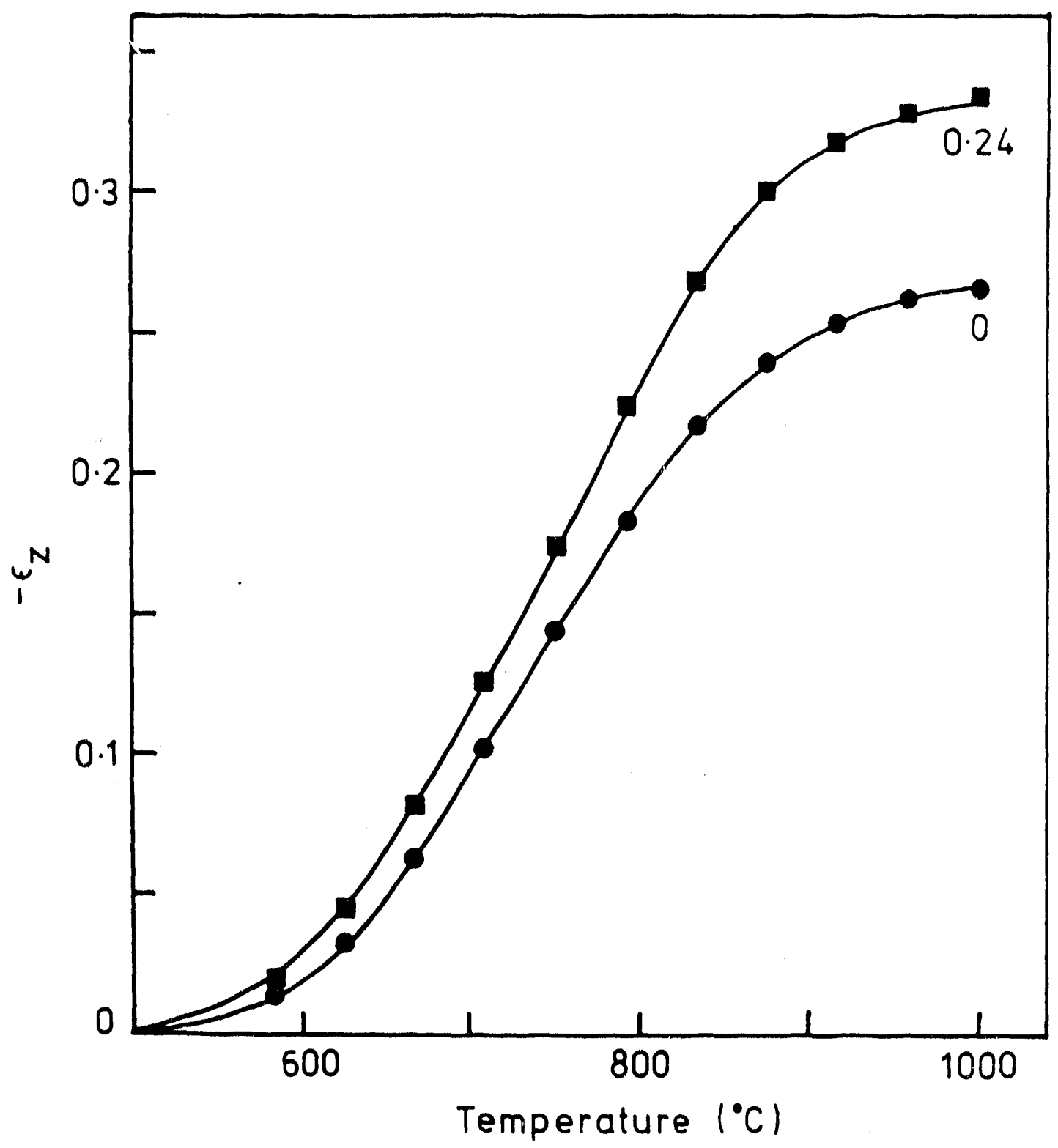

XBL 882-426A

Fig. 3.2 Axial strain versus temperature for $\mathrm{ZnO}$ powder compacts sintered at a constant heating rate of $4^{\circ} \mathrm{C} / \mathrm{min}$ and under the uniaxial stresses shown. 


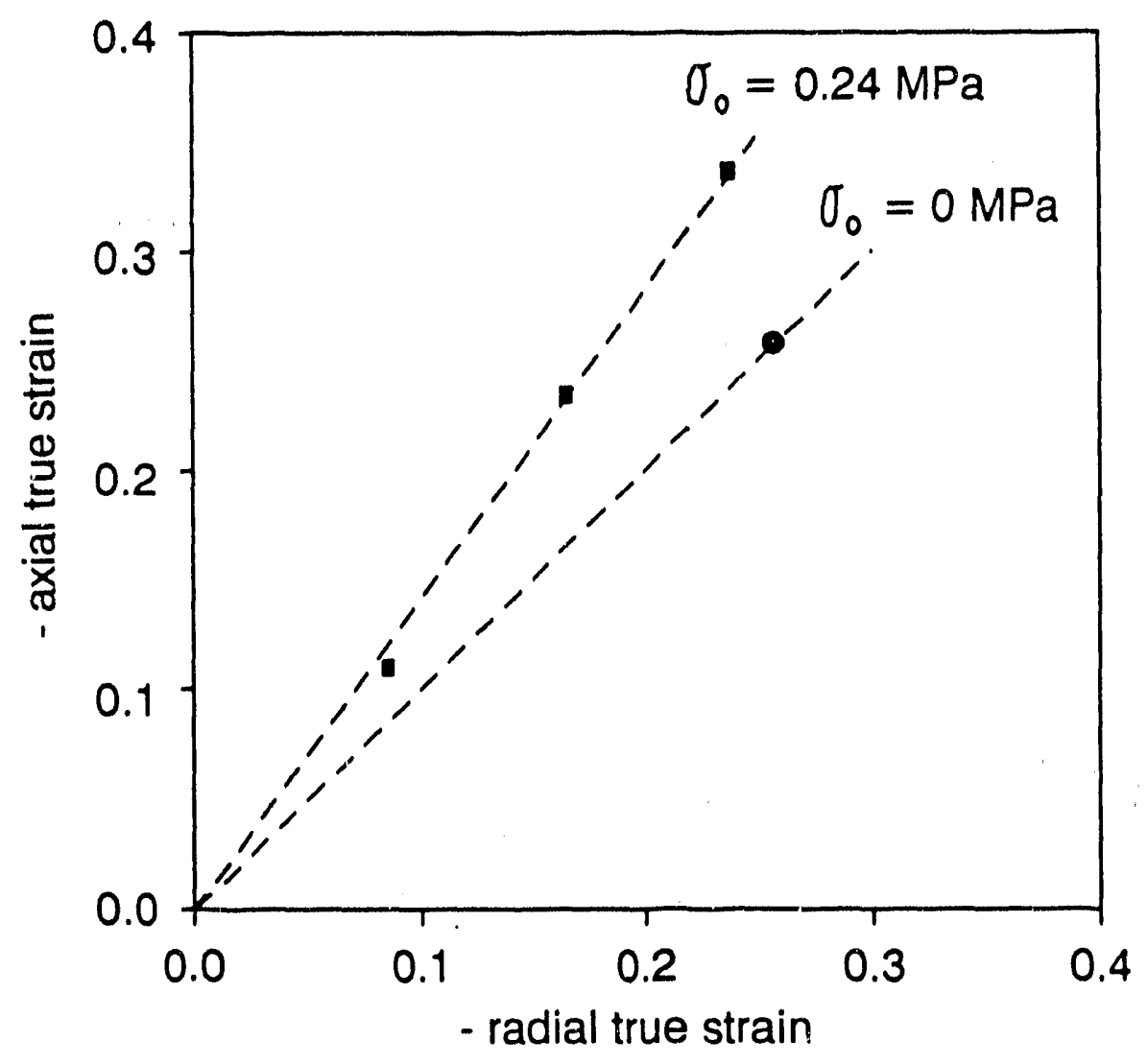

XBL 886-2025

Fig. 3.3 Axial strain versus radial strain for $\mathrm{ZnO}$. 


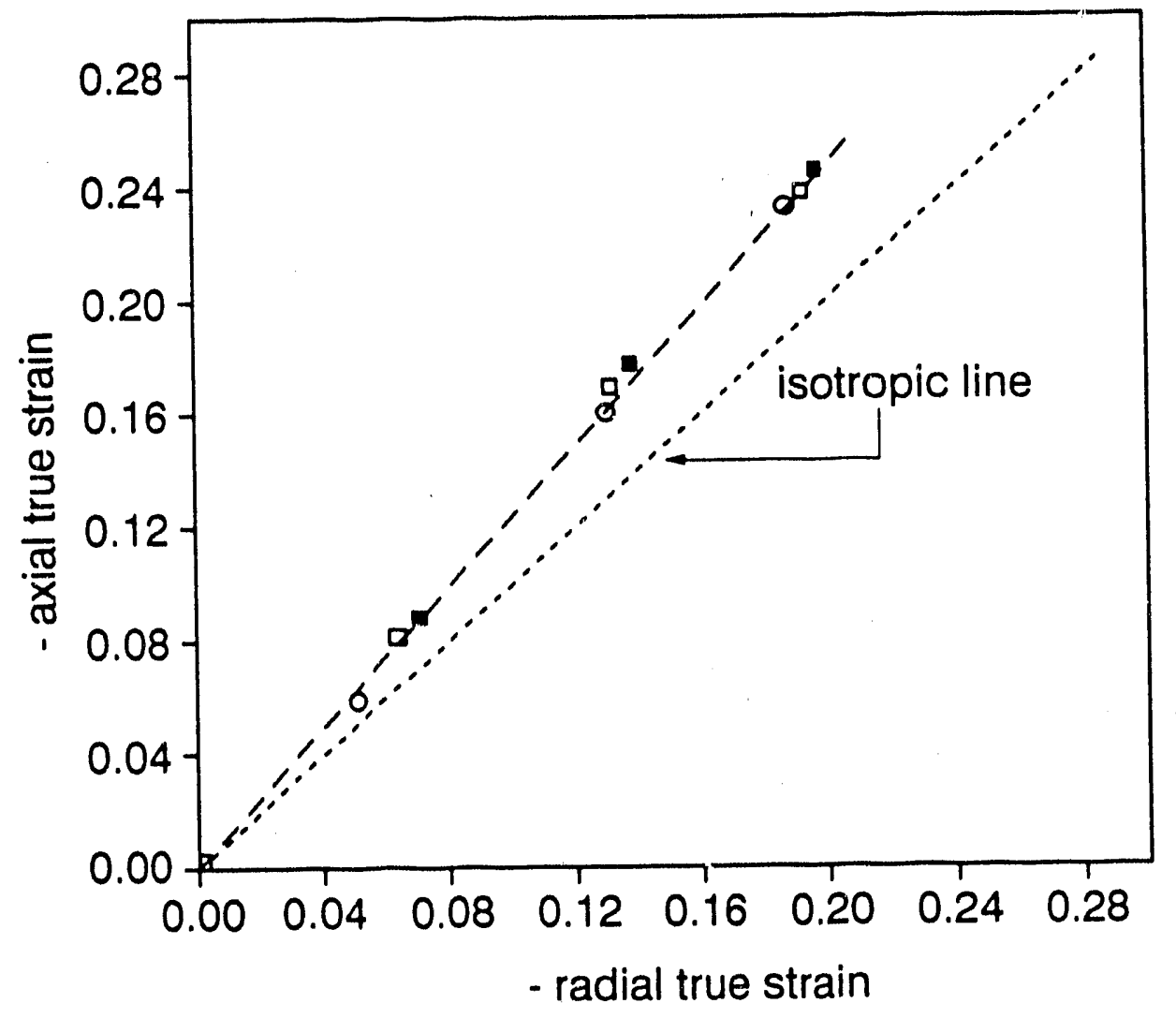

XBL 896-2386

Fig. 3.4 Axial strain versus radial strain under a constant load of $7 \mathrm{~N}$ for $\mathrm{ZnO}: 2^{\circ} \mathrm{C} / \mathrm{min}$ (circle), $4^{\circ} \mathrm{C} / \mathrm{min}$ (square), and $8^{\circ} \mathrm{C} / \mathrm{min}$ (filled square). 


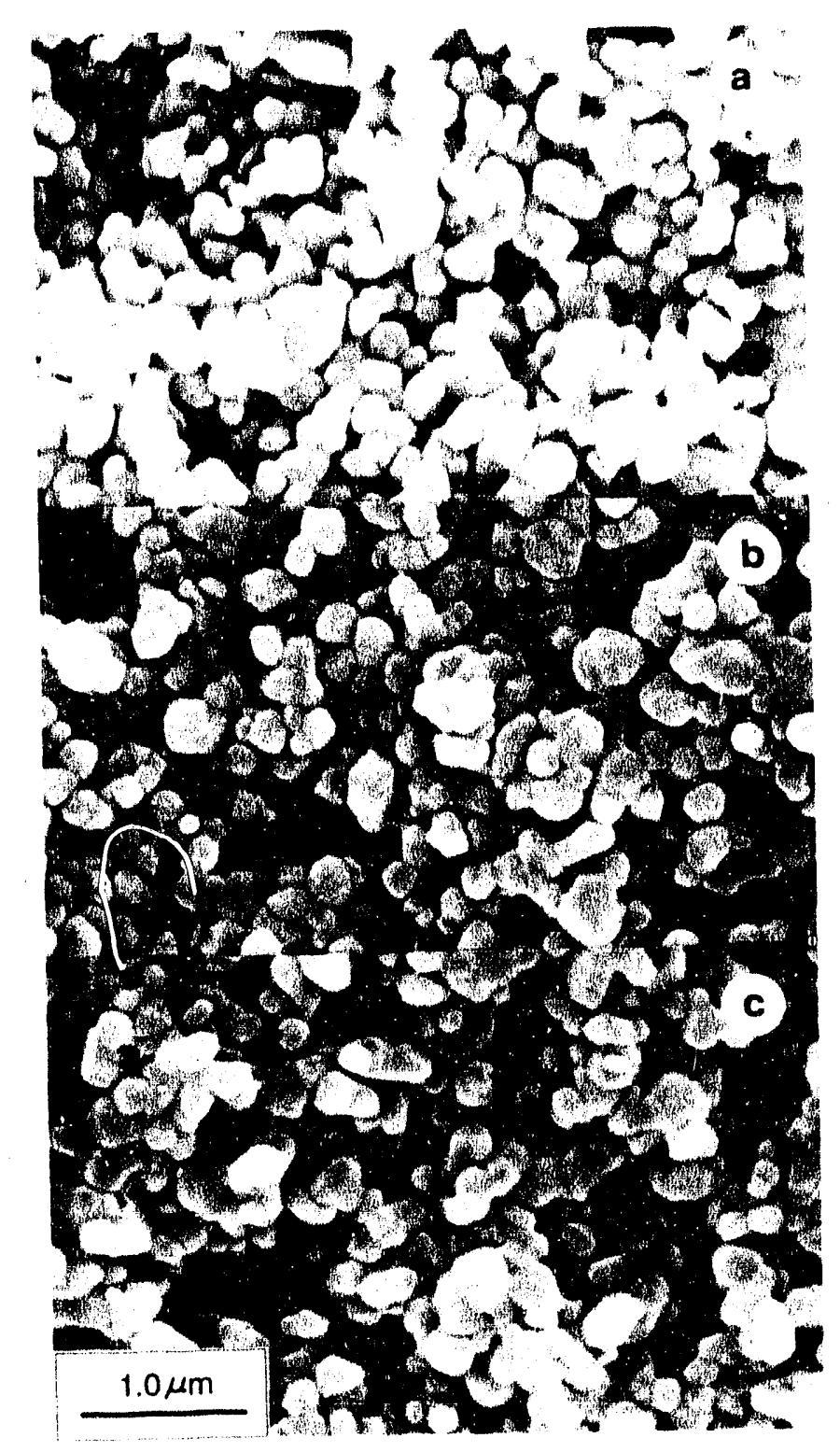

XBB 908.6512

Fig. 3.5 Fracture surface of $\mathrm{ZnO}$ at a relative density of $0.70:$ (a) $2{ }^{\circ} \mathrm{C} / \mathrm{min}$, (b) $4^{\circ} \mathrm{C} / \mathrm{min}$, and (c) $8^{\circ} \mathrm{C} / \mathrm{min}$. 


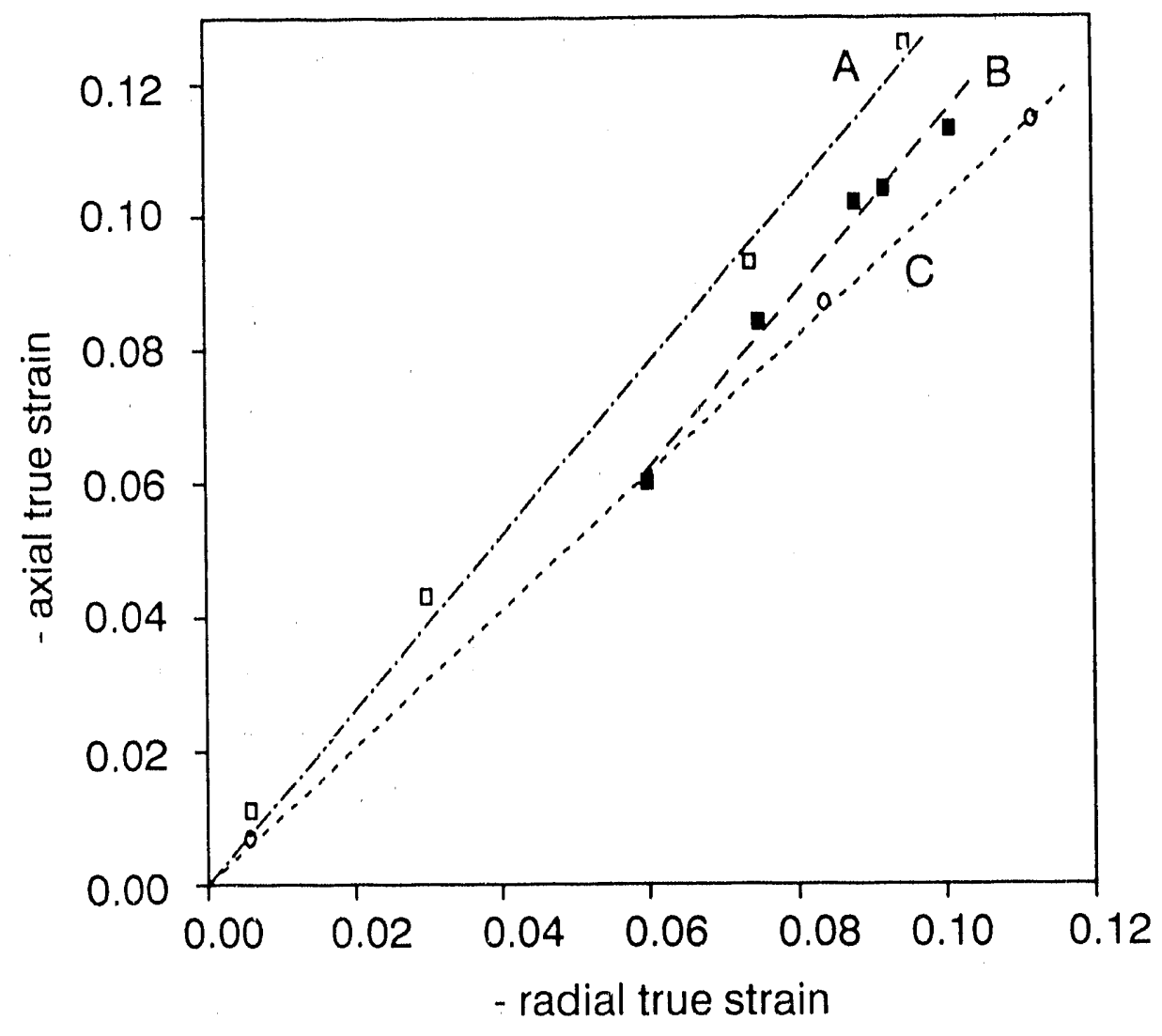

XBL 886-2026

Fig. 3.6 Axial strain versus radial strain for $\mathrm{Al}_{2} \mathrm{O}_{3}$. (A) Heating rate, $4^{\circ} \mathrm{C} / \mathrm{min}$; applied load, $7 \mathrm{~N}$, (B) Isothermal, $1350^{\circ} \mathrm{C}$; applied load, $7 \mathrm{~N}$, and (C) Heating rate, $4^{\circ} \mathrm{C} / \mathrm{min}$; applied load, $0 \mathrm{~N}$. 


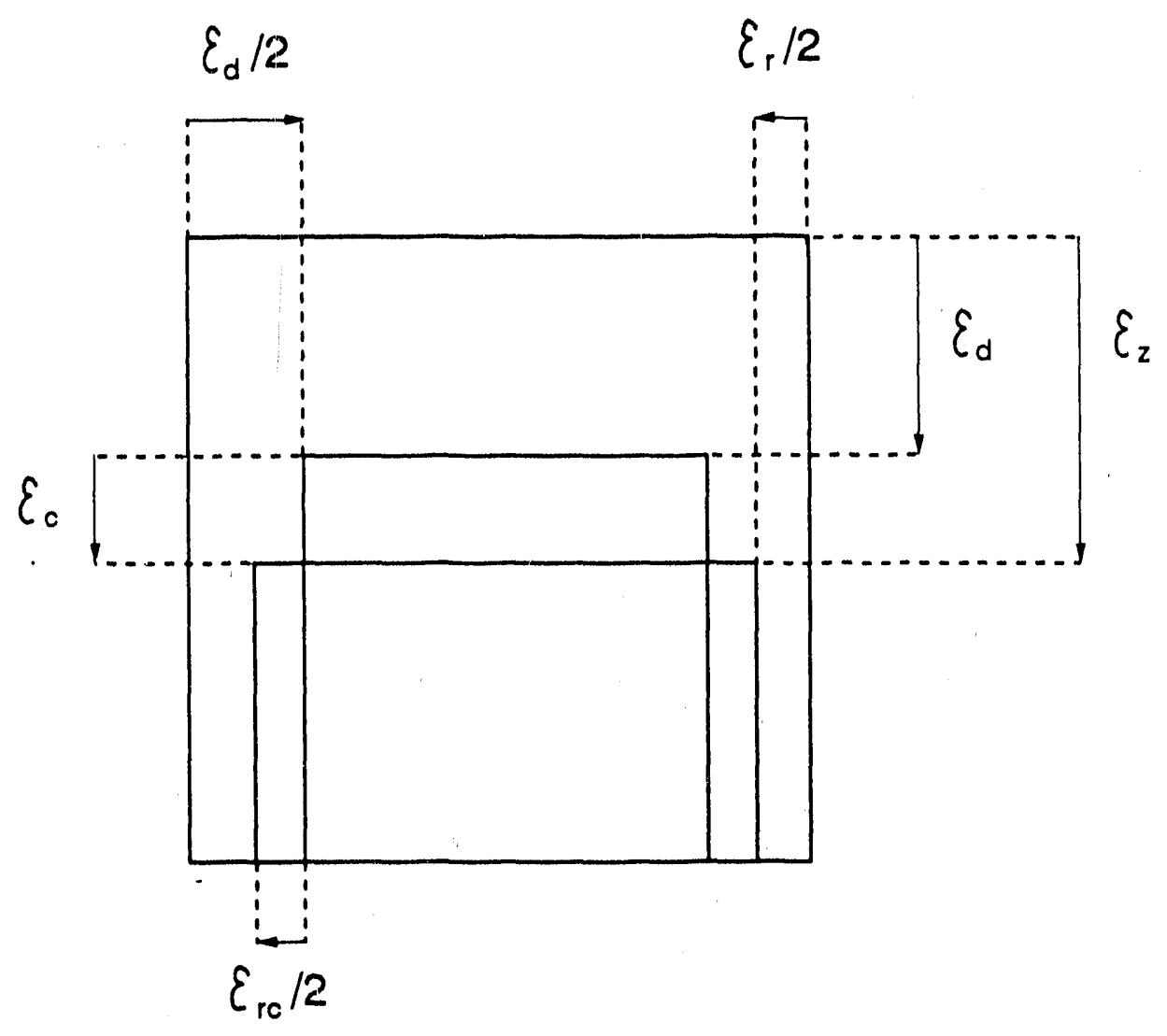

XBL. 886-2027

Fig. 3.7 Various strains in a densifying and creeping compact. 


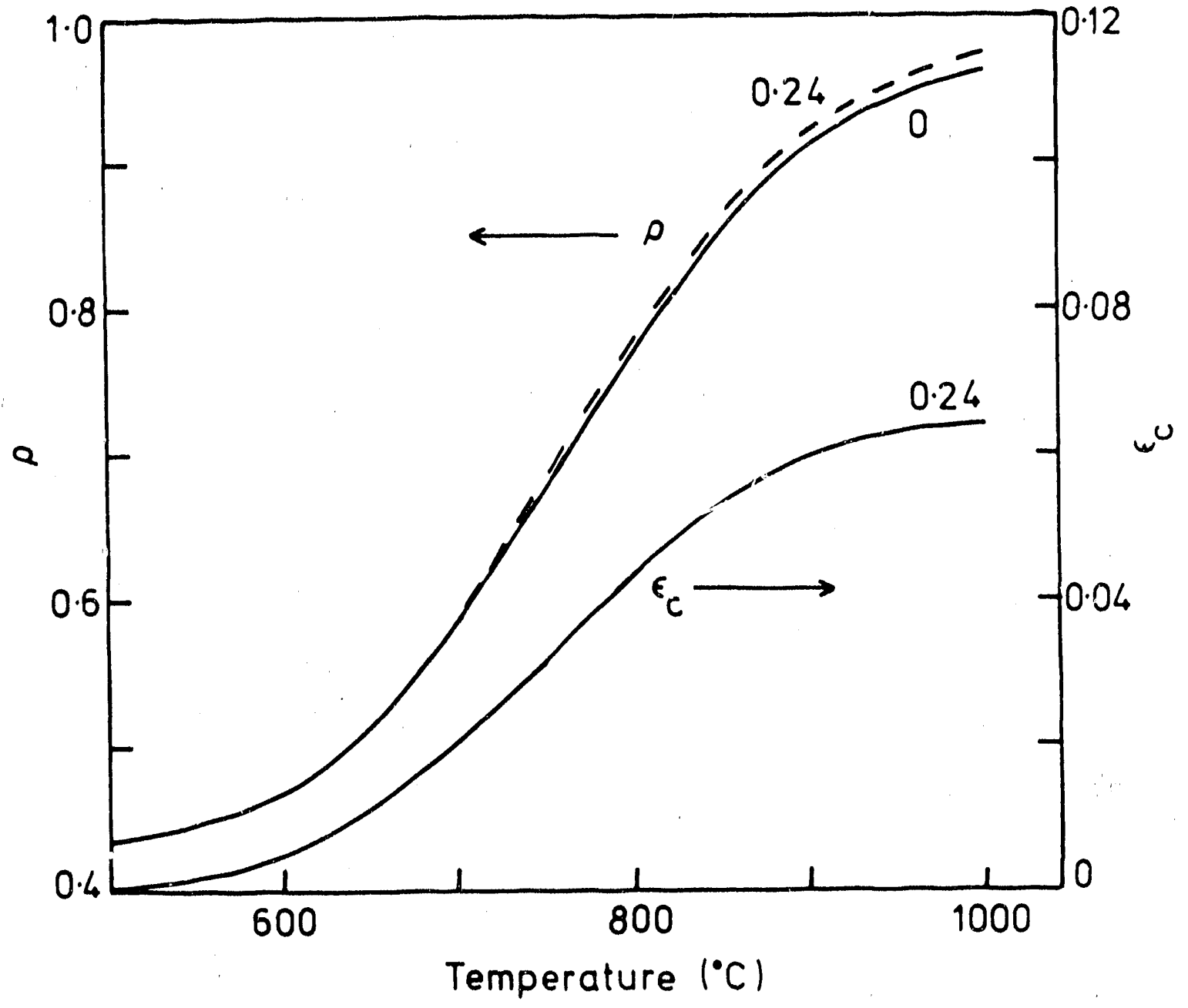

XBL 882-424A

Fig. 3.8 Relative density, $\rho$, and creep strain, $\epsilon_{c}$, calculated from Figs. $3.2 \& 3.3$ and Eqns. 3.6 \& 3.7. 


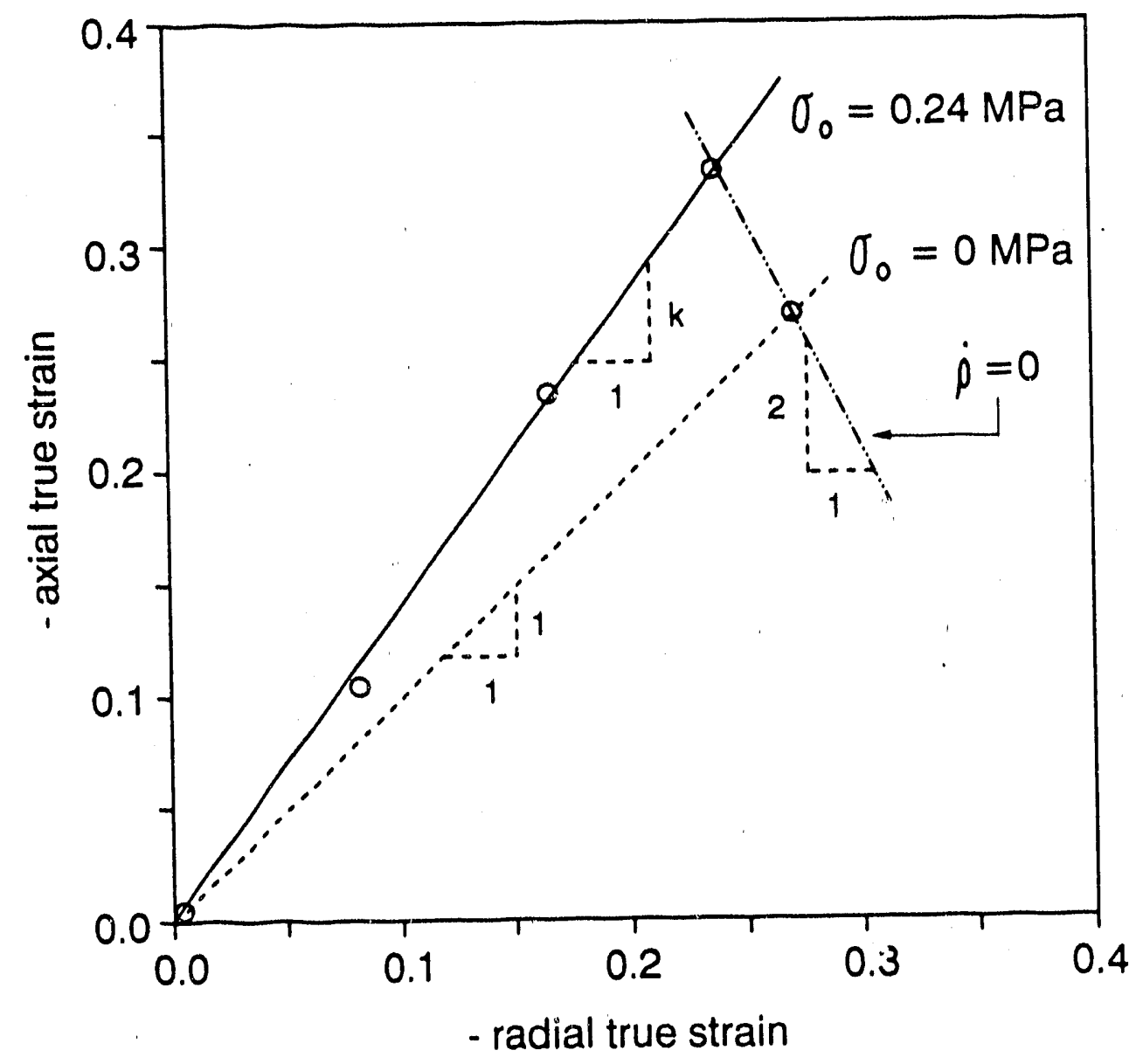

XBL 886-2028

Fig. 3.9a Axial strain versus radial strain for $\mathrm{ZnO}$ with constant density line. 


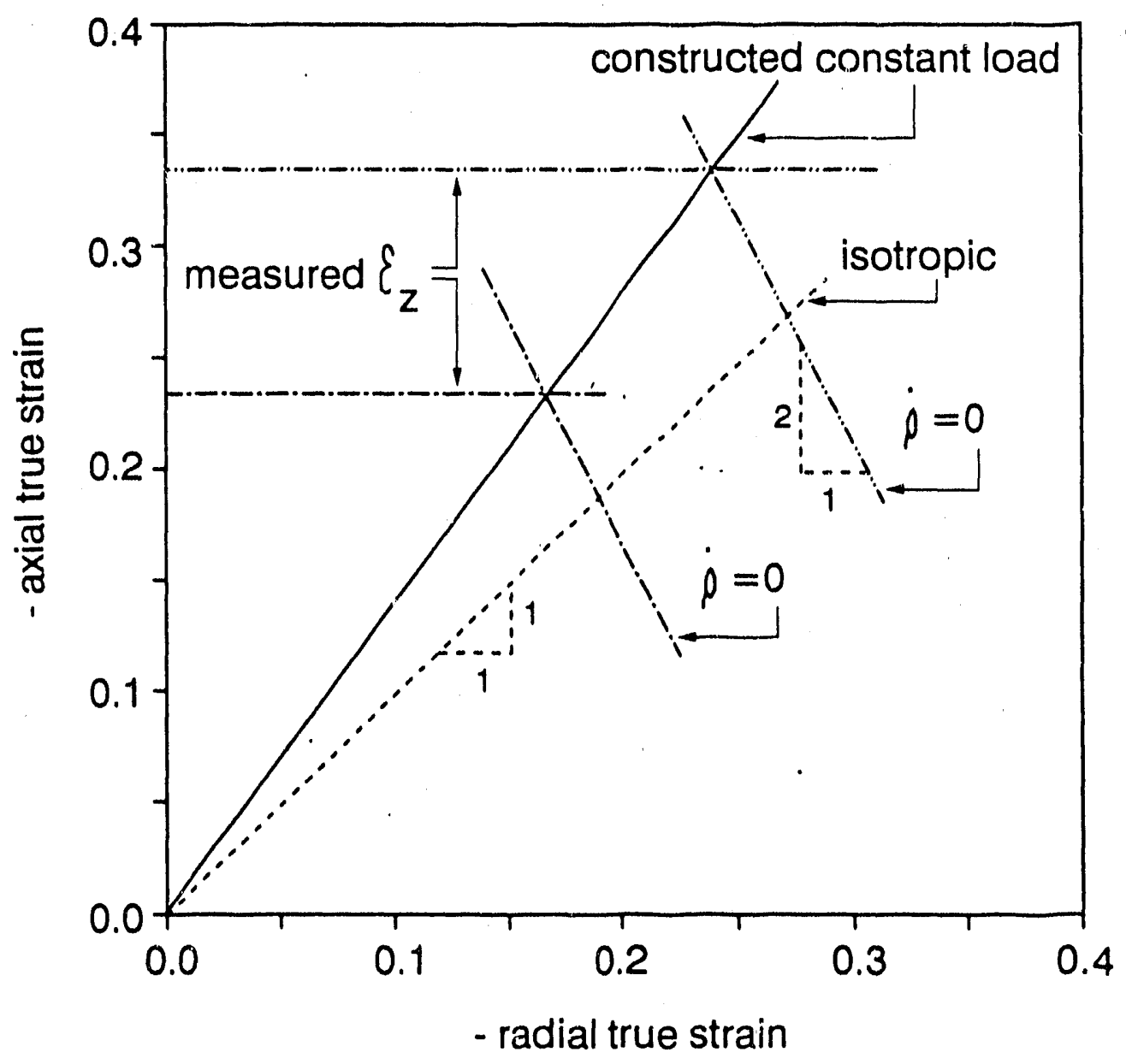

XBL 886-2029

Fig. 3.9b Hypothetical construction of the axial strain versus radial strain from $\epsilon_{z}$ (measured from sample), isotropic line, and constant density line. 


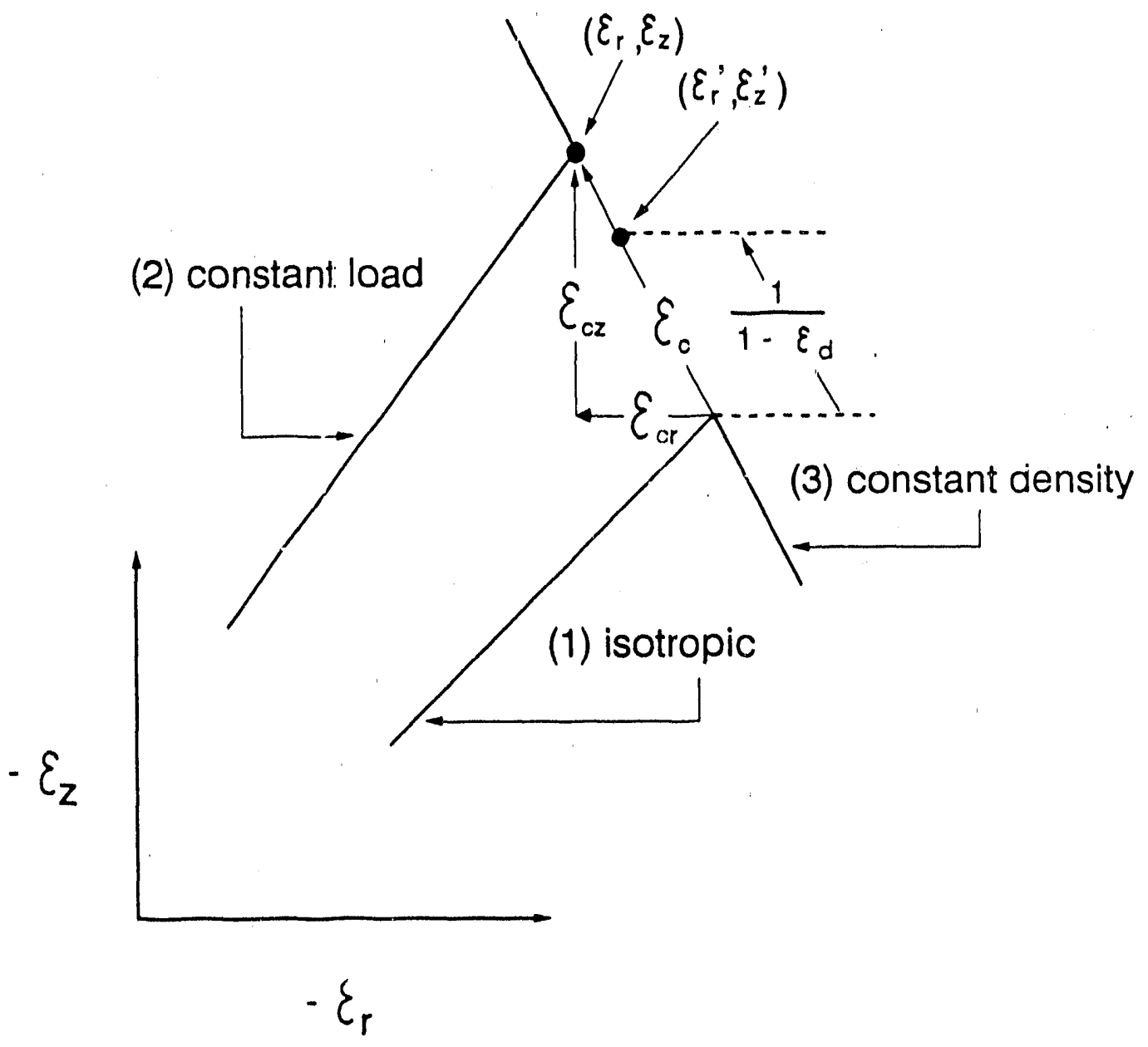

XBL 886-2030

Fig.3.10 Corrections of axial and radial strain from constant load $\left(\epsilon_{z}, \epsilon_{r}\right)$ to constant stress $\left(\epsilon_{z}^{\prime}, \epsilon^{\prime}{ }_{r}\right)$. 


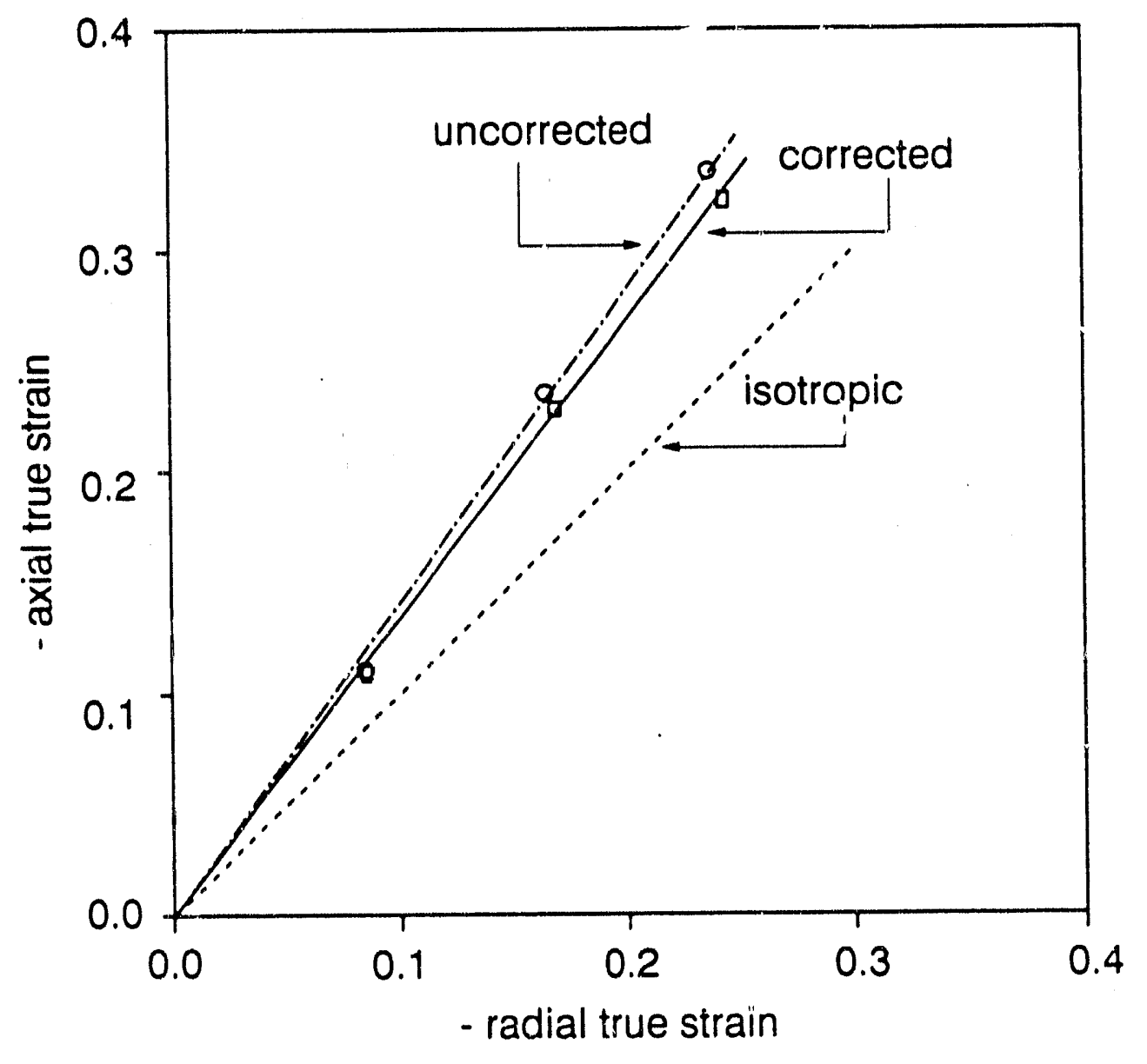

XBL 886-2031

Fig. 3.11 Axial strain versus radial strain for $\mathrm{ZnO}$. Constant load at $7 \mathrm{~N}$ (uncorrected), constant stress at $0.2 \mathrm{MPa}$ (corrected), 0 load (isotropic). 
(a)

(b)

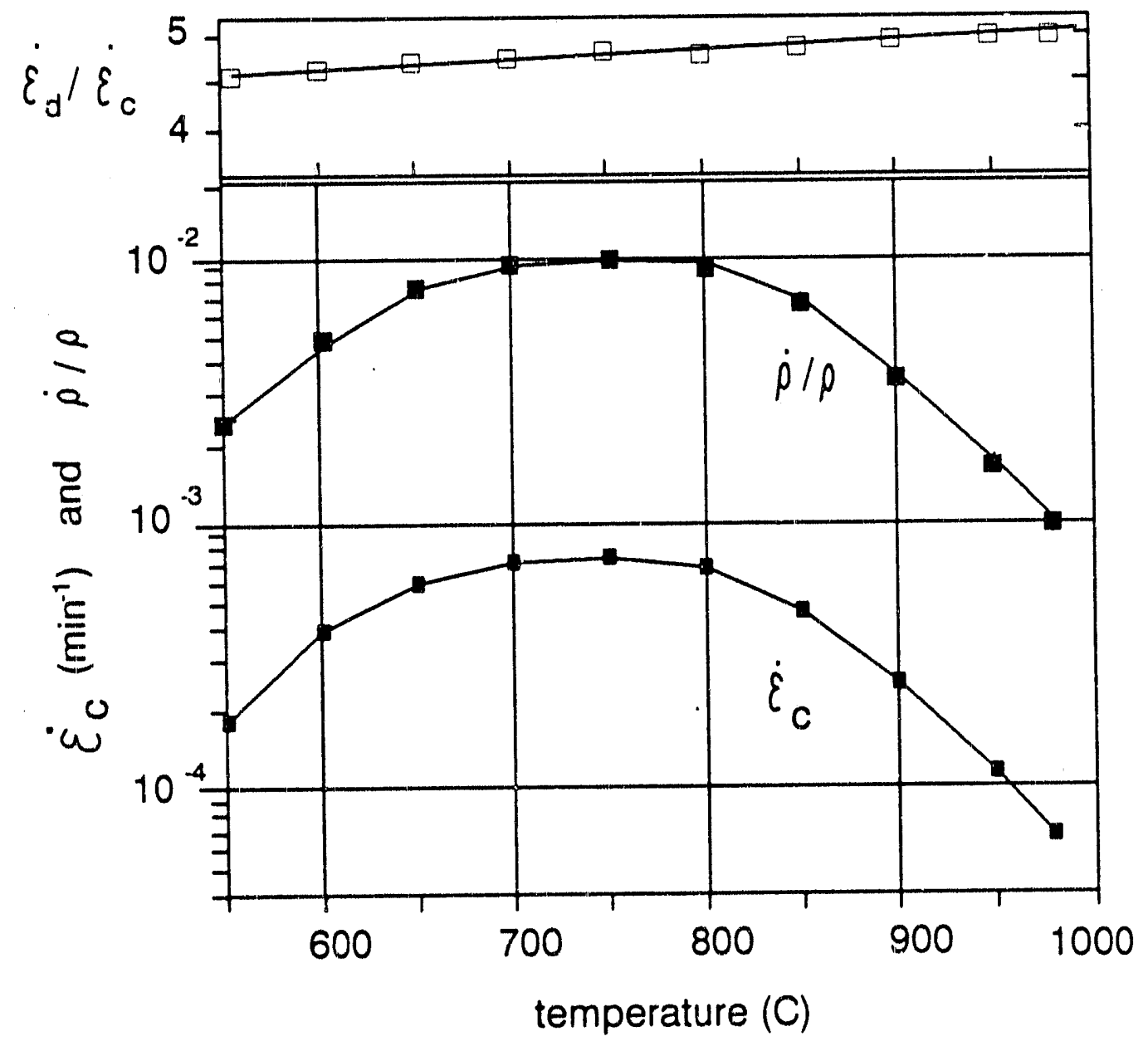

XBL 886-2032

Fig. 3.12 (a) Ratio of the densification to the creep strain rate versus temperature for the uniaxial stress shown. (b) Relative densification strain rate, $\rho / \rho$, and creep strain rate, $\dot{\epsilon}_{c}$, versus temperature at the constant uniaxial stress shown. 


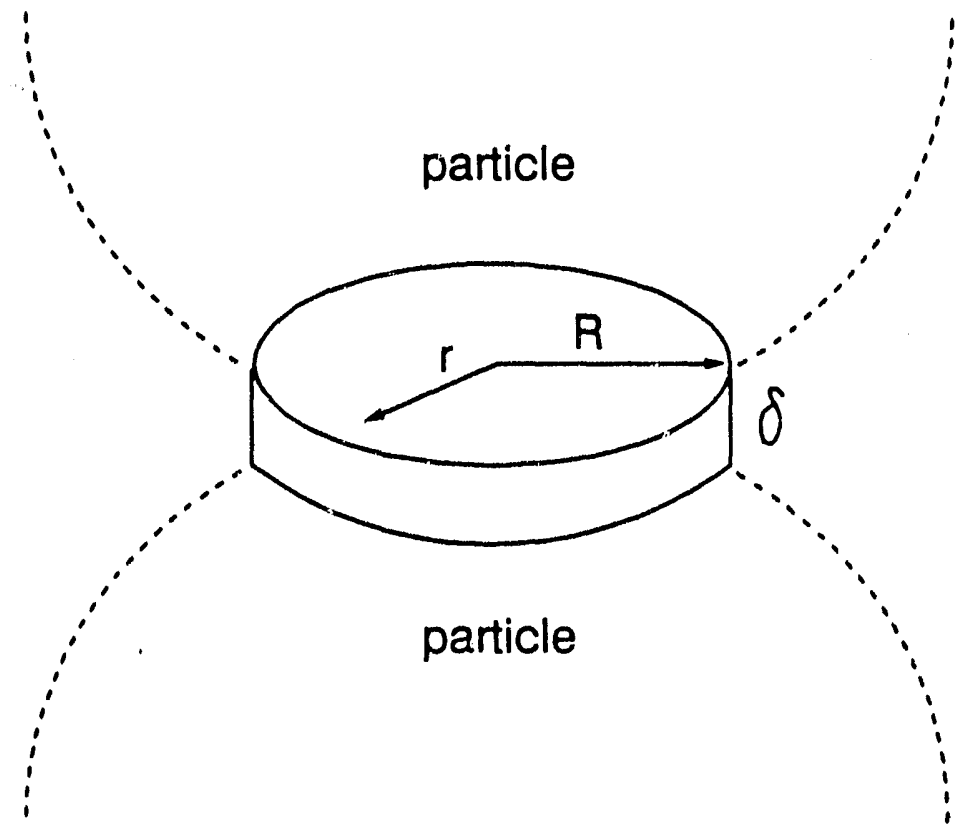

XBL 903-1176

Fig. 3.13 Schematic of two spherical particles with adjoining grain boundary section. 


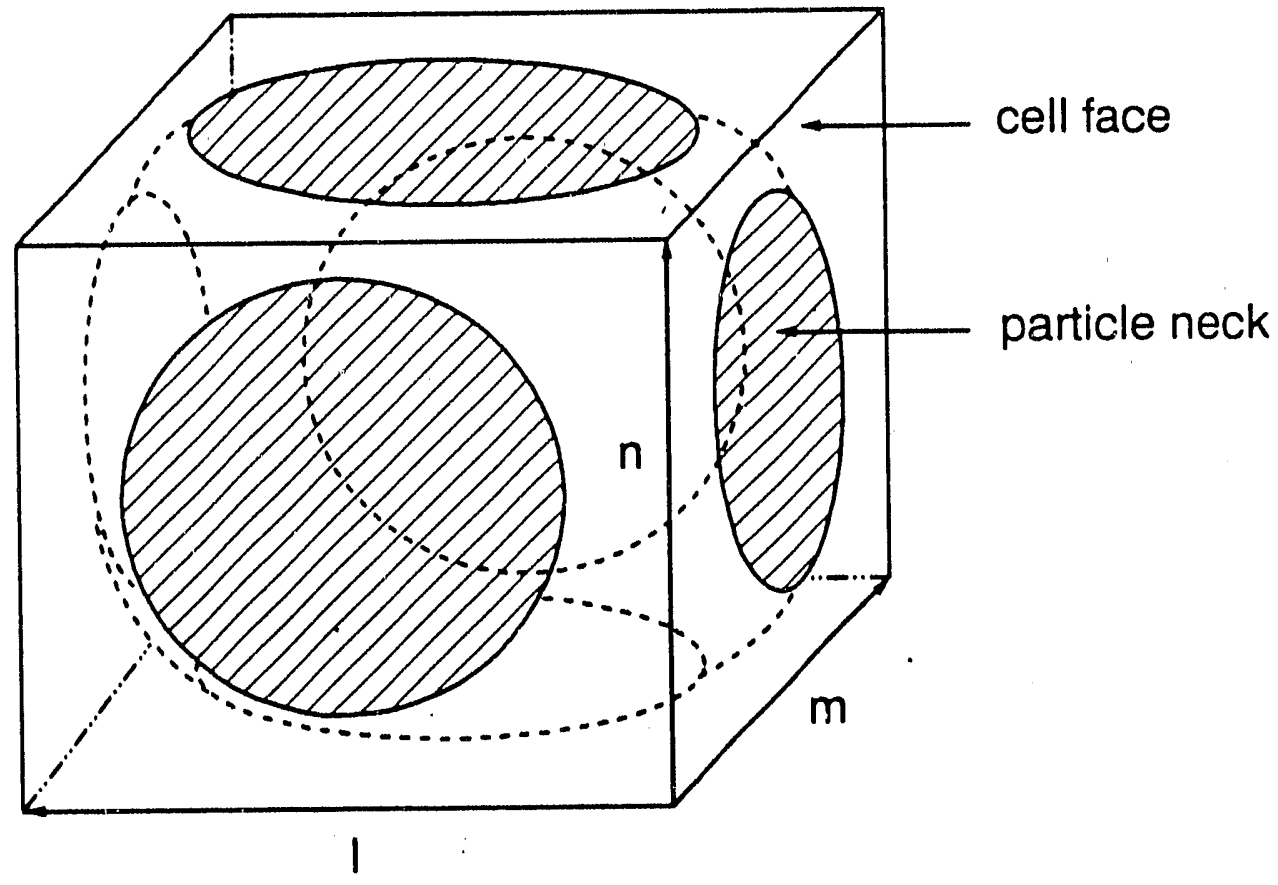

XBL 903-1177

Fig. 3.14 Schematic of cell surrounding spherical particle. 


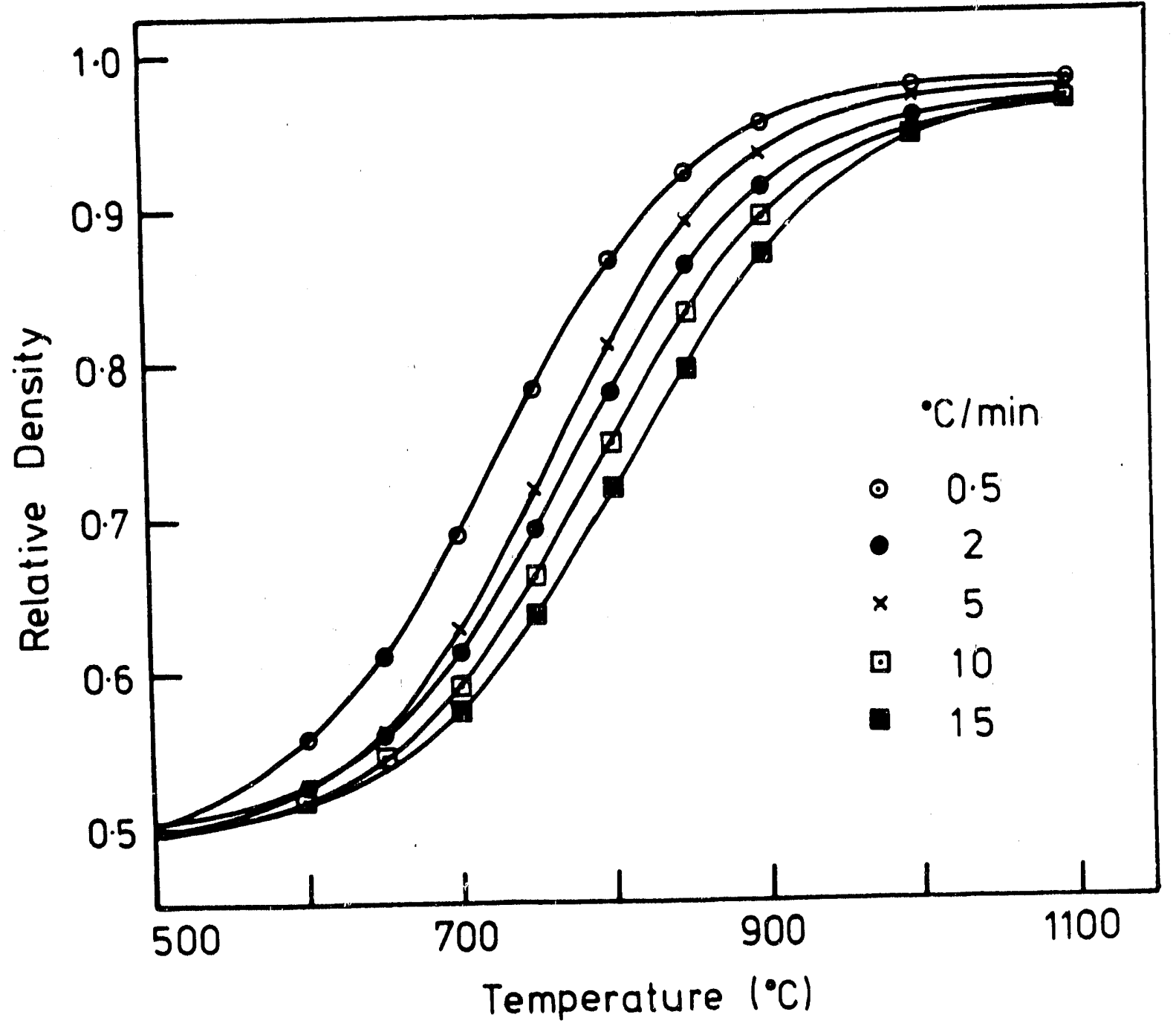

XBL $893-902 A$

Fig. 4.1 Relative density versus temperature for $\mathrm{ZnO}$ powder compacts with the same initial density $(0.50 \pm 0.01)$ and sintered at constant rates of heating shown (in ${ }^{\circ} \mathrm{C} / \mathrm{min}$ ). 


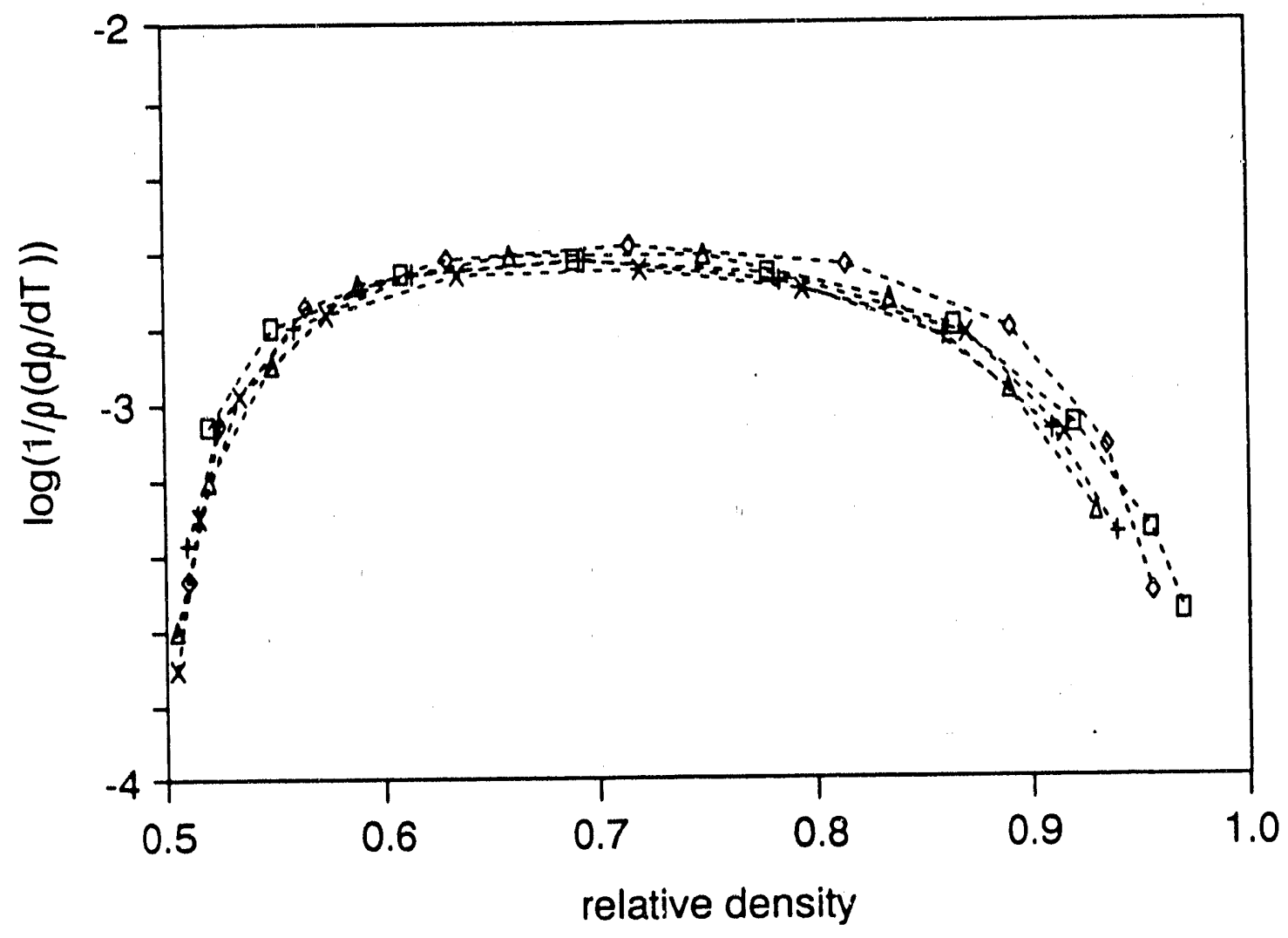

XBL 904-1451

Fig. 4.2 Change in densification strain per unit change in temperature as a function of density calculated from Fig. 4.1. 


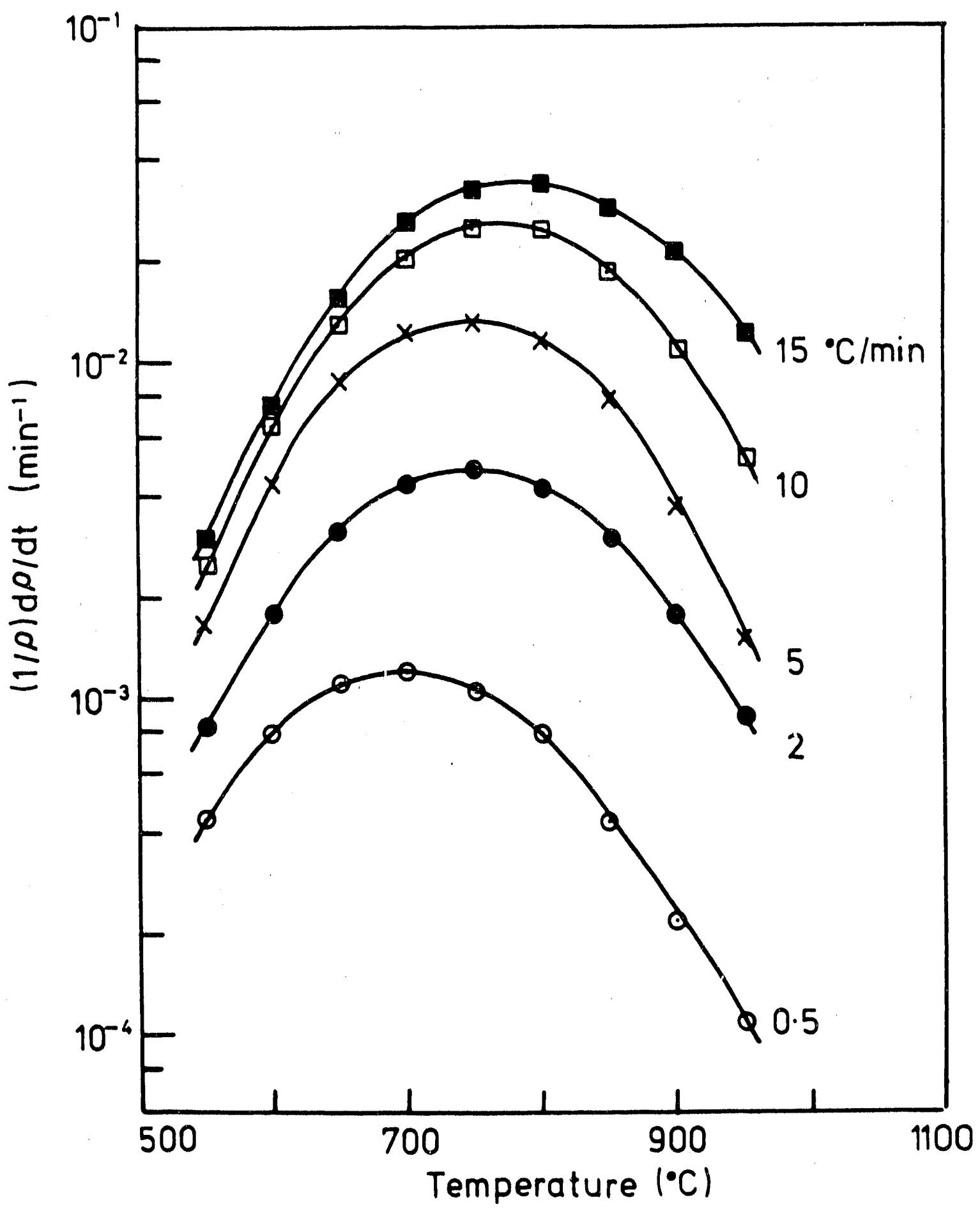

XBL 893-905 A

Fig. 4.3 Densification strain rate versus temperature calculated from Fig. 4.1. 


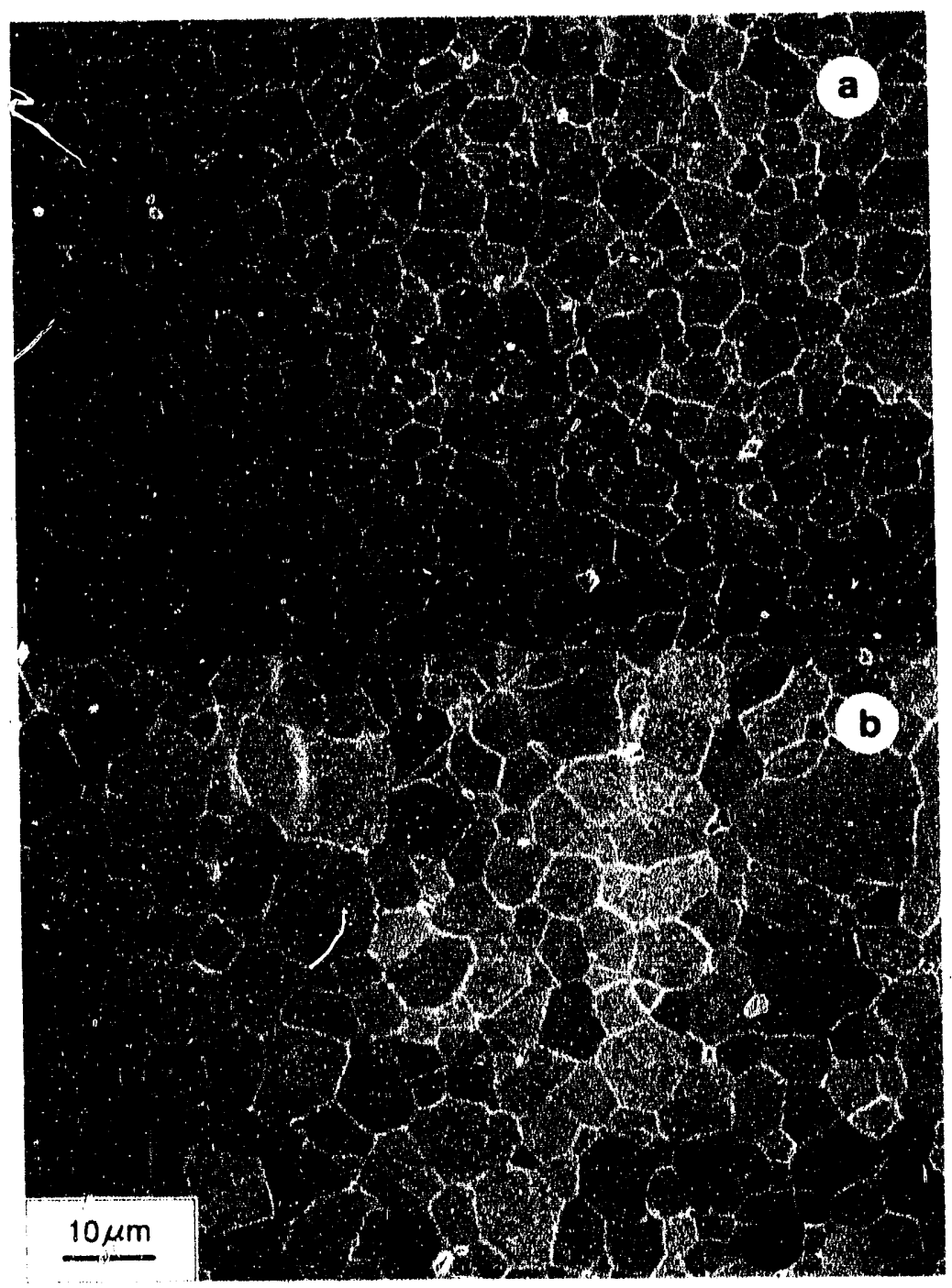

XBB $903.2165 a$

Fig. 4.4 Scanning electron micrographs of polished and eiched surfaces of samples sintered to $1100^{\circ} \mathrm{C}$ at constant heating rates of (a) $5^{\circ} \mathrm{C} / \mathrm{min}$. and (b) $0.5^{\circ} \mathrm{C} / \mathrm{min}$. 


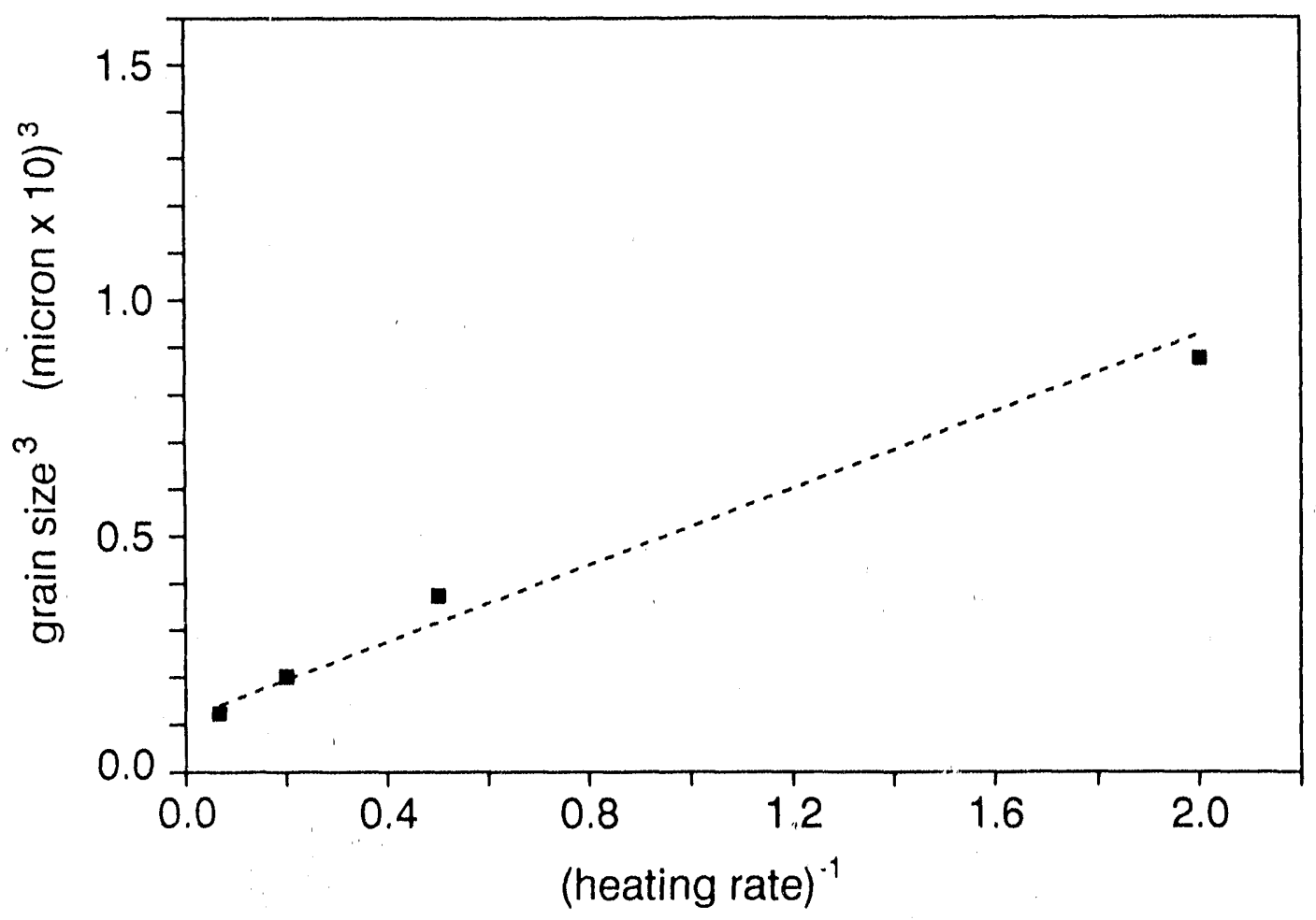

XBL 903-1175

Fig. 4.5 Cube of the final grain size from various heating rates versus the inverse of the heating rate, $\alpha^{-1}$. (correlation coefficient $=0.995$ ) 


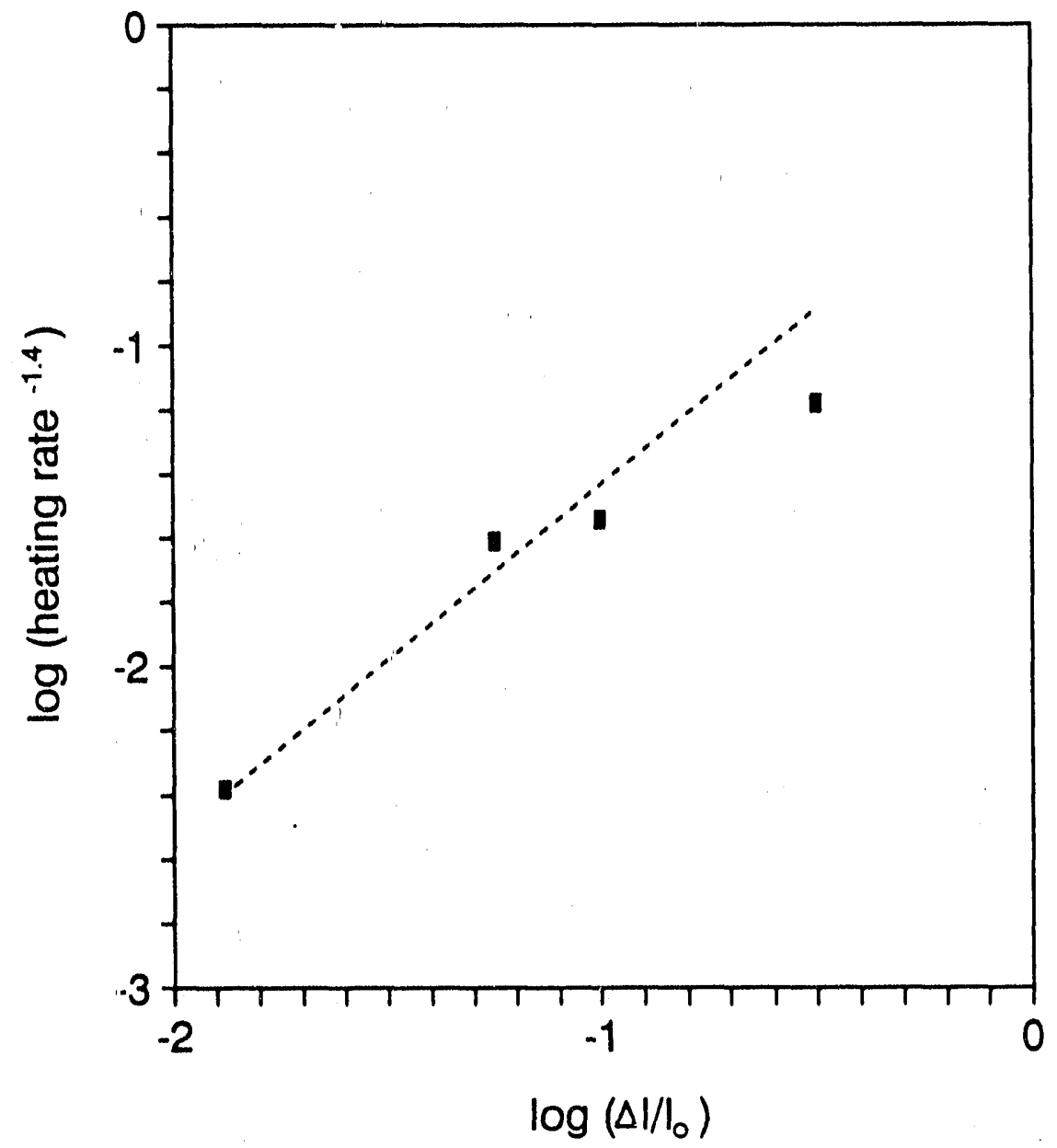

XBL $904-1450$

Fig. 4.6 Log of the heating rate $\mathrm{e}^{-1.4}$ versus $\log$ of the incremental shrinkage at a constant temperature of $1160^{\circ} \mathrm{C}$ [Barringer et al 1984]. 


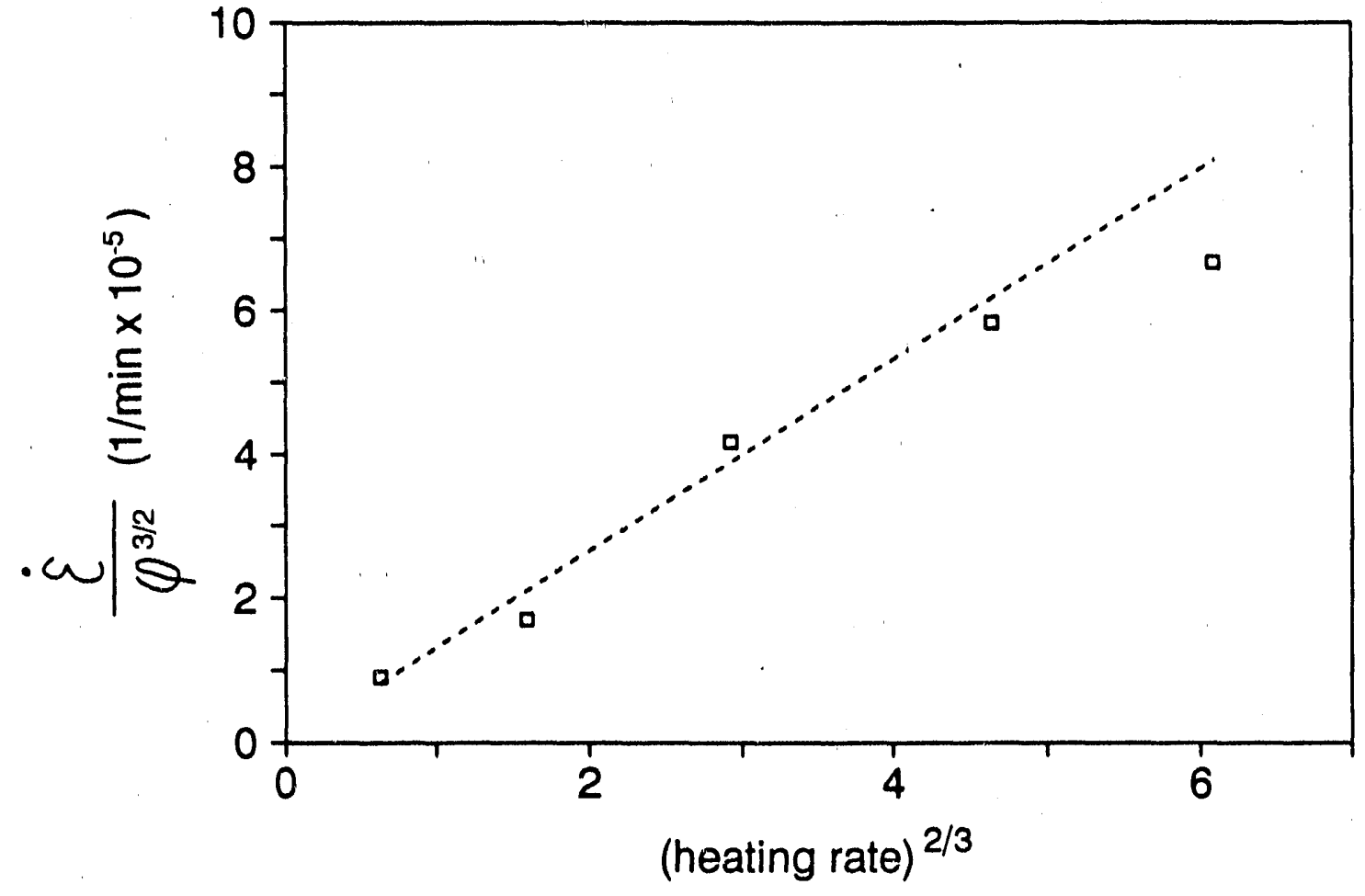

XEL,, 03.1173

Fig. 4.7 Densification strain rate divided by $\phi^{3 / 2}$ versus heating rate to the $2 / 3$ power, evaluated at $600^{\circ} \mathrm{C}$. 


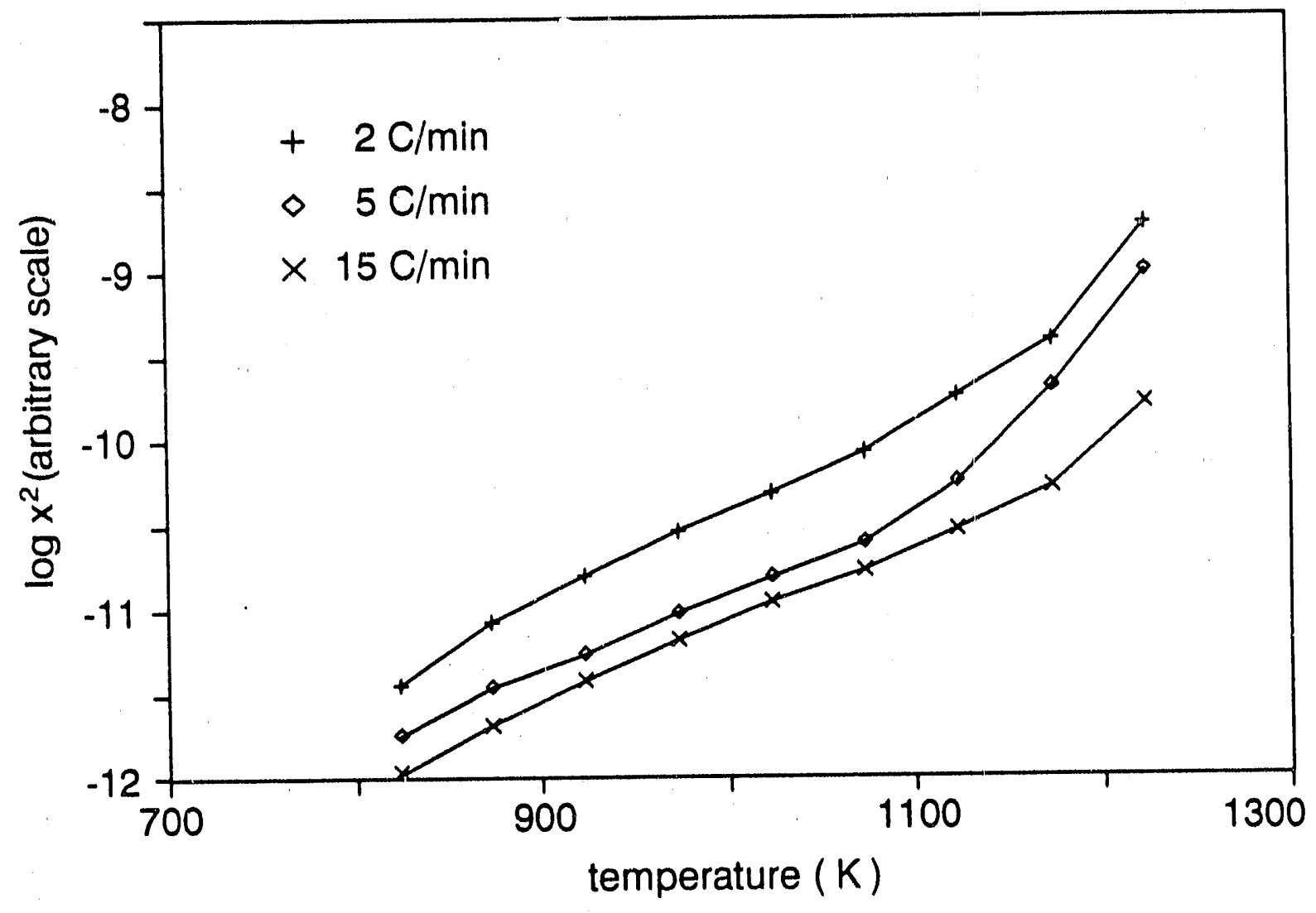

XBL 903-1174

Fig. 4.8 The log of the square of average interpore spacing versus temperature. 


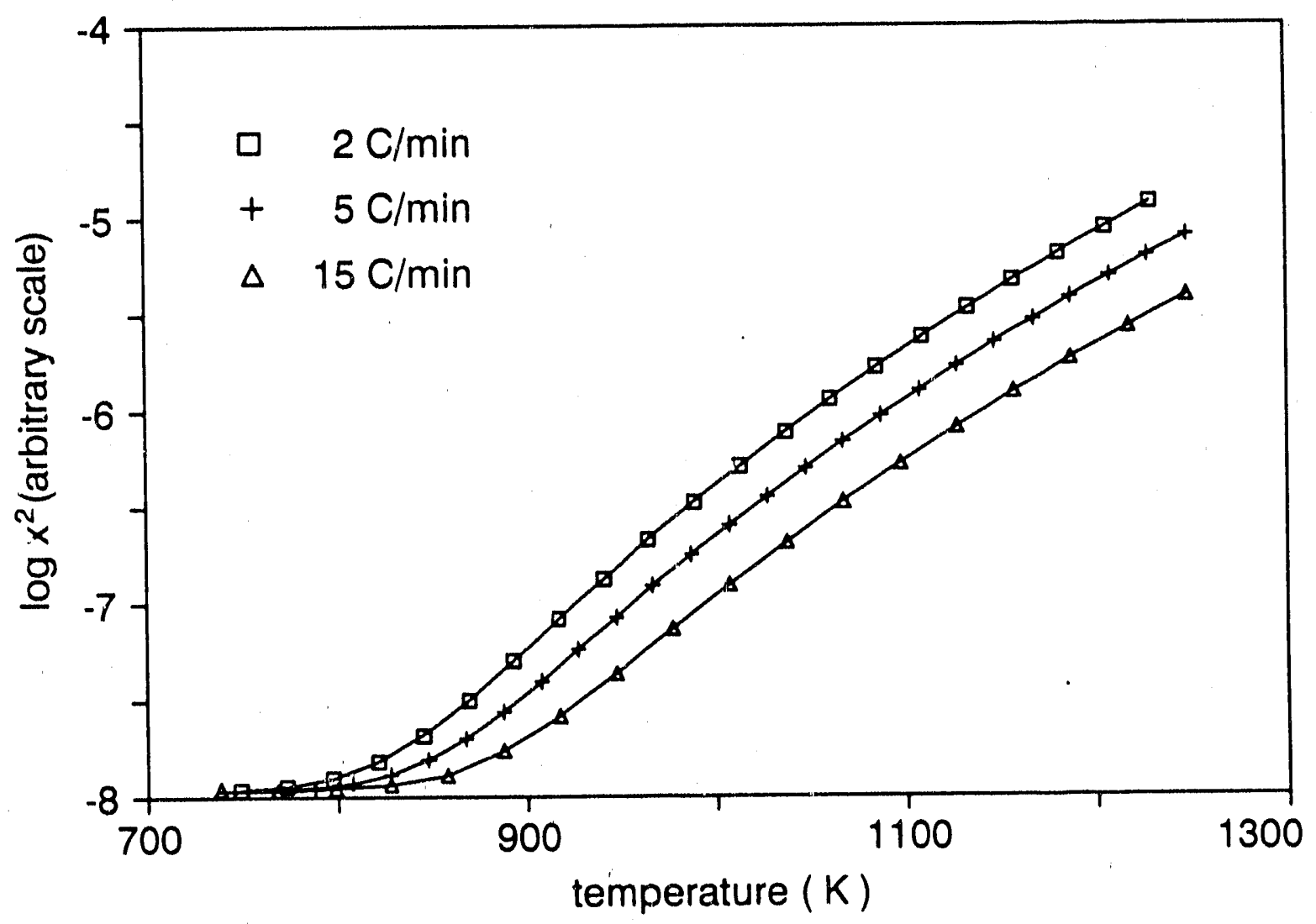

XBL. 903-1172

Fig. 4.9a $\log$ of the square of the mean interpore spacing versus temperature calculated from Eqn. 4.5 with heating rates of $15^{\circ} \mathrm{C} / \mathrm{min}, 5^{\circ} \mathrm{C} / \mathrm{min}$, and $2^{\circ} \mathrm{C} / \mathrm{min} . \mathrm{x}_{\mathrm{o}}{ }^{2}$ and prefactors were chosen such that $\mathrm{x} \approx 60 \mathrm{x}_{\mathrm{O}}$ at $1100^{\circ} \mathrm{C}$ for the heating rate at $5^{\circ} \mathrm{C} / \mathrm{min}$; coarsening activation energy $=50 \mathrm{kcal} / \mathrm{mole}$. 


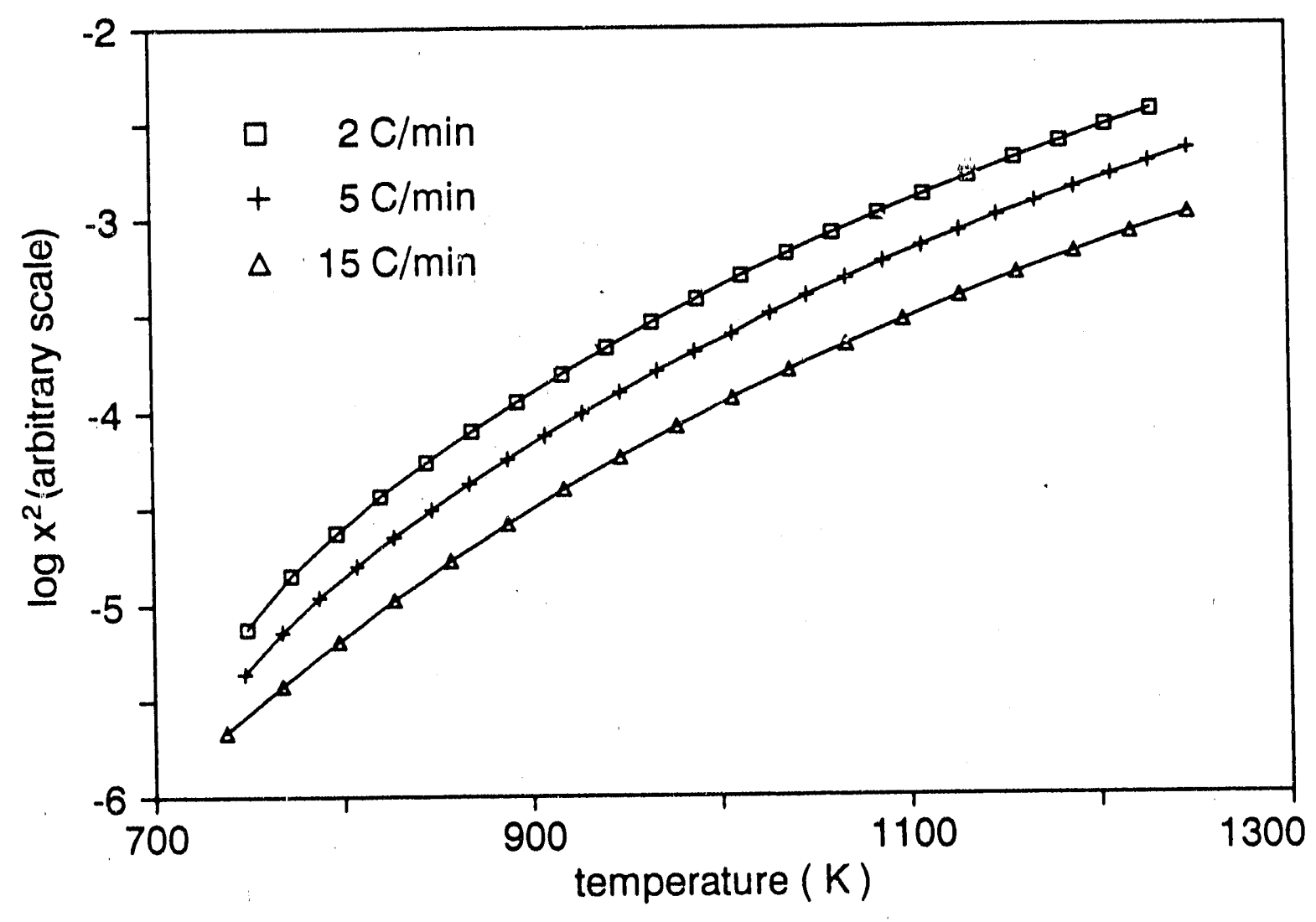

XBL. 903-1171

Fig. 4.9b Log of the square of the mean interpore spacing versus temperature calculated from Eqn. 4.5 with heating rates of $15^{\circ} \mathrm{C} / \mathrm{min}, 5^{\circ} \mathrm{C} / \mathrm{min}$, and $2{ }^{\circ} \mathrm{C} / \mathrm{min} . \mathrm{x}_{\mathrm{O}}{ }^{2}$ and prefactors were chosen such that $\mathrm{x} \approx 60 \mathrm{x}_{\mathrm{O}}$ at $1100^{\circ} \mathrm{C}$ for the heating rate at $5^{\circ} \mathrm{C} / \mathrm{min}$; coarsening activation energy $=30 \mathrm{kcal} / \mathrm{mole}$. 


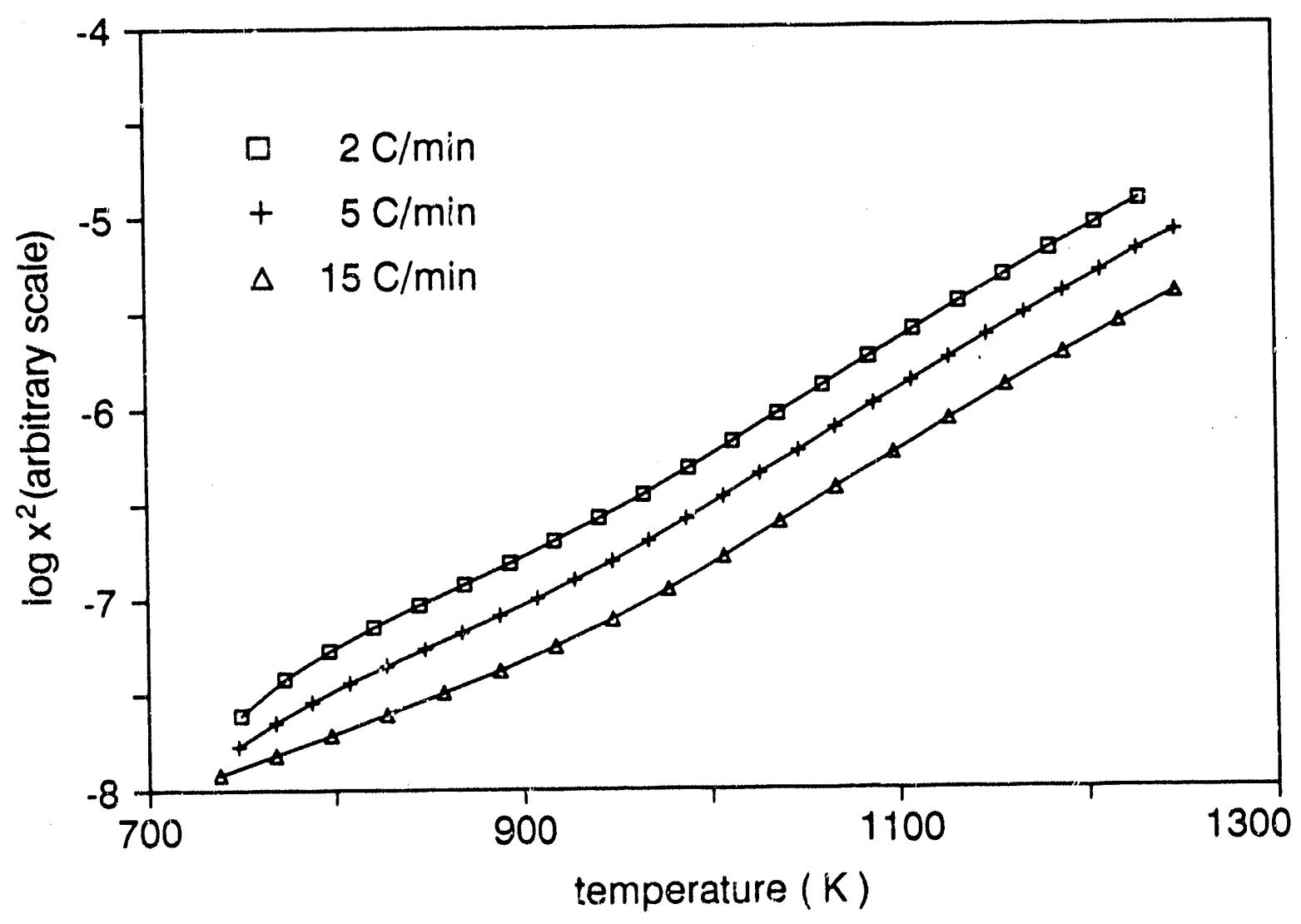

XBL 903-1170

Fig. 4.10 Log of the square of the mean interpore spacing versus temperature calculated from Eqn. 4.10 with heating rates of $15^{\circ} \mathrm{C} / \mathrm{min}, 5^{\circ} \mathrm{C} / \mathrm{min}$, and $2^{\circ} \mathrm{C} / \mathrm{min}$ using activation energies for densification as $50 \mathrm{kcal} / \mathrm{mole}$, and activation energies for coarsening as $50 \mathrm{kcal} / \mathrm{mole}$ and $15 \mathrm{kcal} / \mathrm{mole} . \mathrm{x}_{\mathrm{O}}$ and prefactors were chosen such that $\mathrm{x} \approx 60 \mathrm{x}_{0}$ at $1100^{\circ} \mathrm{C}$ and $\approx 2 \mathrm{x}_{0}$ at $550^{\circ} \mathrm{C}$ for the heating rate $5^{\circ} \mathrm{C} / \mathrm{min}$. 


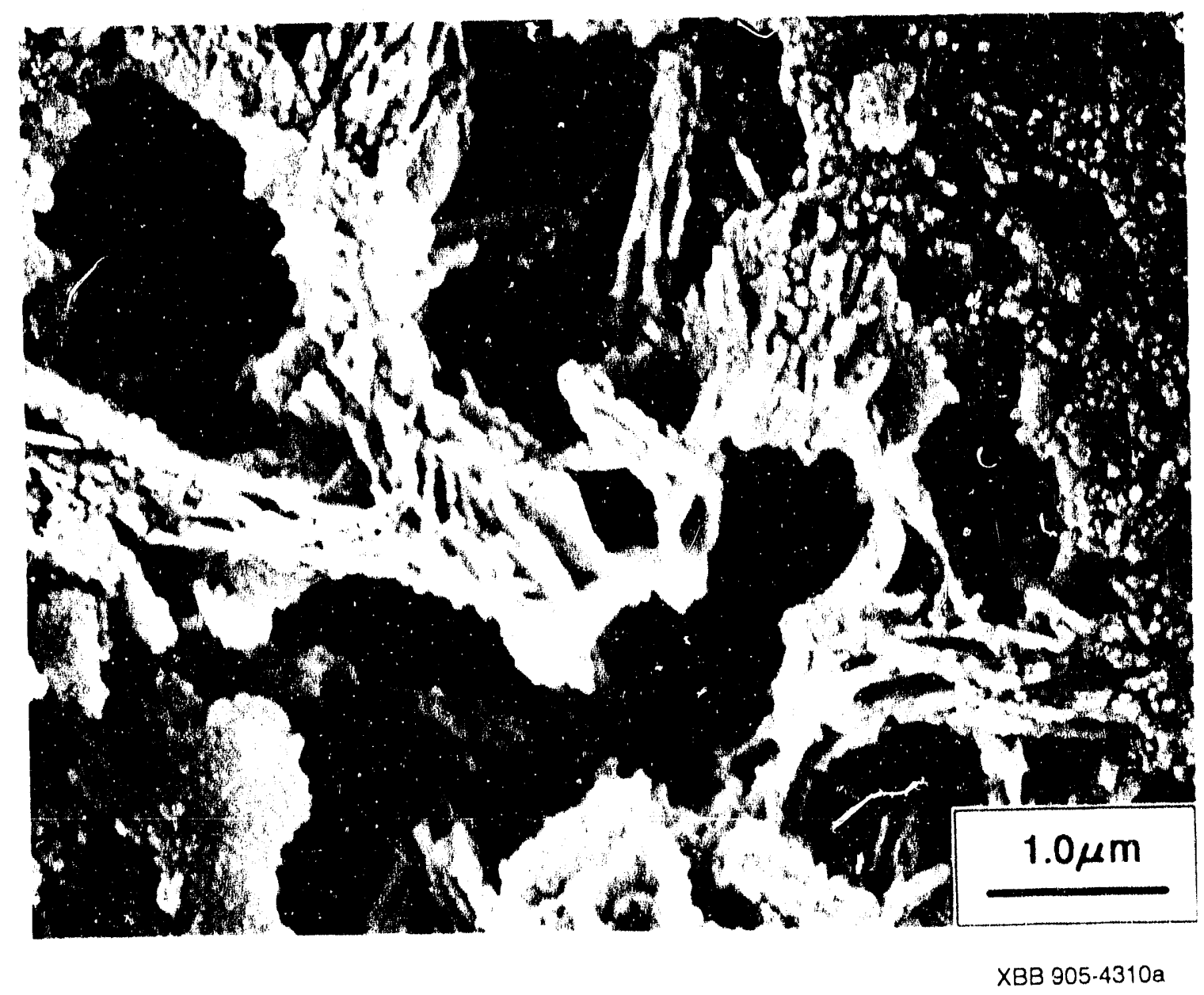

Fig. 5.1 Scanning electron micrograph of a fracture surface of a $\mathrm{MgO}$ initial compact. 


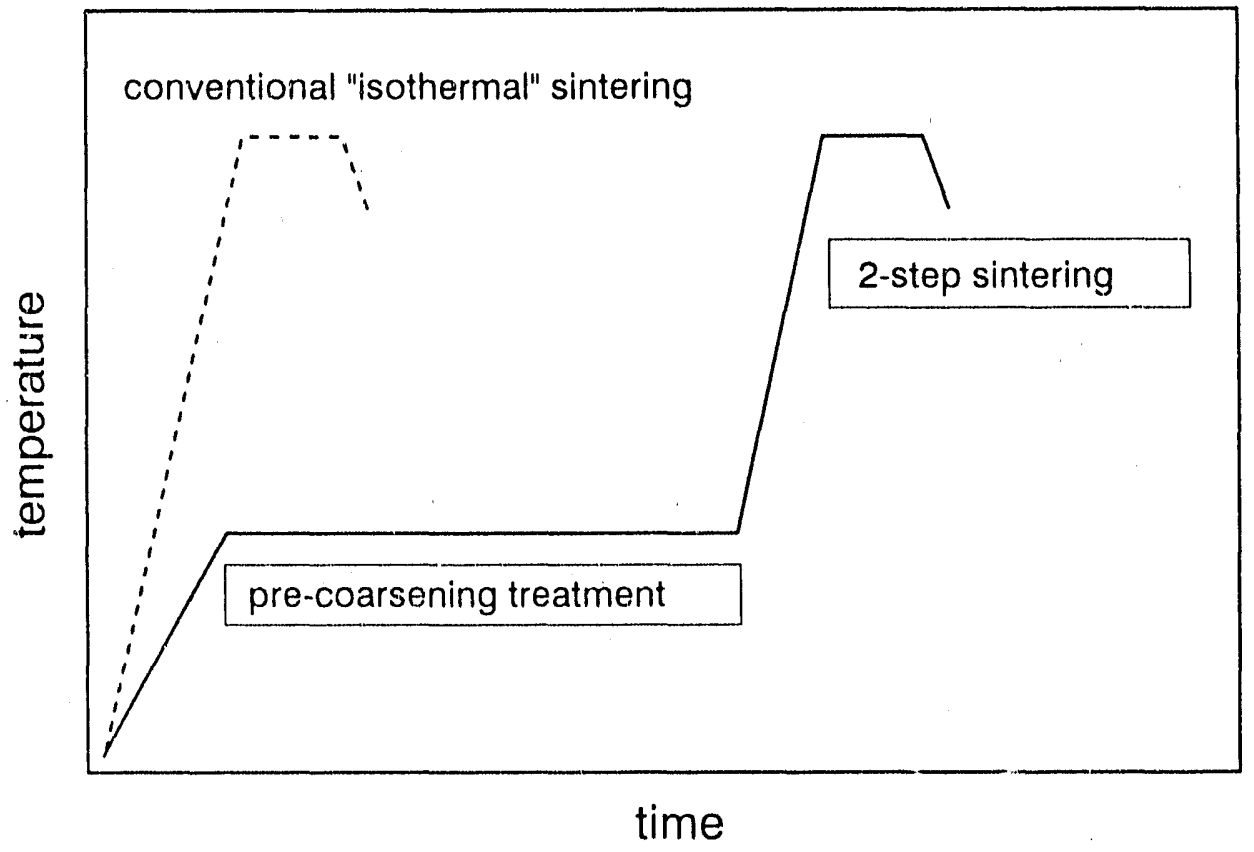

XBL 905-1899

Fig. 5.2 Schematic time-temperature heat' 'g schedules of conventional "isothermal" sintering and of 2-step sintering. 


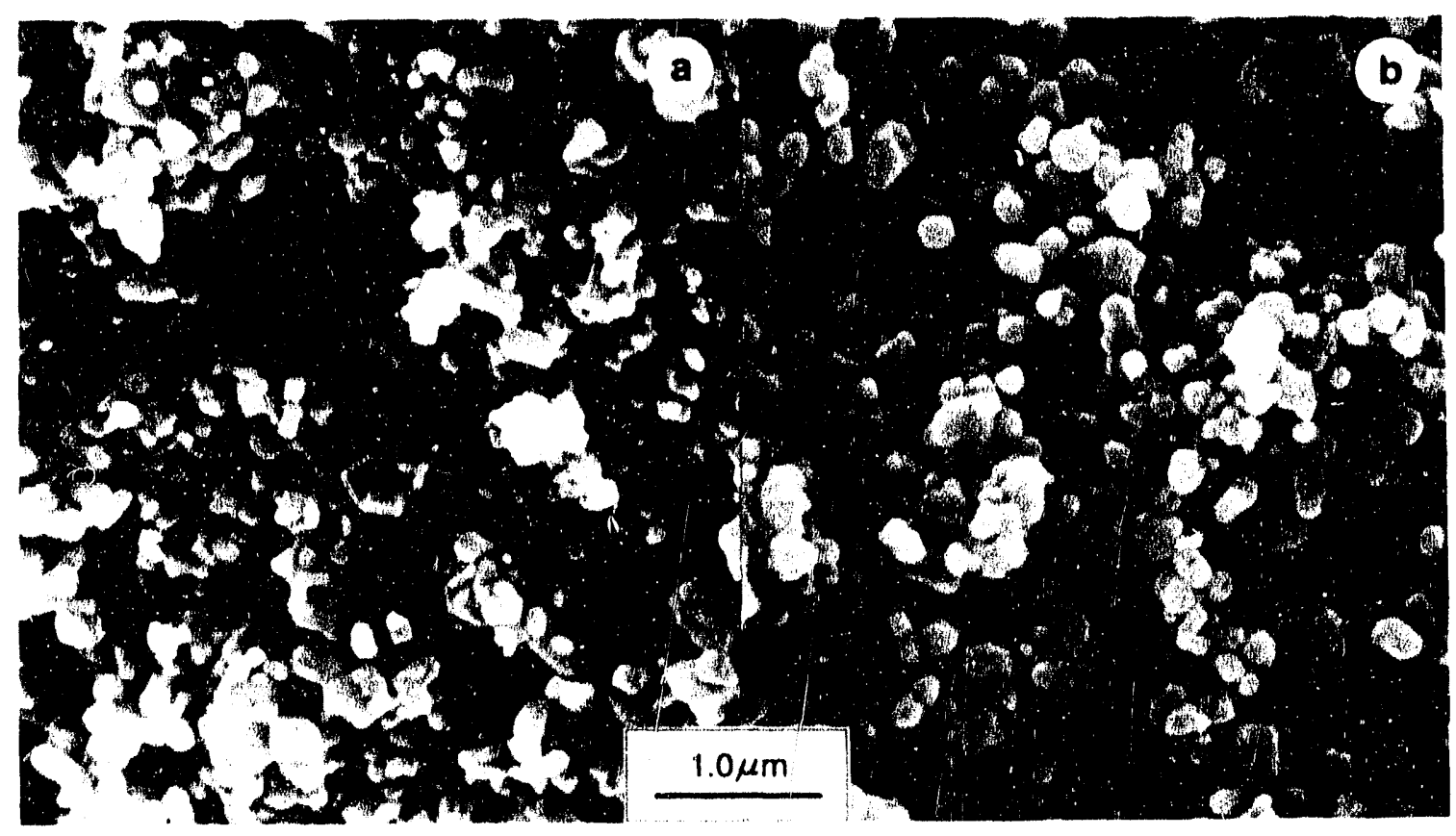

XBB 905-4309a

Pig. 5.3 Scanning electron micrographs of fracture surfaces of $\mathrm{ZnO}$ compacts. (a) Green compact of as-received $\mathrm{ZnO}$, relative density of 0.49 . (b) Compact after pre-coarsening treatment to a relative density of 0.50 . 


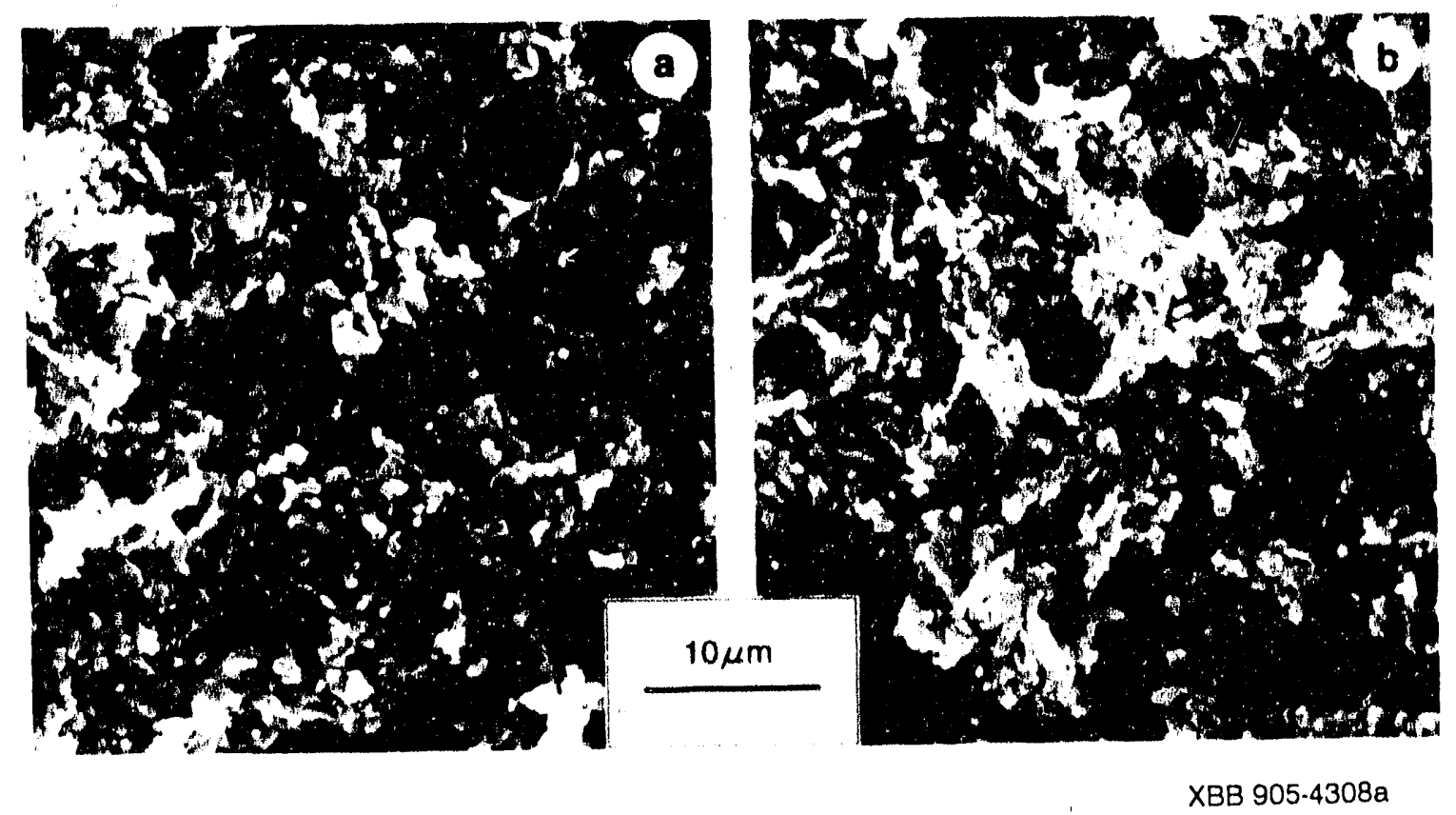

Fig. 5.4 Scanning electron micrographs of fracture surfaces of $\mathrm{MgO}$ compacts sintered to a relative density of 0.60 . (a) After isothermal at $1500^{\circ} \mathrm{C}$. (b) After isothermal sintering at $1250^{\circ} \mathrm{C}$. 


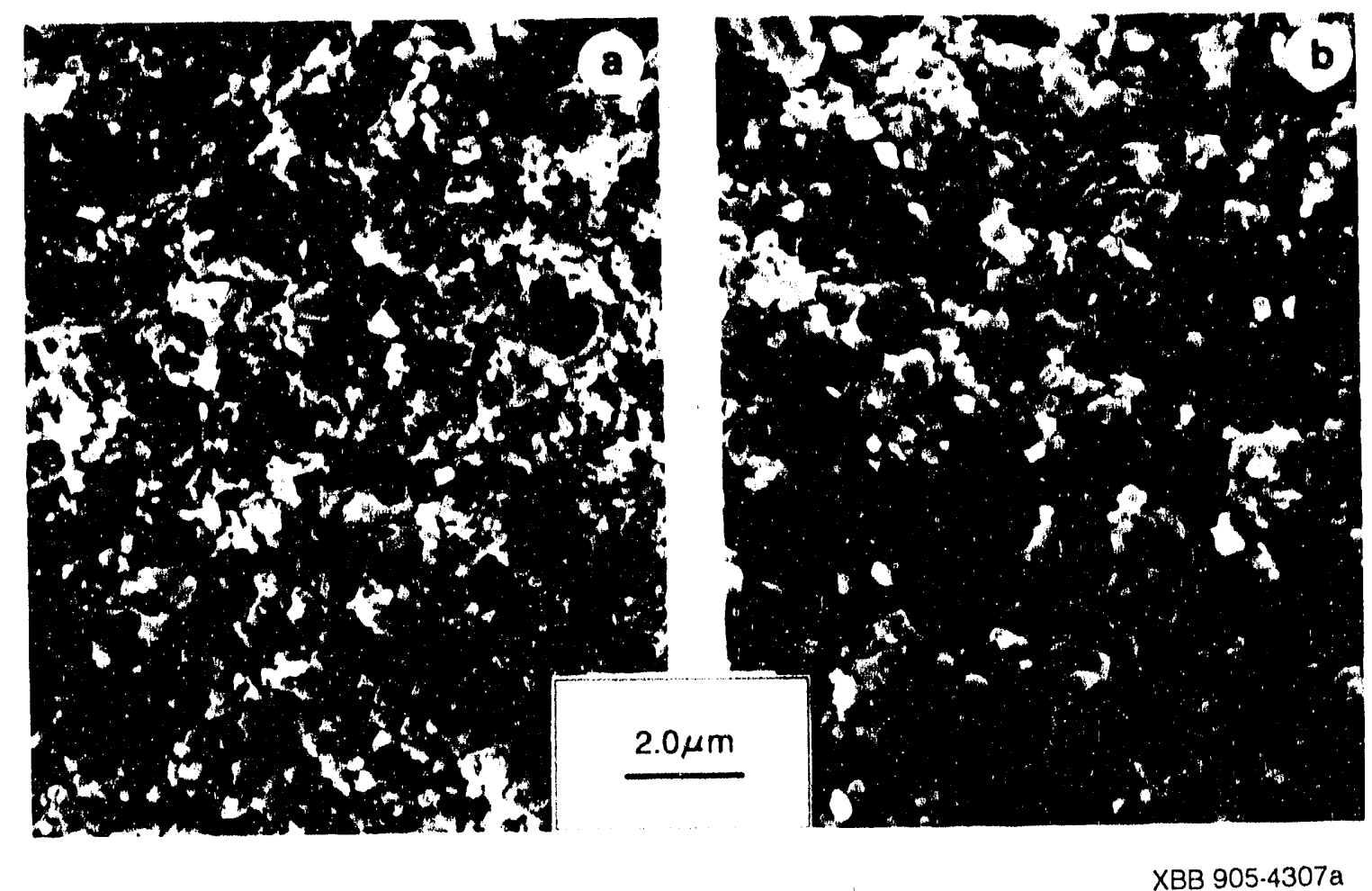

Fig. 5.5 Scanning electron micrographs of fracture surfaces of $\mathrm{MgO}$ compacts sintered to a relative density of 0.70 . (a) After isothermal sintering at $1500^{\circ} \mathrm{C}$. (b) After isothermal sintering at $1250^{\circ} \mathrm{C}$. 


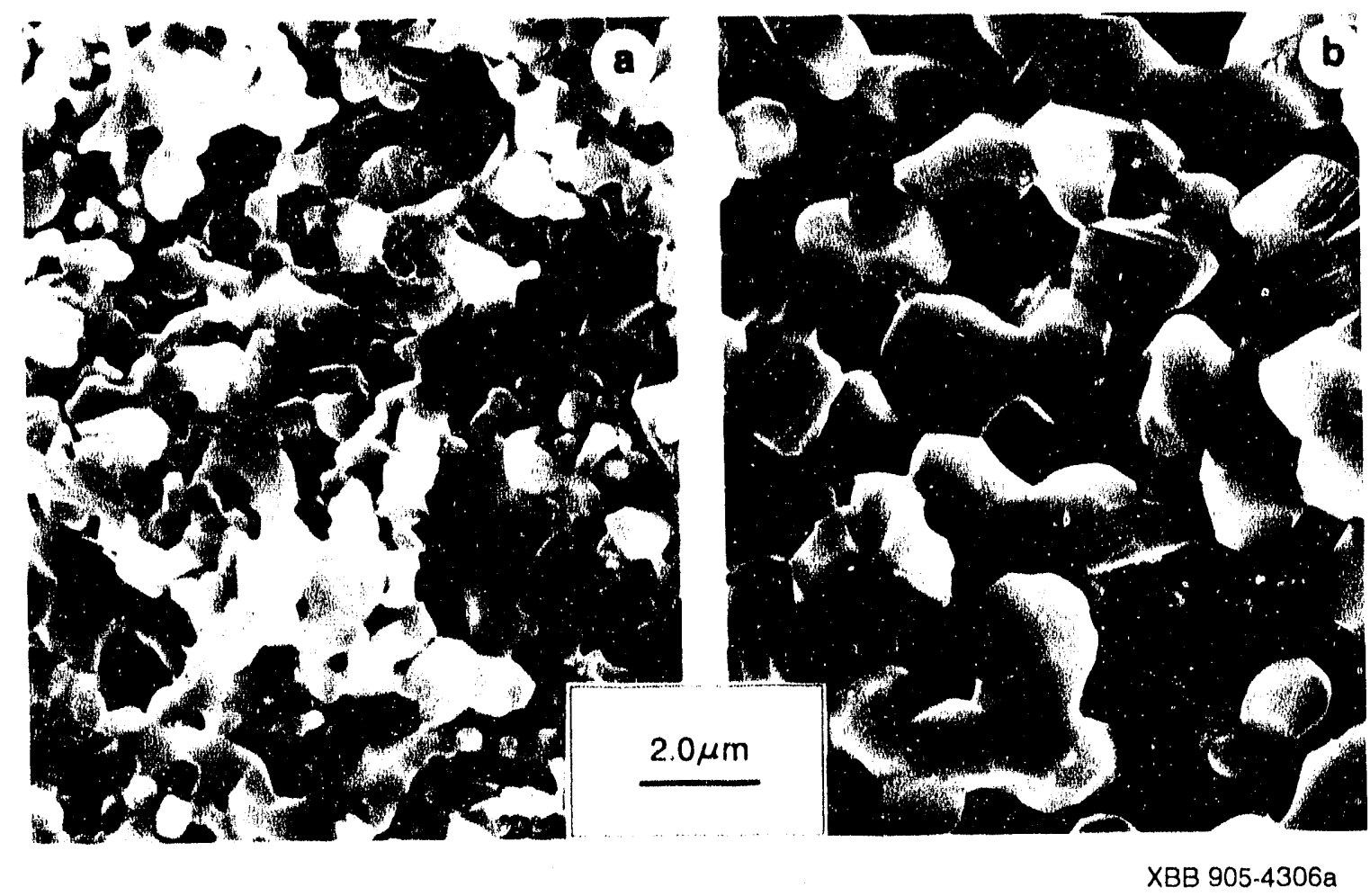

Fig. 5.6 Scanning electron micrographs of fracture surfaces of $\mathrm{MgO}$ compacts sintered to a relative density of 0.80 . (a) After isothermal sintering at $1500^{\circ} \mathrm{C}$. (b) After isothermal sintering at $1250^{\circ} \mathrm{C}$. 

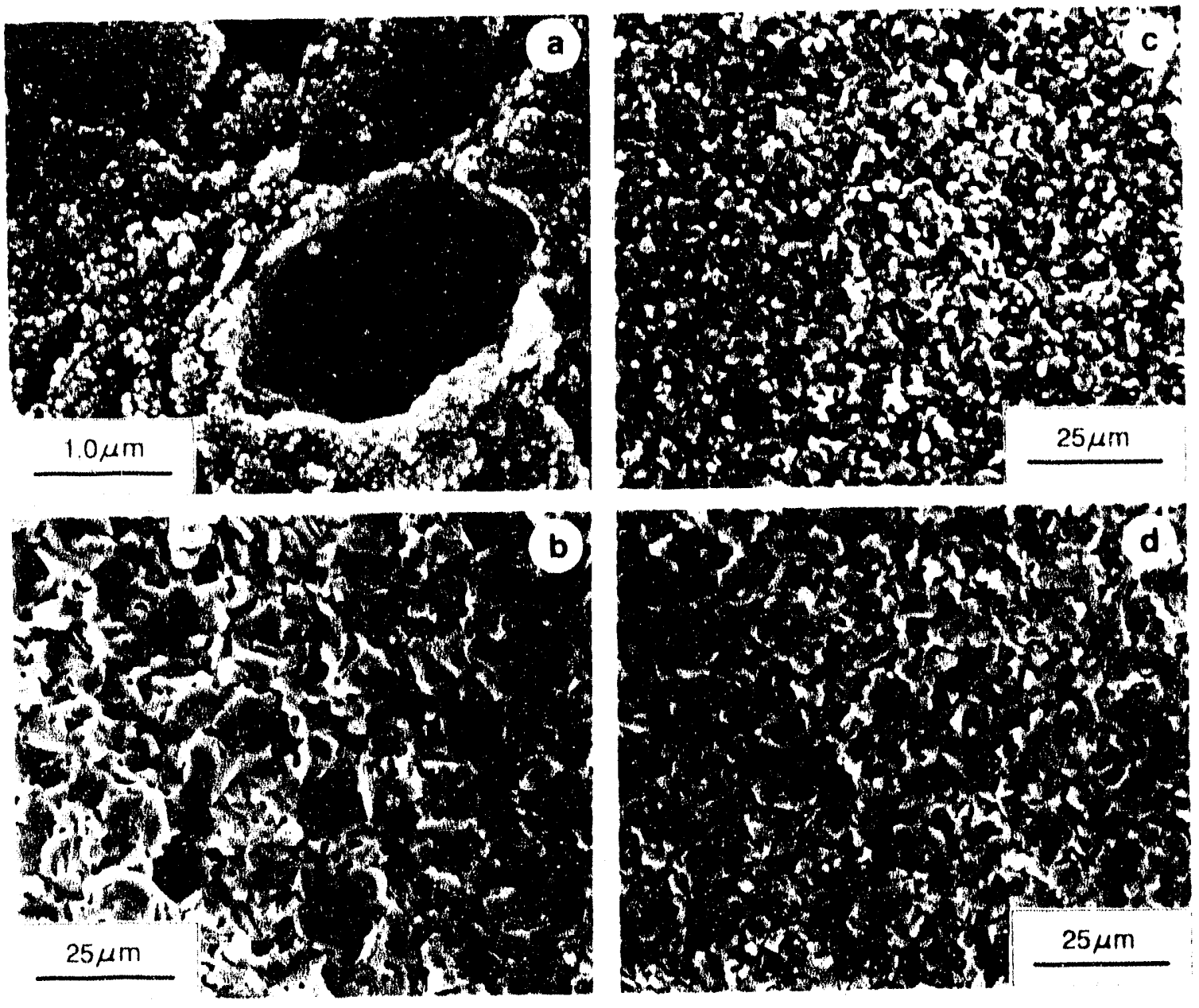

XBB $872.1440 d$

Fig. 5.7 Scanning electron micrographs of fractured surfaces of $\mathrm{MgO}$ compacts. (a) Initial compact microstructure, relative density of 0.40 . (b) Microstructure after isothermal sintering to a relative density of 0.93 . (c) Microstructure after pre-coarsening treatment to a relative density of 0.80 . (d) Microstructure after two-step sintering to a relative density of 0.93 . 

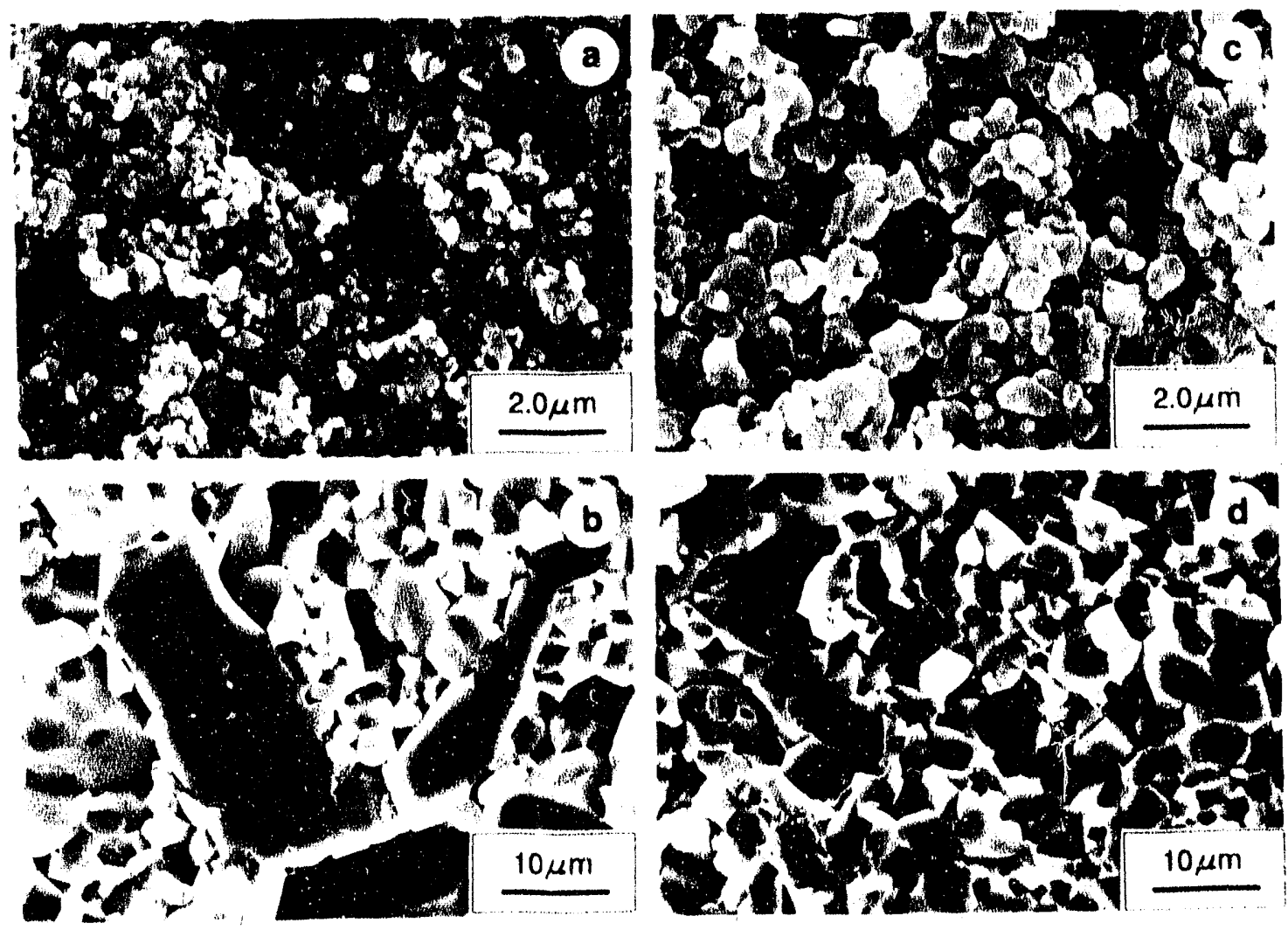

XBB 905-4305a

Fig. 5.8 Scanning electron micrographs of fractured surfaces of $\mathrm{Al}_{2} \mathrm{O}_{3}$ compacts. (a) Initial compact microstructure, relative density of 0.53 . (b) Microstructure after isothermal sintering to a relative density of 0.93 . (c) Microstructure after pre-coarsening treatment to a relative density of 0.83 . (d) Microstructure after two-step sintering to a relative density of 0.95 . 


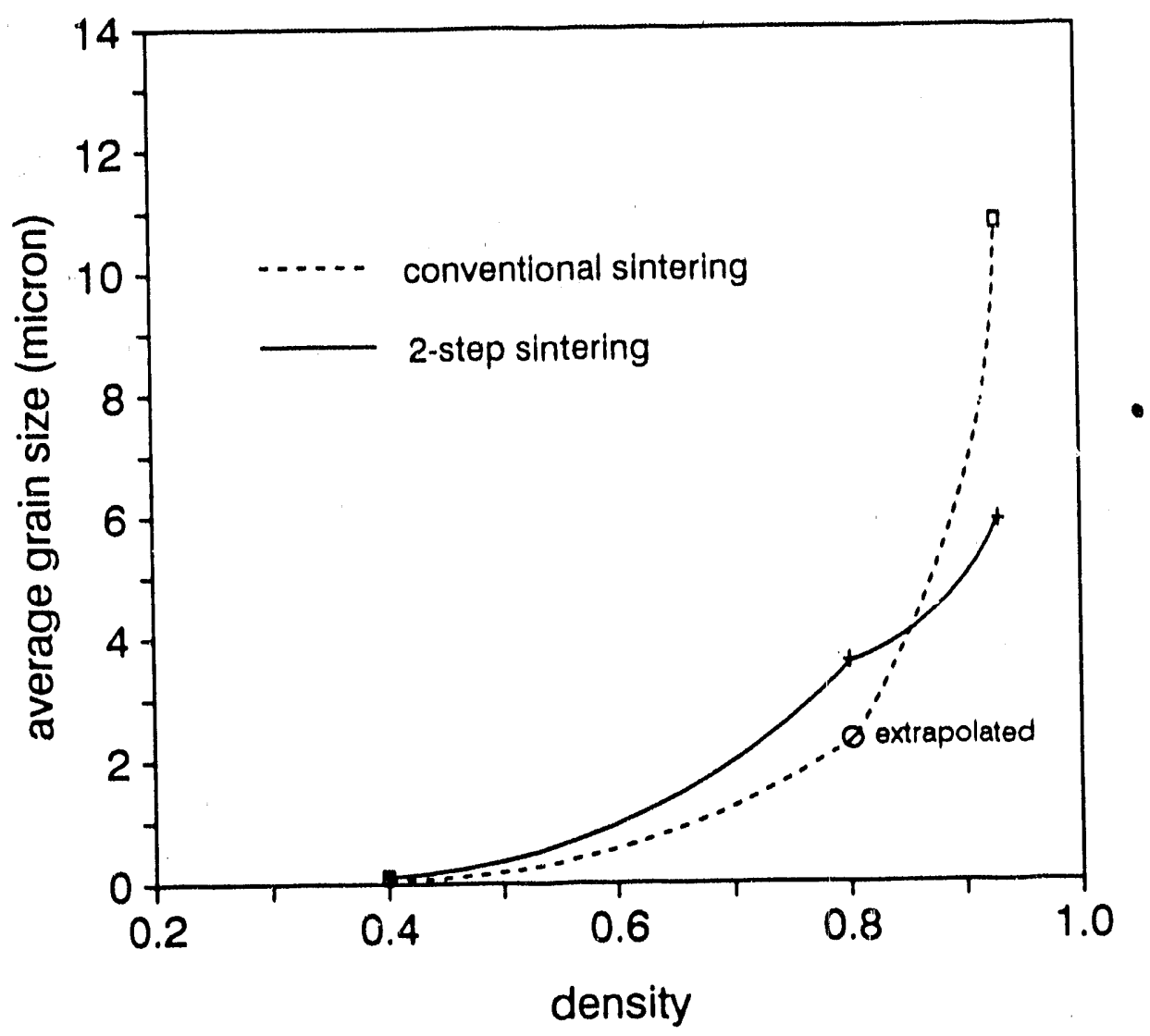

XBL 905-1900

Fig. 5.9 Average grain sizes versus density plot for conventionally sintered and for two-step sintered $\mathrm{MgO}$. 


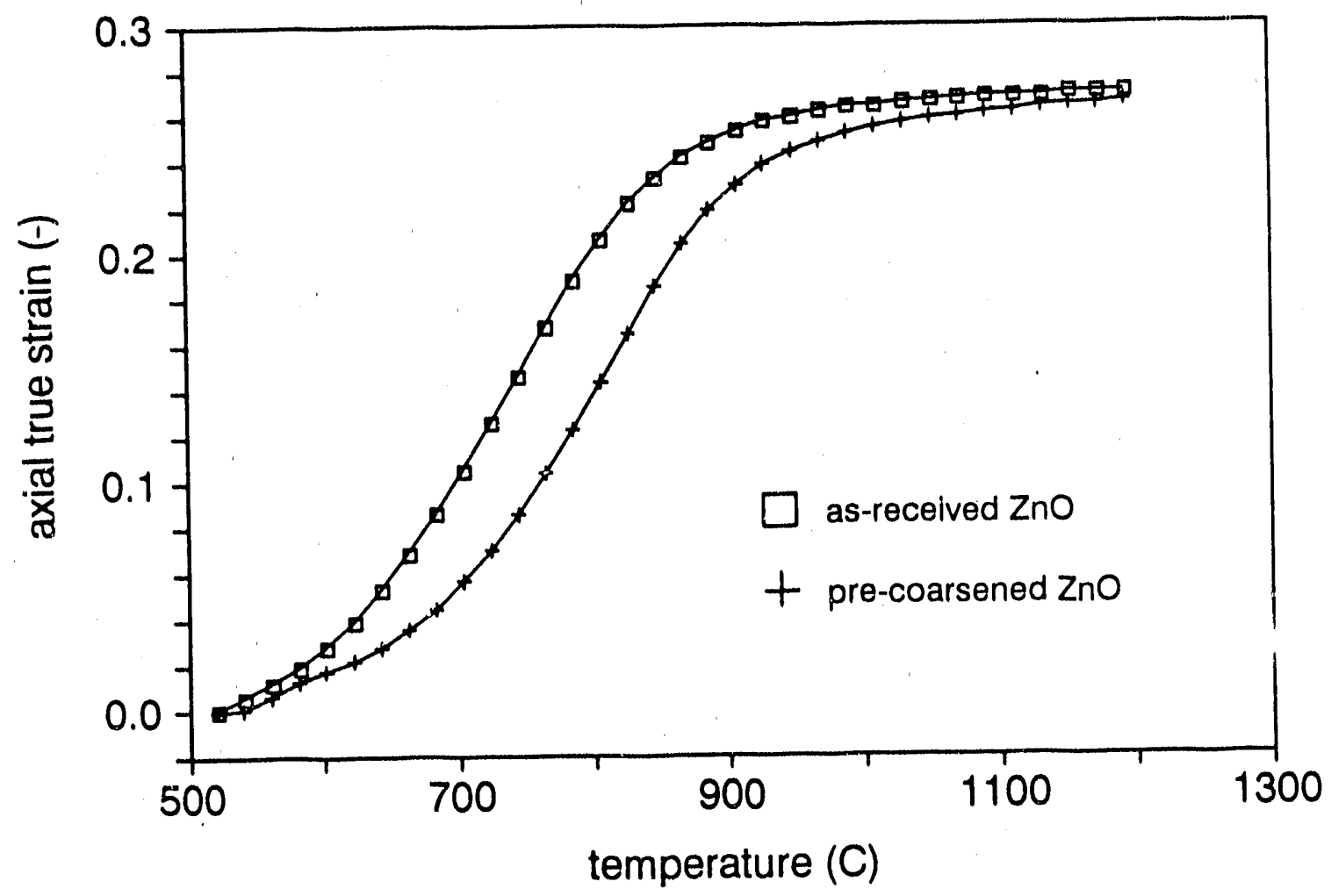

XBL 905-1901

Fig. 5.10 Axial true strain versus temperature for as-received and for pre-coarsened $\mathrm{ZnO}$. 


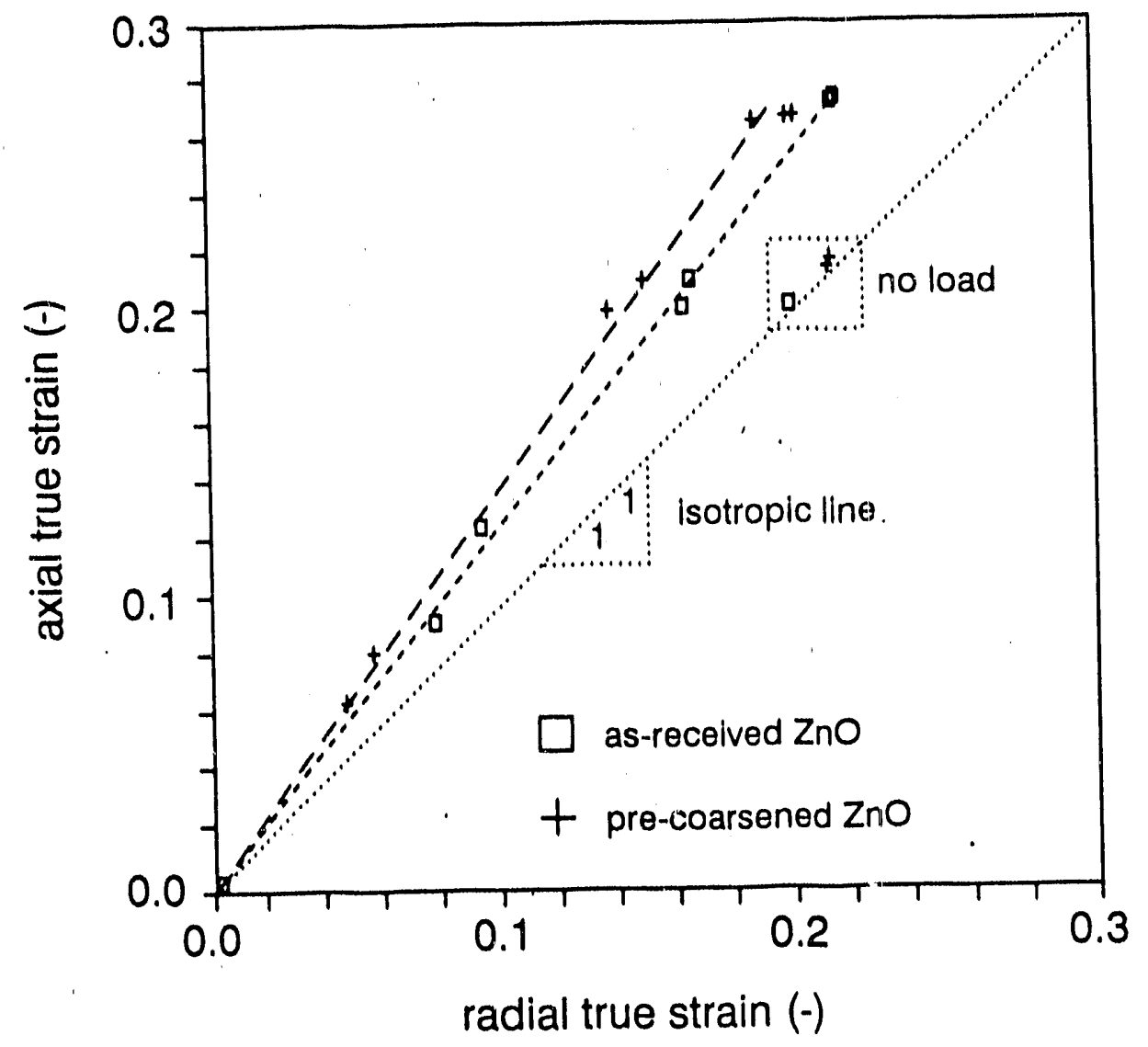

XBL 905-1902

Fig. 5.11 Axial true strain versus radial true strain for as-received and for pre-coarsened $\mathrm{ZnO}$. 


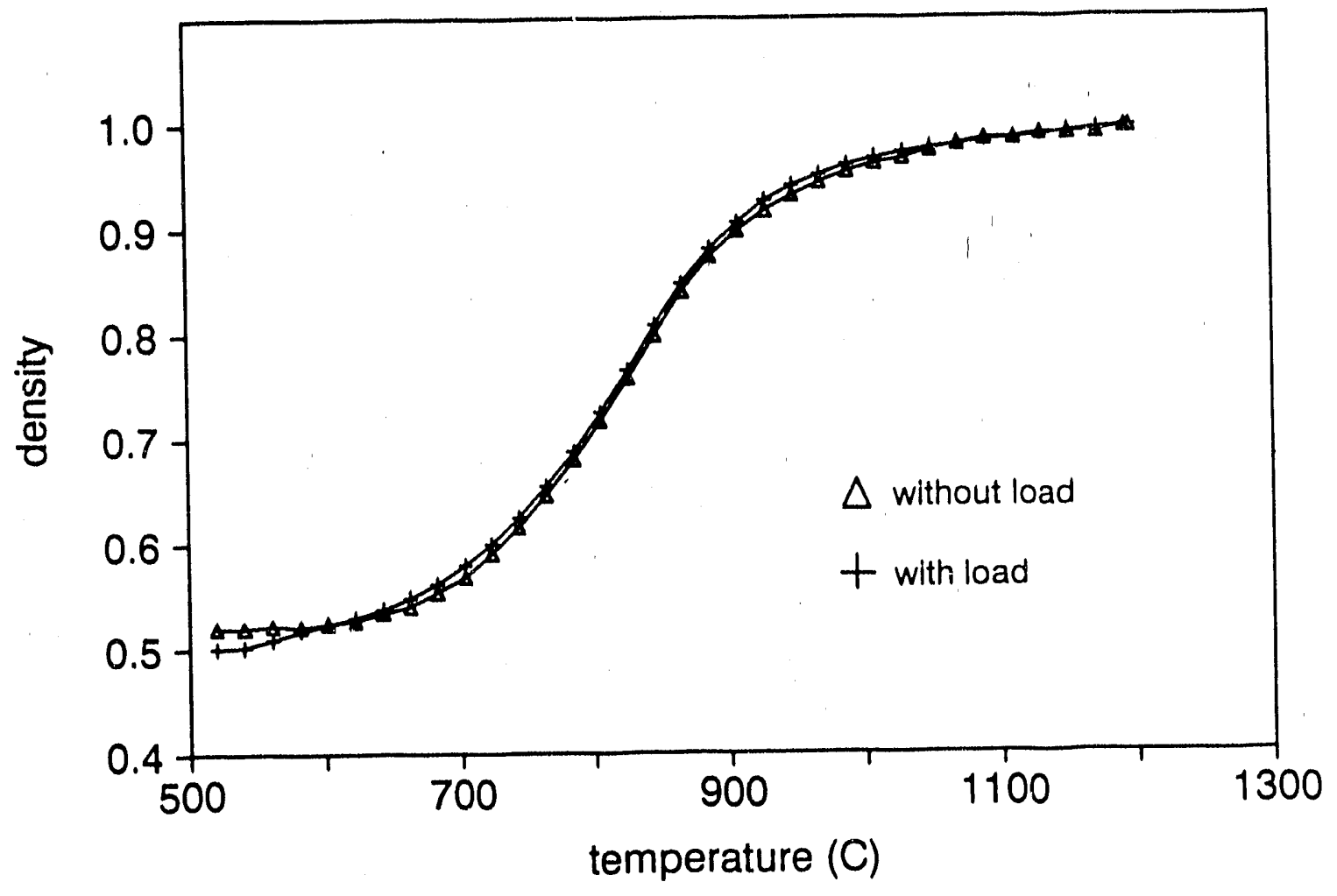

XBL 905-1903

Fig. 5.12 Density versus temperature for pre-coarsened $\mathrm{ZnO}$ sintered without load and sintered with load. 


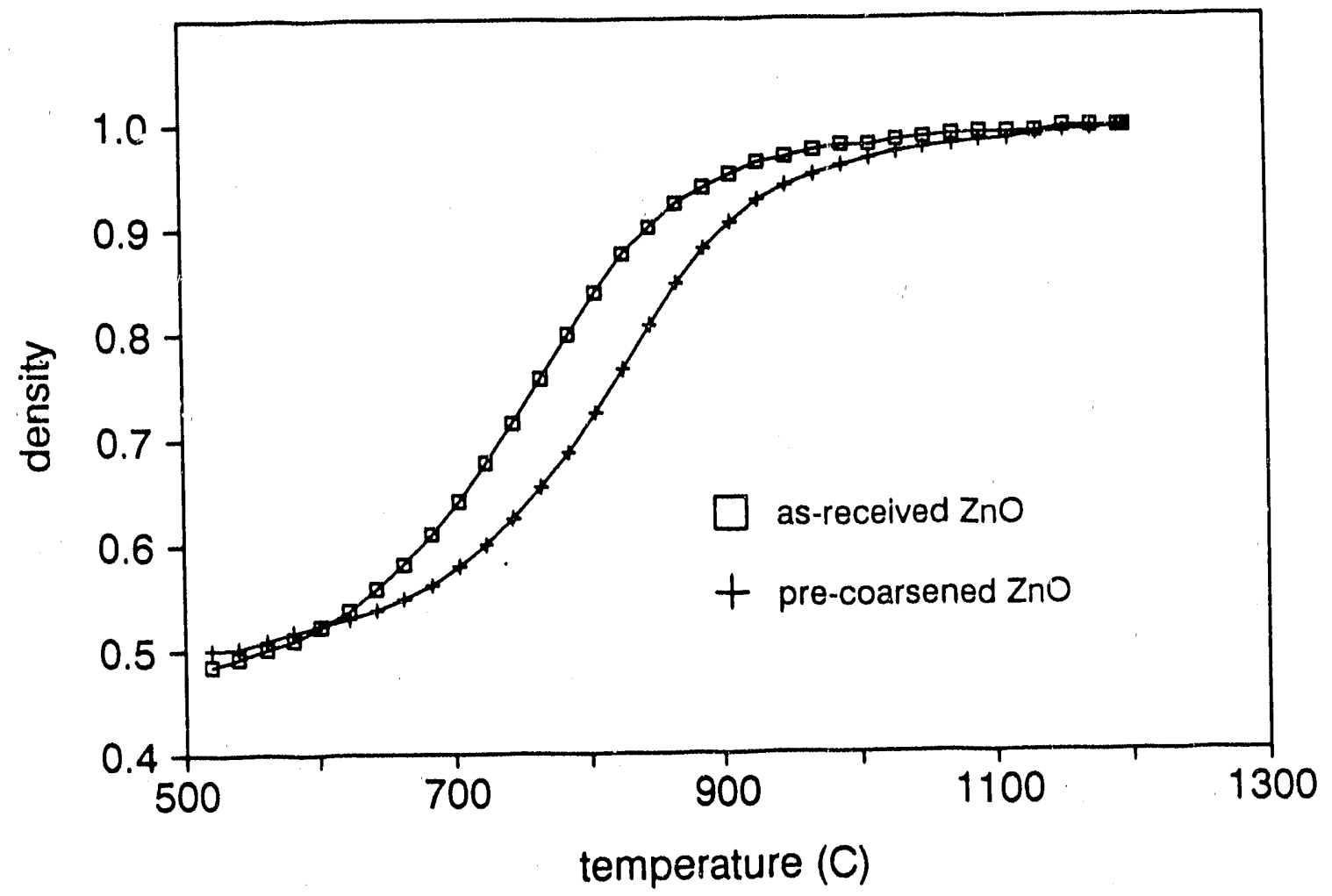

XBL $905-1904$

Fig. 5.13 Density versus temperature for as-received and for pre-coarsened $\mathrm{ZnO}$. 


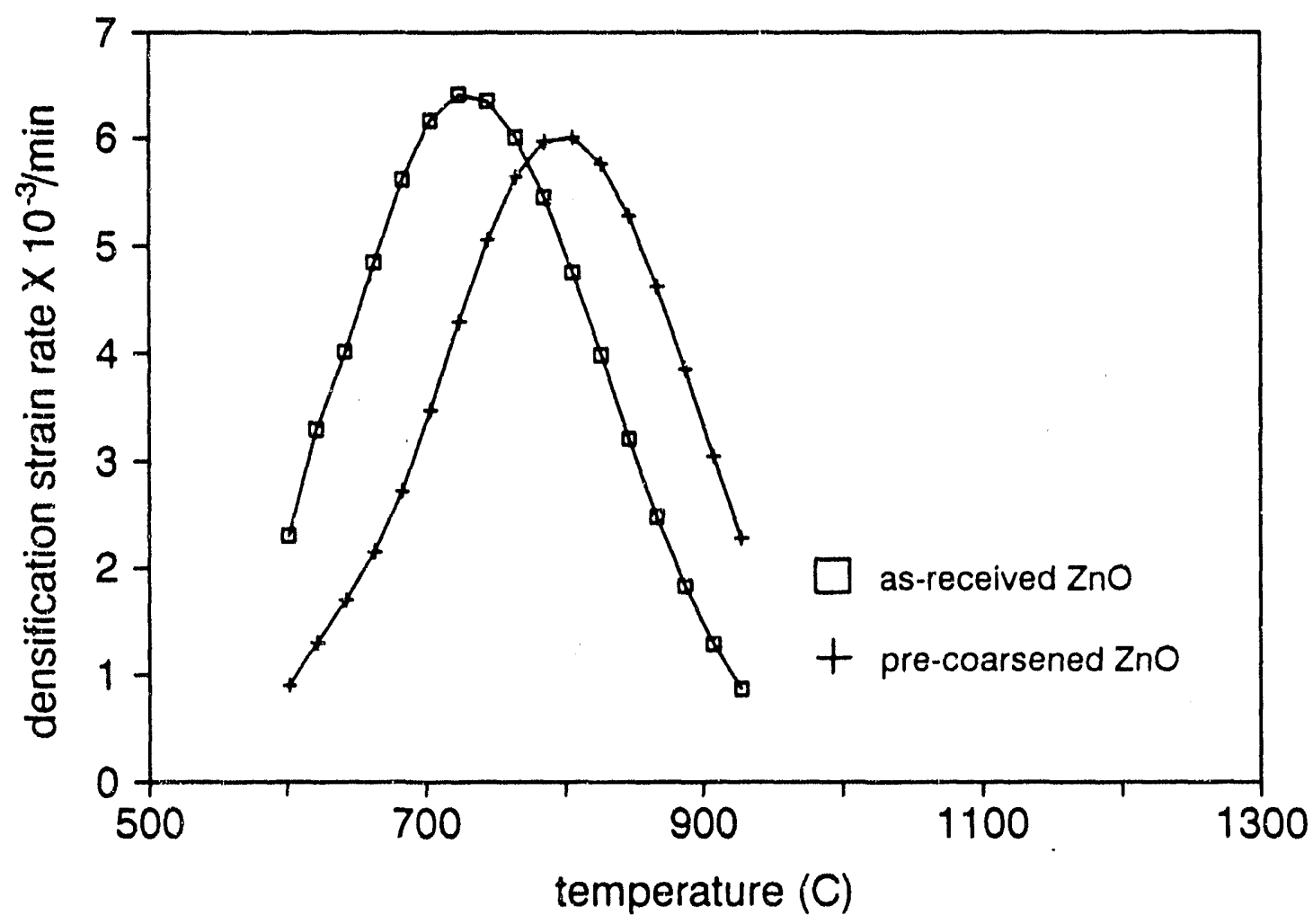

XBL 905-1905

Fig. 5.14 Densification strain rate versus temperature for as-received and for pre-coarsened $\mathrm{ZnO}$. 


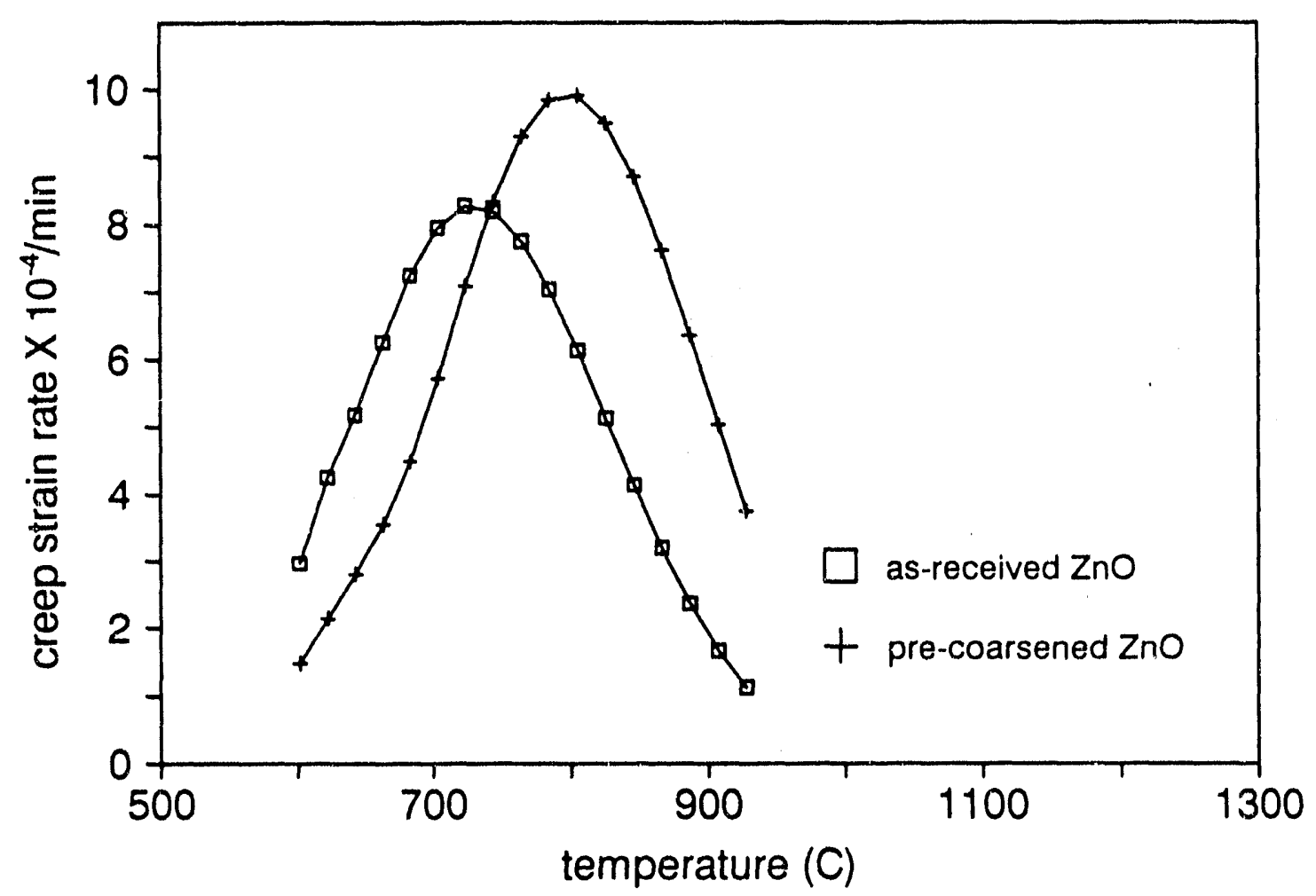

XBL 905-1906

Fig. 5.15 Creep strain rate versus temperature for as-received and for pre-coarsened $\mathrm{ZnO}$. 


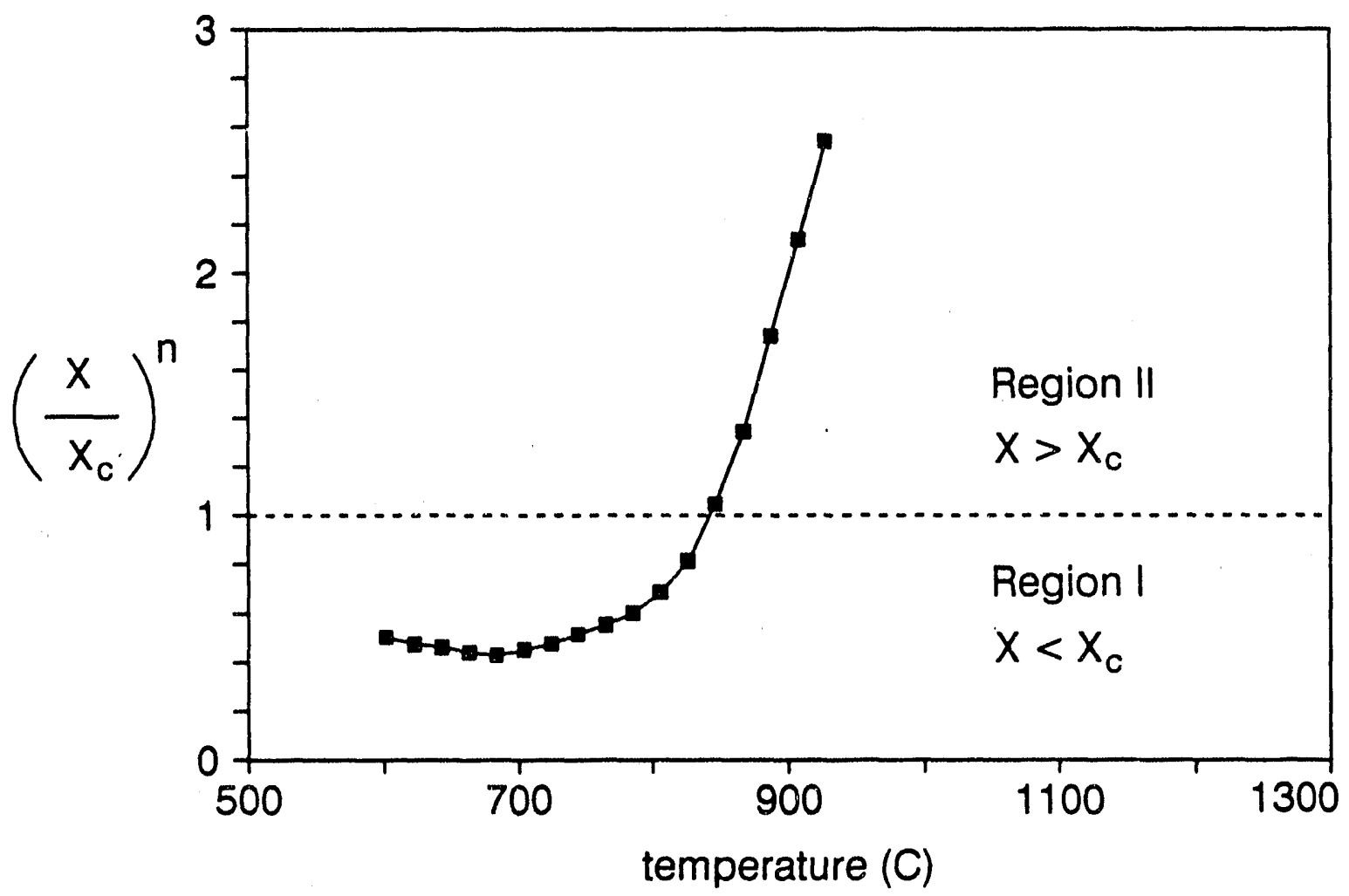

XBL 905-1907

Fig. 5.16 Ratio of the pore spacing of the as-received $\mathrm{ZnO}$, $\mathrm{x}$, over the pore spacing of the pre-coarsened $\mathrm{ZnO}, \mathrm{x}_{\mathrm{C}}$, versus temperature, derived from dilatometry data. 


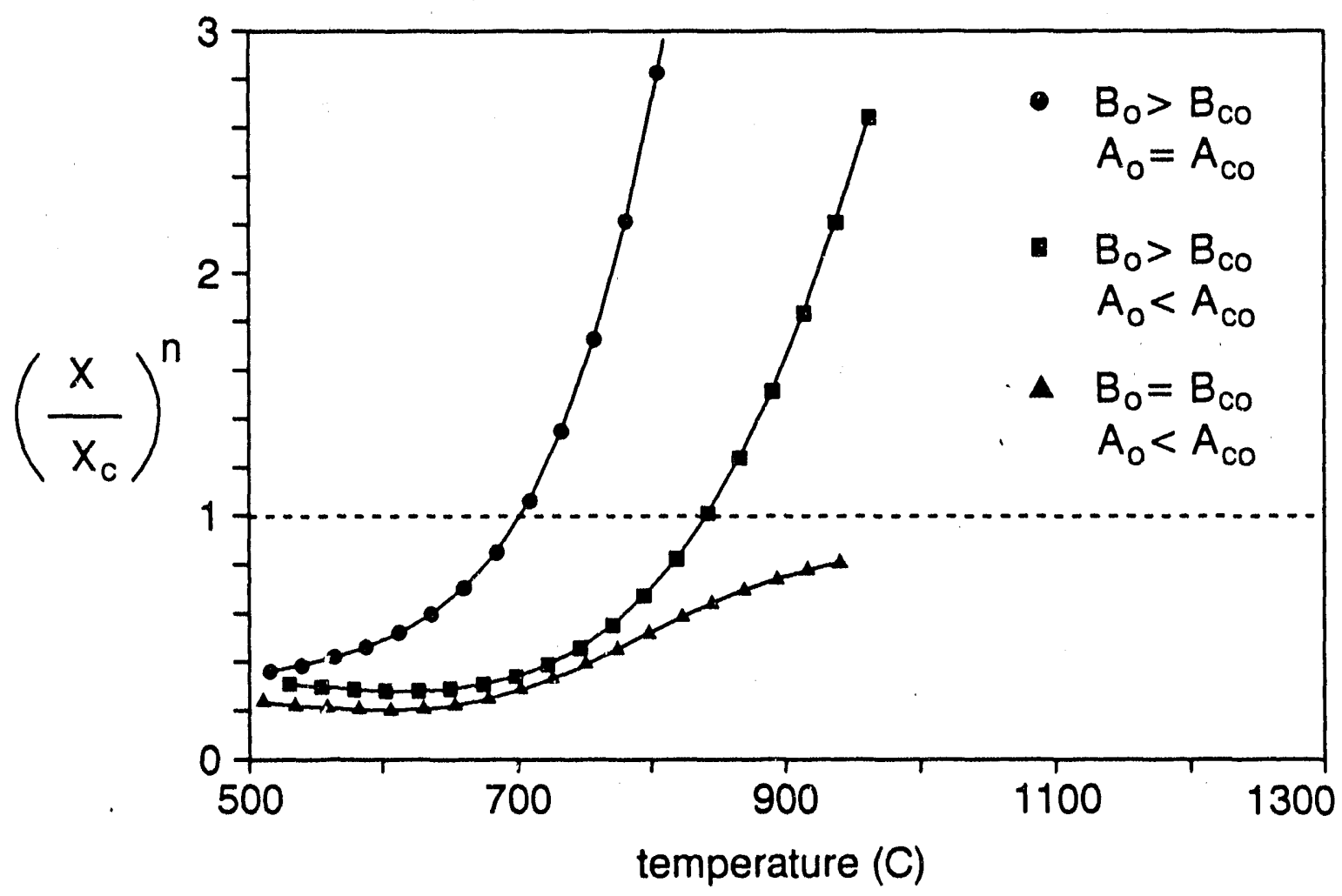

XBL 905-1908

Fig. 5.17 Ratio of the pore spacing of the as-received $\mathrm{ZnO}, \mathrm{x}$, over the pore spacing of the pre-coarsened $\mathrm{ZnO}, \mathrm{x}_{\mathrm{c}}$, versus temperature from numerical calculations with the various indicated choices of parameters. 

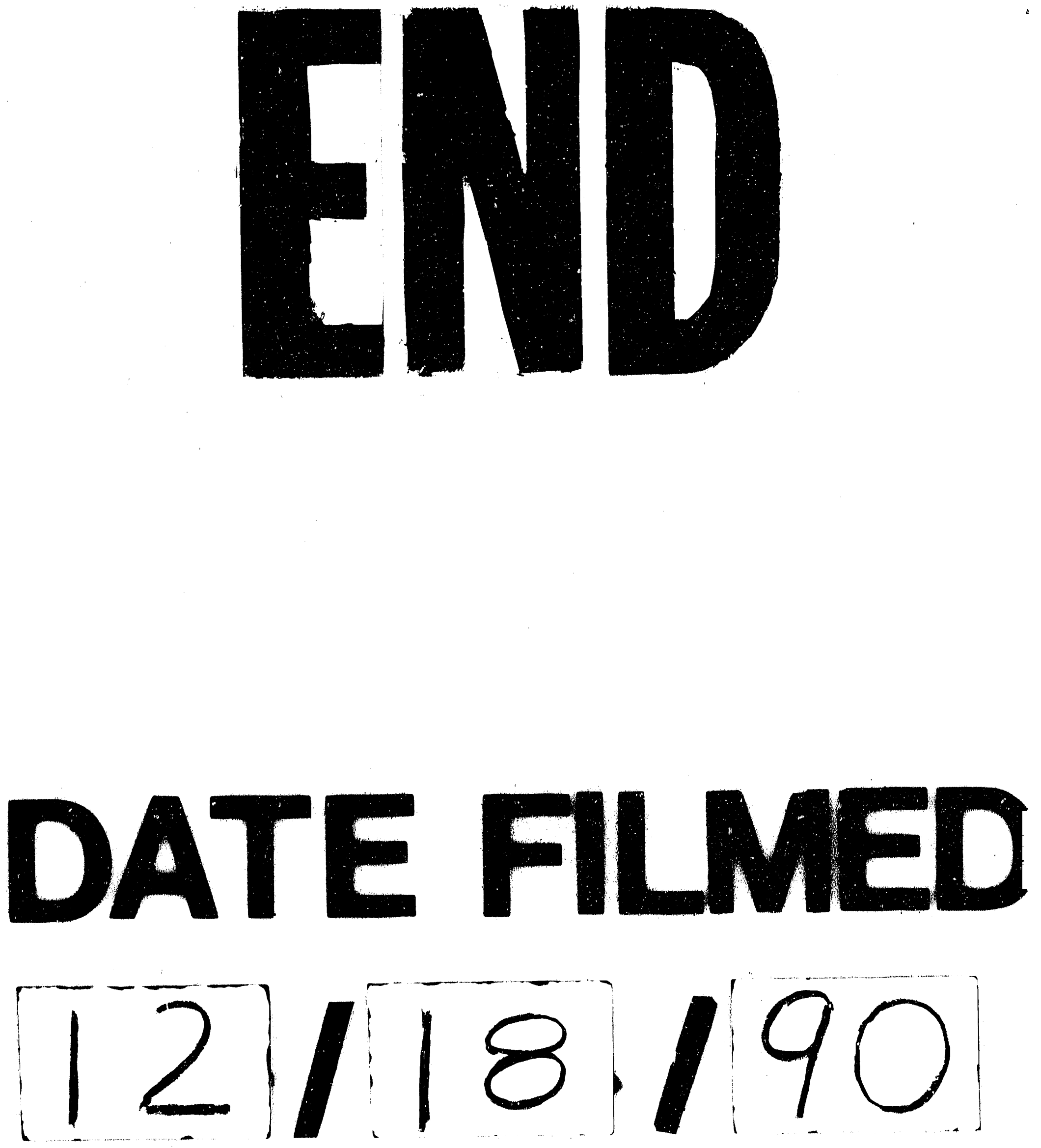
